\title{
Estimation and Testing Using Jackknife IV in Heteroskedastic Regressions With Many Weak Instruments*
}

\author{
John C. Chao ${ }^{1}$ and Norman R. Swanson ${ }^{2}$ \\ ${ }^{1}$ University of Maryland and ${ }^{2}$ Rutgers University \\ March 2004 - preliminary, incomplete and not spell checked!!
}

\begin{abstract}
This paper develops Wald type tests for general possibly nonlinear restrictions, in the context of heteroskedastic IV regression with many weak instruments. In particular, it is first shown that consistency and asymptotically normality can be obtained when estimating structural parameters using JIVE, even when errors exhibit heteroskedasticity of unkown form. This is not the case, however, with other well known IV estimators, such as LIML, Fuller's modified LIML, 2SLS, and B2SLS, which are shown to be inconsistent. Second, new covariance matrix estimators (and corresponding Wald test statistics) are proposed for JIVE, which are consistent even when instrument weakness is such that the rate of growth of the concentration parameter, $r_{n}$, is slower than the rate of growth of the the number of instruments, $K_{n}$, and possibly much slower than the sample size, $n$, provided that $\frac{\sqrt{K_{n}}}{r_{n}} \rightarrow 0$ as $n \rightarrow \infty$. The primary advantage of our tests, relative to those proposed previously in the literature, is that one can test general nonlinear hypotheses, as opposed to simple null hypotheses of the form $H_{0}: \beta=\beta^{*}$, where $\beta^{*}$ is the value of $\beta$ under the null. We feel that this feature, taken together with the fact that the tests are robust to unconditional heteroskedasticity, is important from the perspective of empirical application, given that general linear and nonlinear hypotheses are often of interest to empirical researchers, and given that heteroskedasticity is prevalent, particularly in microeconomic datasets.
\end{abstract}

JEL classification: C13, C31.

Keywords: heteroskedasticity, instrumental variables, Jackknife estimation, local-to-zero framework, many instruments, pathwise asymptotics, weak instruments.

\footnotetext{
* John C. Chao: Department of Economics, University of Maryland, College Park, MD, USA 20742, chao@econ.umd.edu. Norman R. Swanson: Department of Economics, Rutgers University, New Brunswick, NJ, USA 80901, nswanson@econ.rutgers.edu. Earlier versions of this paper were presented at the NSF/NBER conference on weak and/or many instruments at MIT in 2003, and at the 2004 winter meetings of the Econometric Society in San Diego, where conference participants provided many useful comments and suggestions. Particular thanks are owed to Marcelo Moriera, Whitney Newey and James Stock for helpful discussions.
} 


\section{Introduction}

In this paper, we discuss Wald type tests for general possibly nonlinear restrictions, in the context of heteroskedastic IV regression with many weak instruments. The test statistics for which we obtain limiting results are constructed using the jackknife instrumental variables estimator (JIVE), which can be shown to be consistent and asymptotically normal, even when errors exhibit heteroskedasticity of unkown form. These results add to the literature on JIVE estimators that stemmed from a number of important papers including Phillips and Hale (1977), Angrist, Imbens, and Krueger (1999), and Blomquist and Dahlberg (1999). Other well known IV estimators, such as LIML, Fuller's modified LIML, 2SLS, and B2SLS, are shown not to be consistent in our framework, and thus cannot be used when carrying out asymptotically valid tests. An important theoretical result of this paper is the development of new covariance matrix estimators for the two JIVE estimators which are analyzed. Of note is that these covariance matrices are shown to be consistent even when instrument weakness is such that the rate of growth of the concentration parameter, $r_{n}$, is slower than the rate of growth of the number of instruments, $K_{n}$, and possibly much slower than

the sample size, $n$, provided that $\frac{\sqrt{K_{n}}}{r_{n}} \rightarrow 0$ as $n \rightarrow \infty$. The fact that our tests are designed for general possibly nonlinear restrictions, taken together with the fact that the tests are robust to unconditional heteroskedasticity, is important from the perspective of empirical application, given that general linear and nonlinear hypotheses are often of great interest to empirical researchers, and given that heteroskedasticity is prevalent, particularly in microeconomic datasets.

Related recent papers on testing include the those by Kleibergen (2002), Moriera (2003), and Guggenberger and Smith (2003). However, to the best of our knowledge, these papers only address the case of testing simple null hypotheses of the form $H_{0}: \beta=\beta^{*}$, where $\beta^{*}$ is the value of $\beta$ under the null, while our approach allows for straightforward analysis of general coefficient restrictions. In addition, we allow for (unconditional) heteroskedasticity of unkown functional form. Guggenberger and Smith (2003) also address the issue of heteroskedasticity, but only consider conditional forms. However, their setup allows for nonlinear models, while our model is linear. One of the main features accounting for the differences between the approach of the above authors and ours is that they assume that $K_{n}$ is fixed (in the local to zero framework of Staiger and Stock (1997)), and hence consistent estimation of structural parameters cannot be obtained. This precludes testing hypotheses other than those for which all weakly identified structural parameters are known under 
the null. The advantage of their approach is that their tests still have $\chi^{2}$ limiting distributions, even when structural parameters are unidentified with respect to the class of null hypotheses that they consider. Our tests, on the other hand, are designed to handle general hypotheses of the form $H_{0}: h\left(\beta_{0}\right)=0$, where $h(\cdot): \Re^{d} \rightarrow \Re^{q}$, the caveat being that we require at least weak identification, and so does not work if the instrumentrs are completely irrelevant. In particular, in the Staiger and Stock (1997) local to zero framework, Wald test statistics do not have limiting $\chi^{2}$ distributions (the distributions are nonstandard and nuisance parameter dependent). By allowing the number of instruments to increase with the sample size, we are able to obtain consistent estimates of all parameters in our structural equation, hence leading to nuisance parameter free $\chi^{2}$ limiting distributions. Our results can thus be viewed as complimentary to those of Stiager and Stock (1997), in the sense that we consider the case of many weak instruments, while they consider the important case of few weak instruments. In addition, our results can be viewed as adding to the recent literature on many weak instruments (see e.g. Chao and Swanson (2002), Chao and Swanson (2003), Kleibergen (2003), Han and Phillips (2003), and Stock and Yogo (2003).

An ancillary contribution of this paper concerns the development of deeper understanding of how heteroskedasticity affects estimation under many weak instruments. In particular, it is first worth noting that there have been many recent papers which included Monte Carlo experiments exploring the finite sample properties of IV estimators in a weak instrument context. A few of the important papers in this area include Staiger and Stock (1997), Angrist, Imbens, and Krueger (1999), Blomquist and Dahlberg (1999), Gao and Lahiri (2000), Donald and Newey (2001), Hahn, Hausman and Kuersteiner (2002), Hahn and Inoue (2002), and Gugenberger and Smith (2003). Many of these papers involve homoskedastic experimental designs, and many assume that $K_{n}$ is fixed. Exceptions to the former feature include Hahn, Hausman and Kuersteiner (2002) and Guggenberger and Smith (2003), who also include heteroskedastic experiments in their Monte Carlo designs. In particular, their designs take the natural approach of including the heteroskedasticity in the error of the structural equation. Interestingly, a straightforward implication of the findings in this paper and the related findings in Chao and Swanson (2002) is that what causes inconsistency of LIML, Fuller's modified LIML, and B2SLS is heteroskedasticity in the covariance across error terms in the structural and the first stage equations. ${ }^{1}$ Having heteroskedasticy only in the errors of

\footnotetext{
${ }^{1}$ Note that the 2 SLS estimator is inconsistent even under homoskedastic assumptions, in the many weak instruments framework, unless $\frac{K_{n}}{r_{n}} \rightarrow 0$ as $n \rightarrow \infty$ (see Chao and Swanson (2002)).
} 
the structural equation will not preclude the consistency of LIML, Fuller's LIML, and B2SLS. Thus, when experiments are designed in which the objective is to examine the many weak instruments case (i.e. where $K_{n}$ grows), it is also of interest to have experimental designs which assume covariance heteroskedasticity across equation errors.

In the many weak instruments literature, important precedents to our paper include those of Morimune (1983), Bekker (1994), Donald and Newey (2001), Hahn and Hausman (2001), Hahn (2002), Han and Phillips (2003), and Stock and Yogo (2003). Of these, the closest to ours are Stock and Yogo (2003) and Han and Phillips (2003). Stock and Yogo (2003) consider many weak instruments in the case of homoskedastic errors, and consider scenarios where $r_{n}$ grows at the same rate as $K_{n}$. Their results provide important insights into estimation and testing, and establish that standard Wald test statistics have non-pivotal limiting distributions. Han and Phillips (2003) develop a GMM framework, and derive asymptotic distributions of point estimators. Our paper, in summary, adds to previous discussions of many weak instruments by developing tests with nuisance parameter free limiting distributions based on a new covariance matrix estimator that is robust to unconditional heteroskedasticity. In addition, we add to the discussion of IV estimator consistency, by outlining conditions under which various estimators are and are not consistent. For example, our estimation results offer a trade-off relative to those of Han and Phillips (2003). In particular, we are able to obtain consistency and asymptotic normality with weaker instruments, as measured by the rate of growth of the concentration parameter, than in Han and Phillips. The trade-off is that their results apply to a more general possibly nonlinear GMM model, while ours are shown to be valid in an explicitly linear model.

The rest of the paper proceeds as follows. Section 2 sets up the model and discusses our assumptions. Section 3 briefly describes $J I V E$ estimators and presents the main estimation results of the paper. Section 4 discusses testing, and Section 5 contains the results of a small Monte Carlo experiment examning the finite samples properites of our tests. Section 6 concludes. All proofs are gathered in separte appendices on estimation and testing. The following notation is used in the remainder of the paper: $\operatorname{Tr}(\cdot)$ denotes the trace of a matrix, " $>0$ " denotes positive definiteness when applied to matrices, $\varliminf_{n \rightarrow \infty} a_{n}$ denotes the limit inferior of the sequence $\left\{a_{n}\right\}$, and $\varlimsup_{n \rightarrow \infty} a_{n}$ denotes the limit superior of the sequence $\left\{a_{n}\right\}$. In addition, $P_{X}=X\left(X^{\prime} X\right)^{-1} X^{\prime}$ denotes the matrix which projects orthogonally onto the range space of $X$ and $M_{X}=I-P_{X}$. 


\section{$2 \quad$ Model and Assumptions}

Consider the following two-equation simultaneous equations model (SEM)

$$
\begin{aligned}
& y_{1 n}=Y_{2 n} \beta+u_{n}, \\
& Y_{2 n}=Z_{n} \Pi+V_{n},
\end{aligned}
$$

where $y_{1 n}$ and $Y_{2 n}$ are, respectively, an $n \times 1$ vector and an $n \times d$ matrix of observations on the $d+1$ endogenous variables of the system, $Z_{n}$ is an $n \times K_{n}$ matrix of observations on the $K_{n}$ instrumental variables, or exogenous variables excluded from the structural equation (1), and $u_{n}, V_{n}$ are, respectively, an $n \times 1$ vector and an $n \times d$ matrix of random disturbances. Further, let $\xi_{i}=\left(u_{i}, v_{i}^{\prime}\right)^{\prime}$ where $u_{i}$ and $v_{i}^{\prime}$ are, respectively, the $i \underline{t h}$ component of the random vector $u_{n}$ and the $i \underline{t h}$ row of the random matrix $V_{n}$. The following assumptions are used to establish asymptotic normality. ${ }^{2}$

Assumption 1: $\Pi=\Pi_{n}=\frac{C_{n}}{b_{n}}$ for some sequence of positive real numbers $\left\{b_{n}\right\}$, nondecreasing in $n$, and for some sequence of nonrandom, $K_{n} \times d$ parameter matrices $\left\{C_{n}\right\}$.

Assumption 2: Let $\left\{Z_{n, i}: i=1, \ldots, n ; n \geq 1\right\}$ be a triangular array of $R^{K_{n}}$-valued random variables, where $Z_{n, i}^{\prime}$ denotes the $i \underline{t h}$ row of the matrices $Z_{n}$. Also: (a) let $m_{n} \nearrow \infty$ as $n \rightarrow \infty$, and suppose that there exist constants $\underline{D}_{\lambda}$ and $\bar{D}_{\lambda}$, with $0<\underline{D}_{\lambda} \leq \bar{D}_{\lambda}<\infty$, such that $\underline{D}_{\lambda} \leq$ $\varliminf_{n \rightarrow \infty} \lambda_{\min }\left(\frac{Z_{n}^{\prime} Z_{n}}{m_{n}}\right)$ a.s. and $\varlimsup_{n \rightarrow \infty} \lambda_{\max }\left(\frac{Z_{n}^{\prime} Z_{n}}{m_{n}}\right) \leq \bar{D}_{\lambda}$ a.s.; (b) assume that $K_{n} \rightarrow \infty$ as $n \rightarrow \infty$ such that $\frac{K_{n}^{2} n}{m_{n}^{2}} \rightarrow 0$; and (c) assume that there exist constants $\underline{D}_{C}$ and $\bar{D}_{C}$, with $0<\underline{D}_{C} \leq \bar{D}_{C}<\infty$, such that $\underline{D}_{C} \leq \varliminf_{n \rightarrow \infty} \lambda_{\min }\left(\frac{C_{n}^{\prime} C_{n}}{K_{n}}\right)$ and $\varlimsup_{n \rightarrow \infty} \lambda_{\max }\left(\frac{C_{n}^{\prime} C_{n}}{K_{n}}\right) \leq \bar{D}_{C}$.

Assumption 3: (a) Define $\Sigma_{i}\left(Z_{n}\right)=E\left(\xi_{i} \xi_{i}^{\prime} \mid Z_{n}\right)$ and suppose that $\xi_{i} \mid Z_{n} \equiv$ i.n.i.d. $\left(0, \Sigma_{i}\left(Z_{n}\right)\right)$ almost surely for all $n$, where $\xi_{i} \mid Z_{n}$ denotes the conditional distribution of $\xi_{i}$ given $Z_{n}$. Further, assume that $\Sigma_{i}\left(Z_{n}\right)>0$ for all $i$ and $n$ almost surely, and partition $\Sigma_{i}\left(Z_{n}\right)$ conformably with $\xi_{i}=\left(u_{i}, v_{i}^{\prime}\right)^{\prime}$ as $\Sigma_{i}\left(Z_{n}\right)=\left(\begin{array}{cc}\sigma_{u u, i}\left(Z_{n}\right) & \sigma_{V u, i}^{\prime}\left(Z_{n}\right) \\ \sigma_{V u, i}\left(Z_{n}\right) & \Sigma_{V V, i}\left(Z_{n}\right)\end{array}\right)$; (b) assume that there exists some constant $\bar{D}_{\xi}$, with $0<\bar{D}_{\xi}<\infty$, such that $\max \left\{\sup _{i} E\left(u_{i}^{4} \mid Z_{n}\right), \sup _{i} E\left(v_{i 1}^{4} \mid Z_{n}\right), \ldots, \sup _{i} E\left(v_{i d}^{4} \mid Z_{n}\right)\right\} \leq \bar{D}_{\xi}$ almost surely; and (c) assume that there exists some constant $\underline{D}_{\xi}$, with $0<\underline{D}_{\xi}<\infty$, such that $\min \left\{\inf _{i} \sigma_{u u, i}\left(Z_{n}\right), \inf _{i} \lambda_{\min }\left(\Sigma_{V V, i}\left(Z_{n}\right)\right)\right\} \geq \underline{D}_{\xi}$ almost surely.

Assumption 4: Assume that there exists a constant $D_{Z}$, with $0<D_{Z}<\infty$, such that

\footnotetext{
${ }^{2}$ Stronger versions of Assumptions 2-4 are later made in order to obtain asymptotics for Wald tests.
} 
$\sup _{1 \leq i \leq n, \leq k \leq K_{n}, n \geq 1} E\left(Z_{i k, n}^{4}\right) \leq D_{Z}$, where $Z_{i k, n}$ denotes the $(i, k)^{t h}$ element of $Z_{n}$.

Assumption 5: Define the ratio $r_{n}=\frac{m_{n} K_{n}}{b_{n}^{2}}$. Suppose that, as $n \rightarrow \infty, \frac{r_{n}}{n} \rightarrow \kappa$ for some constant $\kappa$ such that $0 \leq \kappa<\infty$.

Assumptions 1 and 2 are similar to corresponding assumptions that were made in Chao and Swanson $(2002,2003)$. As explained in those papers, these assumptions imply that there exists a positive integer $N$ such that, for all $n \geq N, 0<\underline{D}_{\lambda} \underline{D}_{C} \leq \frac{\Pi_{n}^{\prime} Z_{n}^{\prime} Z_{n} \Pi_{n}}{r_{n}} \leq \bar{D}_{\lambda} \bar{D}_{C}<\infty$ with probability one, so that the concentration parameter $\Pi_{n}^{\prime} Z_{n}^{\prime} Z_{n} \Pi_{n}$ grows at the rate $r_{n}=\frac{m_{n} K_{n}}{b_{n}^{2}}$ under these conditions. It is sensible to have conditions which characterize the rate of growth of the concentration parameter since the concentration parameter is a natural measure of instrumental strength, as has been pointed out by numerous authors, including Phillips (1983), Rothenberg (1983), and Stock and Yogo (2003). Because we are interested in the case of weak instruments, Assumption 5 stipulates that $r_{n}$ must grow no faster than $n$. In fact, we will be interested primarily in the case where $r_{n}$ grows much more slowly than $n$. Note also that $m_{n}$ in Assumption 2(a) represents the rate of growth of the matrix $Z_{n}^{\prime} Z_{n}$ or the rate at which information in the instruments accumulates. On the other hand, Assumption 2(b) places a restriction on the growth rate of the number of instruments as $n \rightarrow \infty$. In the typical case where $m_{n}=n$, Assumption 2(b) reduces to $\frac{K_{n}^{2}}{n} \rightarrow 0$ as $n \rightarrow \infty$, which is the same as a condition assumed in Stock and Yogo (2003).

Finally, note that Assumption 3(a) allows for heteroskedasticity of unknown form in the errors of the model. In this sense, our setup generalizes the homoskedastic, many-instruments framework used by many previous authors, including Morimune (1983), Bekker (1994), Angrist and Krueger (1995), Donald and Newey (2001), Chao and Swanson (2002, 2003), and Stock and Yogo (2003). Our assumption on the stochastic properties of the instruments are also weaker than what has previously been assumed in the many-instruments literature, as previous authors have either assumed fixed (non-random) instruments or stochastic i.i.d. instruments, whereas our assumptions allow for stochastic instruments which are not necessarily i.i.d.

\section{Asymptotic Normality}

Consider two alternative versions of JIVE due to Phillips and Hale (1977) and Angrist, Imbens, and Krueger (1999). Namely, consider 


$$
\begin{aligned}
\widehat{\beta}_{J I V E 1, n} & =\left(Y_{2 n}^{\prime}\left[I_{n}-M_{Z_{n}} H_{1 n}\right] Y_{2 n}\right)^{+} Y_{2 n}^{\prime}\left[I_{n}-M_{Z_{n}} H_{1 n}\right] y_{1 n} \\
& =\left(Y_{2 n}^{\prime}\left[P_{Z_{n}} H_{1 n}-H_{2 n}\right] Y_{2 n}\right)^{+} Y_{2 n}^{\prime}\left[P_{Z_{n}} H_{1 n}-H_{2 n}\right] y_{1 n},
\end{aligned}
$$

where $H_{1 n}=\operatorname{diag}\left(\frac{1}{1-p_{11, n}^{Z}}, \frac{1}{1-p_{22, n}^{Z}}, \ldots \ldots ., \frac{1}{1-p_{n n, n}^{Z}}\right)$ and $H_{2 n}=\operatorname{diag}\left(\frac{p_{11, n}^{Z}}{1-p_{11, n}^{Z}}, \frac{p_{22, n}^{Z}}{1-p_{22, n}^{Z}}, \ldots \ldots ., \frac{p_{n n, n}^{Z}}{1-p_{n n, n}^{Z}}\right)$ and where $p_{i i, n}^{Z}$ denotes the $i^{\text {th }}$ diagonal element of the projection matrix $P_{Z_{n}}$. As explained in Angrist, Imbens, and Krueger (1999) and also in Blomquist and Dahlberg (1999), JIVE1 is an IV estimator constructed from the following two-step procedure. First, construct jackknife fitted values $\widehat{Y}_{2 n, i}^{J I V E 1}, i=1, \ldots, n$, by running first stage OLS regressions using all but the $i$ th observation (i.e. set $\widehat{Y}_{2 n, i}^{J I V E 1}=\widehat{\Pi}_{n}(i)^{\prime} Z_{n, i}$, for $i=1, \ldots, n$, where $\widehat{\Pi}_{n}(i)=\left(Z_{n}(i)^{\prime} Z_{n}(i)\right)^{-1}\left(Z_{n}(i)^{\prime} Y_{2 n}(i)\right)$ is the $O L S$ estimator of the coefficient matrices of equation (2), obtained by deleting the $i$ th observations, and where $Z_{n}(i)$ denotes a submatrix of $Z_{n}$ obtained by deleting the $i^{\text {th }}$ row from the latter). Next, estimate the structural equation (1) by an $I V$ procedure, using the matrix of instruments $\widehat{Y}_{2 n}^{J I V E 1}=\left(\widehat{Y}_{2 n, 1}^{J I V E 1}, \widehat{Y}_{2 n, 2}^{J I V E 1}, \ldots \ldots, \widehat{Y}_{2 n, n}^{J I V E 1}\right)^{\prime}$. Also, consider

$$
\widehat{\beta}_{J I V E 2, n}=\left(Y_{2 n}^{\prime}\left[P_{Z_{n}}-H_{3 n}\right] Y_{2 n}\right)^{+} Y_{2 n}^{\prime}\left[P_{Z_{n}}-H_{3 n}\right] y_{1 n},
$$

where $H_{3 n}=\operatorname{diag}\left(p_{11, n}^{Z}, p_{22, n}^{Z}, \ldots \ldots ., p_{n n, n}^{Z}\right)$. This version of $J I V E$ was first proposed by Angrist, Imbens, and Krueger (1999), who obtained this estimator by modifying step (i) above so that the alternative fitted values $\widehat{Y}_{2 n, i}^{J I V E 2}=\widetilde{\Pi}_{n}(i)^{\prime} Z_{n, i}$, where $\widetilde{\Pi}_{n}(i)=\left(Z_{n}{ }^{\prime} Z_{n}\right)^{-1}\left(Z_{n}(i)^{\prime} Y_{2 n}(i)\right)$, are used in lieu of $\widehat{Y}_{2 n, i}^{J I V E 1}$ for $i=1, \ldots, n$.

For these estimators to be well-defined, we need to make the following additional assumption. Assumption 6: There exists a constant $\bar{h}$, with $0<\bar{h}<1$, such that $0 \leq p_{i i, n}^{Z} \leq \bar{h}$ a.s. for $1 \leq i \leq n$ and for all $n$ sufficiently large such that $P_{Z_{n}}$ is well-defined almost surely. ${ }^{3}$

As explained in Angrist, Imbens, and Krueger (1999), the motivation behind JIVE1 and $J I V E 2$ is to remove, or at least to reduce, the finite sample dependence between the first-stage fitted values and the disturbance of the structural equation by constructing these fitted values

\footnotetext{
${ }^{3}$ Note that Assumption 6 does rule out exogenous regressors of the form $e_{i}=(0, \ldots, 0,1,0, \ldots, 0)$, where $e_{i}$ denotes the $i^{\text {th }}$ elementary vector, or, alternatively, the $i^{\text {th }}$ column of an identity matrix. It is easy to show that $h_{i, n}=1$ if $e_{i}$ is a column of $Z_{n}$. Other types of dummy variables are not ruled out, however. Note also that Assumption 2(b) implies that, for $n$ sufficiently large, the matrix $Z_{n}^{\prime} Z_{n}$ is positive definite almost surely, so that $P_{Z_{n}}$ is well-defined almost surely for large enough $n$.
} 
via "delete one" estimation procedures. This results in a built-in bias correction, which, in turn, enables these estimators to have good asymptotic properties in the sense that, under fairly general conditions, they are consistent and asymptotically normal even in the presence of many weak instruments and heteroskedasticity, as are shown in the following theorems:

Theorem 3.1 (JIVE1): Under Assumptions 1-6, let $\widehat{\beta}_{J I V E 1, n}$ be as defined in equation (3) above. Then: (a) suppose that $\frac{K_{n}}{r_{n}} \rightarrow 0$ as $n \rightarrow \infty$; then,

$$
\left(\Psi_{n}^{-1} \Xi_{1 n} \Psi_{n}^{-1}\right)^{-\frac{1}{2}}\left(\widehat{\beta}_{J I V E 1, n}-\beta_{0}\right) \stackrel{d}{\rightarrow} N\left(0, I_{d}\right) \quad \text { as } n \rightarrow \infty,
$$

where $\Psi_{n}=b_{n}^{-2} C_{n}^{\prime} Z_{n}^{\prime} Z_{n} C_{n}$ and $\Xi_{1 n}=\sum_{j=1}^{n} b_{n}^{-2} C_{n}^{\prime} Z_{n, j} Z_{n, j}^{\prime} C_{n} \sigma_{u u, j}\left(Z_{n}\right) ;\left(\right.$ b) suppose that $\frac{K_{n}}{r_{n}} \rightarrow \delta$, for $0<\delta<\infty$, as $n \rightarrow \infty$; then,

$$
\left(\Psi_{n}^{-1} \Xi_{n} \Psi_{n}^{-1}\right)^{-\frac{1}{2}}\left(\widehat{\beta}_{J I V E 1, n}-\beta_{0}\right) \stackrel{d}{\rightarrow} N\left(0, I_{d}\right) \quad \text { as } n \rightarrow \infty
$$

where $\Xi_{n}=\Xi_{1 n}+\Xi_{2 n}, \Xi_{1 n}$ is as defined above,

$$
\begin{aligned}
\Xi_{2 n}= & \sum_{1 \leq i<j \leq n}\left[g_{i j, n}^{2} \Sigma_{V V, i}\left(Z_{n}\right) \sigma_{u u, j}\left(Z_{n}\right)+g_{i j, n} g_{j i, n} \sigma_{V u, i}\left(Z_{n}\right) \sigma_{V u, j}^{\prime}\left(Z_{n}\right)\right. \\
& \left.+g_{i j, n} g_{j i, n} \sigma_{V u, j}\left(Z_{n}\right) \sigma_{V u, i}^{\prime}\left(Z_{n}\right)+g_{j i, n}^{2} \Sigma_{V V, j}\left(Z_{n}\right) \sigma_{u u, i}\left(Z_{n}\right)\right]
\end{aligned}
$$

and $g_{i j, n}$ denotes the $(i, j)^{t h}$ element of the matrix $G_{n}=P_{Z_{n}} H_{1 n}-H_{2 n}$; and (c) suppose that $\frac{r_{n}}{K_{n}} \rightarrow 0$ and $\frac{\sqrt{K_{n}}}{r_{n}} \rightarrow 0$, as $n \rightarrow \infty$; then,

$$
\left(\Psi_{n}^{-1} \Xi_{2 n} \Psi_{n}^{-1}\right)^{-\frac{1}{2}}\left(\widehat{\beta}_{J I V E 1, n}-\beta_{0}\right) \stackrel{d}{\rightarrow} N\left(0, I_{d}\right) \quad \text { as } n \rightarrow \infty
$$

where $\Psi_{n}=b_{n}^{-2} C_{n}^{\prime} Z_{n}^{\prime} Z_{n} C_{n}$ and $\Xi_{2 n}$ is as defined above.

Theorem 3.2 (JIVE2): Under Assumptions 1-6, let $\widehat{\beta}_{J I V E 2, n}$ be as defined in equation (4) above. Then: (a) suppose that $\frac{K_{n}}{r_{n}} \rightarrow 0$, as $n \rightarrow \infty$; then,

$$
\left(\Psi_{n}^{*-1} \Xi_{1 n}^{*} \Psi_{n}^{*-1}\right)^{-\frac{1}{2}}\left(\widehat{\beta}_{J I V E 2, n}-\beta_{0}\right) \stackrel{d}{\rightarrow} N\left(0, I_{d}\right) \quad \text { as } n \rightarrow \infty
$$

where $\Psi_{n}^{*}=b_{n}^{-2} C_{n}^{\prime} Z_{n}^{\prime}\left[I_{n}-H_{3 n}\right] Z_{n} C_{n}$ and $\Xi_{1 n}^{*}=\sum_{j=1}^{n} b_{n}^{-2}\left(1-p_{j j, n}^{Z}\right)^{2} C_{n}^{\prime} Z_{n, j} Z_{n, j}^{\prime} C_{n} \sigma_{u u, j}\left(Z_{n}\right)$; (b) suppose that $\frac{K_{n}}{r_{n}} \rightarrow \delta$, for $0<\delta<\infty$, as $n \rightarrow \infty$; then,

$$
\left(\Psi_{n}^{*-1} \Xi_{n}^{*} \Psi_{n}^{*-1}\right)^{-\frac{1}{2}}\left(\widehat{\beta}_{J I V E 2, n}-\beta_{0}\right) \stackrel{d}{\rightarrow} N\left(0, I_{d}\right) \quad \text { as } n \rightarrow \infty
$$


where $\Xi_{n}^{*}=\Xi_{1 n}^{*}+\Xi_{2 n}^{*}, \Xi_{1 n}^{*}$ is as defined above,

$$
\begin{aligned}
\Xi_{2 n}^{*}= & \sum_{1 \leq i<j \leq n}\left[\left(g_{i j, n}^{*}\right)^{2} \Sigma_{V V, i}\left(Z_{n}\right) \sigma_{u u, j}\left(Z_{n}\right)+g_{i j, n}^{*} g_{j i, n}^{*} \sigma_{V u, i}\left(Z_{n}\right) \sigma_{V u, j}^{\prime}\left(Z_{n}\right)\right. \\
& \left.+g_{i j, n}^{*} g_{j i, n}^{*} \sigma_{V u, j}\left(Z_{n}\right) \sigma_{V u, i}^{\prime}\left(Z_{n}\right)+\left(g_{j i, n}^{*}\right)^{2} \Sigma_{V V, j}\left(Z_{n}\right) \sigma_{u u, i}\left(Z_{n}\right)\right]
\end{aligned}
$$

and $g_{i j, n}^{*}$ denotes the $(i, j)^{t h}$ element of the matrix $G_{n}^{*}=P_{Z_{n}}-H_{3 n}$; and (c) suppose that $\frac{r_{n}}{K_{n}} \rightarrow 0$ and $\frac{\sqrt{K_{n}}}{r_{n}} \rightarrow 0$, as $n \rightarrow \infty$; then,

$$
\left(\Psi_{n}^{*-1} \Xi_{2 n}^{*} \Psi_{n}^{*-1}\right)^{-\frac{1}{2}}\left(\widehat{\beta}_{J I V E 2, n}-\beta_{0}\right) \stackrel{d}{\rightarrow} N\left(0, I_{d}\right) \quad \text { as } n \rightarrow \infty,
$$

where $\Psi_{n}^{*}=b_{n}^{-2} C_{n}^{\prime} Z_{n}^{\prime}\left[I_{n}-H_{3 n}\right] Z_{n} C_{n}$ and where $\Xi_{2 n}^{*}$ is as defined above.

Suppose we make the additional assumptions:

Assumption 7: As $n \rightarrow \infty, \bar{\Psi}_{n}=r_{n}^{-1} \Psi_{n} \stackrel{\text { a.s. }}{\rightarrow} \bar{\Psi}, r_{n}^{-1} \Xi_{1 n} \rightarrow \bar{\Xi}_{1}$, and $K_{n}^{-1} \Xi_{2 n} \rightarrow \bar{\Xi}_{2}$ for positive definite matrices $\bar{\Psi}, \bar{\Xi}_{1}$, and $\bar{\Xi}_{2}$.

Assumption $7^{\prime}$ : As $n \rightarrow \infty, \bar{\Psi}_{n}^{*}=r_{n}^{-1} \Psi_{n}^{*} \stackrel{a . s .}{\rightarrow} \bar{\Psi}^{*}, r_{n}^{-1} \Xi_{1 n}^{*} \rightarrow \Xi_{1}^{*}$, and $K_{n}^{-1} \Xi_{2 n}^{*} \rightarrow \Xi_{2}^{*}$ for positive definite matrices $\bar{\Psi}^{*}, \bar{\Xi}_{1}^{*}$, and $\bar{\Xi}_{2}^{*}$.

Under these assumptions, the following corollaries to Theorems 3.1 and 3.2 follow immediately, and are useful because they explicitly state the rates of convergence of the estimators under our various assumptions concerning instrument weakness.

Corollary 3.3 (JIVE1): Under Assumptions 1-7, let $\widehat{\beta}_{J I V E 1, n}$ be as defined in equation (3) above.

Then: (a) suppose that $\frac{K_{n}}{r_{n}} \rightarrow 0$ as $n \rightarrow \infty$; then,

$$
\sqrt{r_{n}}\left(\widehat{\beta}_{J I V E 1, n}-\beta_{0}\right) \stackrel{d}{\rightarrow} N\left(0, \bar{\Psi}^{-1} \bar{\Xi}_{1} \bar{\Psi}^{-1}\right), \quad \text { as } n \rightarrow \infty
$$

(b) suppose that $\frac{K_{n}}{r_{n}} \rightarrow \delta$, for $0<\delta<\infty$, as $n \rightarrow \infty$; then,

$$
\sqrt{K_{n}}\left(\widehat{\beta}_{J I V E 1, n}-\beta_{0}\right) \stackrel{d}{\rightarrow} N\left(0, \delta^{2} \bar{\Psi}^{-1}\left[\bar{\Xi}_{1}+\bar{\Xi}_{2}\right] \bar{\Psi}^{-1}\right), \quad \text { as } n \rightarrow \infty
$$

and (c) suppose that $\frac{r_{n}}{K_{n}} \rightarrow 0$ but $\frac{\sqrt{K_{n}}}{r_{n}} \rightarrow 0$ as $n \rightarrow \infty$; then,

$$
\frac{r_{n}}{\sqrt{K_{n}}}\left(\widehat{\beta}_{J I V E 1, n}-\beta_{0}\right) \stackrel{d}{\rightarrow} N\left(0, \bar{\Psi}^{-1} \bar{\Xi}_{2} \bar{\Psi}^{-1}\right), \quad \text { as } n \rightarrow \infty .
$$

Corollary 3.4 (JIVE2): Under Assumptions 1-6 and 7', let $\widehat{\beta}_{J I V E 2, n}$ be as defined in equation (4) above. Then: (a) suppose that $\frac{K_{n}}{r_{n}} \rightarrow 0$ as $n \rightarrow \infty$; then,

$$
\sqrt{r_{n}}\left(\widehat{\beta}_{J I V E 2, n}-\beta_{0}\right) \stackrel{d}{\rightarrow} N\left(0, \bar{\Psi}^{*-1} \bar{\Xi}_{2}^{*} \bar{\Psi}^{*-1}\right), \quad \text { as } n \rightarrow \infty ;
$$


(b) suppose that $\frac{K_{n}}{r_{n}} \rightarrow \delta$, for $0<\delta<\infty$, as $n \rightarrow \infty$; then,

$$
\sqrt{K_{n}}\left(\widehat{\beta}_{J I V E 2, n}-\beta_{0}\right) \stackrel{d}{\rightarrow} N\left(0, \delta^{2} \bar{\Psi}^{*-1}\left[\bar{\Xi}_{1}^{*}+\bar{\Xi}_{2}^{*}\right] \bar{\Psi}^{*-1}\right) \quad \text { as } n \rightarrow \infty
$$

and (c) suppose that $\frac{r_{n}}{K_{n}} \rightarrow 0$ but $\frac{\sqrt{K_{n}}}{r_{n}} \rightarrow 0$ as $n \rightarrow \infty$; then,

$$
\frac{r_{n}}{\sqrt{K_{n}}}\left(\widehat{\beta}_{J I V E 2, n}-\beta_{0}\right) \stackrel{d}{\rightarrow} N\left(0, \bar{\Psi}^{*-1} \bar{\Xi}_{2}^{*} \bar{\Psi}^{*-1}\right) \text { as } n \rightarrow \infty .
$$

From Corollaries 3.3 and 3.4 it is clear that the rates of convergence of both JIVE estimators depend in general on the strength of the available instruments as reflected in the relative orders of magnitude of $r_{n}$ vis-à-vis $K_{n}$. Note also that, whenever $r_{n}$ grows at a slower rate than $n$, the rate of convergence is slower than the conventional $\sqrt{n}$ rate of convergence, since in this case the available instruments are weaker than that assumed in the conventional strongly identified case, where the concentration parameter is taken to grow at the rate $n$.

Additionally, it is worth stressing that the rate of convergence in part (b) of the corrolaries corresponds to the rate found by Stock and Yogo (2003) under homoskedasticity, assuming that $r_{n}$ grows at the same rate as $K_{n}$, and for LIML, Fuller's modified LIML, and B2SLS.

Note further that in the case where $r_{n}$ grows at the same rate as $K_{n}$, the asymptotic covariance matrices of the $J I V E$ estimators involve two terms (i.e., $\bar{\Psi}^{-1} \bar{\Xi}_{1} \bar{\Psi}^{-1}$ and $\bar{\Psi}^{-1} \bar{\Xi}_{2} \bar{\Psi}^{-1}$ for $J I V E 1$ and $\bar{\Psi}^{*-1} \bar{\Xi}_{1}^{*} \bar{\Psi}^{*-1}$ and $\bar{\Psi}^{*-1} \bar{\Xi}_{2}^{*} \bar{\Psi}^{*-1}$ for $\left.J I V E 2\right)$, whereas for the other two cases the asymptotic covariance matrix only involves one or the other of these terms. This is because, as shown in the appendix, the JIVE estimators, when appropriately standardized, can be represented in terms of a linear plus a bilinear form. $\bar{\Psi}^{-1} \bar{\Xi}_{1} \bar{\Psi}^{-1}$ (alternatively, $\bar{\Psi}^{*-1} \bar{\Xi}_{1}^{*} \bar{\Psi}^{*-1}$ ) is then the asymptotic covariance matrix associated with the linear form of JIVE1 (alternatively, JIVE2) while $\bar{\Psi}^{-1} \bar{\Xi}_{2} \bar{\Psi}^{-1}$ (alternatively, $\bar{\Psi}^{*-1} \Xi_{2}^{*} \bar{\Psi}^{*-1}$ ) is the asymptotic covariance matrix associated with the bilinear form of $J I V E 1$ (alternatively, JIVE2). Now, in the case where $r_{n}$ grows faster $K_{n}$, the bilinear form is of a smaller order relative to the linear form (as is conventional asymptotics), so that only the first term appears in the formula for the asymptotic covariance matrix. On the other hand, when $r_{n}$ grows at the same rate as $K_{n}$, then the linear and the bilinear form are of the same order, so that both appears asymptotic distribution. Finally, when instrument weakness is such that $\frac{r_{n}}{K_{n}} \rightarrow 0$ but $\frac{\sqrt{K_{n}}}{r_{n}} \rightarrow 0$ as $n \rightarrow \infty$, the linear form becomes of smaller order relative to the bilinear form, so that only the second term appears in the asymptotic distribution.

Although the focus of this paper is JIVE estimators, it is important to note that at least 
some of the most well classical IV estimators are inconsistent under heteroskedasticity. In particular, consider the following estimators: (1) the Limited Infomation Maximum Likelihood (LIML) Estimator:

$$
\widehat{\beta}_{L I M L, n}=\left(Y_{2 n}^{\prime} Y_{2 n}-\widehat{\lambda}_{L I M L, n} Y_{2 n}^{\prime} M_{Z_{n}} Y_{2 n}\right)^{-1}\left(Y_{2 n}^{\prime} y_{1 n}-\widehat{\lambda}_{L I M L, n} Y_{2 n}^{\prime} M_{Z_{n}} y_{1 n}\right),
$$

where $\widehat{\lambda}_{L I M L, n}$ is the smallest root of the determinantal equation:

$$
\operatorname{det}\left\{\left(\begin{array}{cc}
y_{1 n}^{\prime} y_{1 n} & y_{1 n}^{\prime} Y_{2 n} \\
Y_{2 n}^{\prime} y_{1 n} & Y_{2 n}^{\prime} Y_{2 n}
\end{array}\right)-\lambda_{n}\left(\begin{array}{cc}
y_{1 n}^{\prime} M_{Z_{n}} y_{1 n} & y_{1 n}^{\prime} M_{Z_{n}} Y_{2 n} \\
Y_{2 n}^{\prime} M_{Z_{n}} y_{1 n} & Y_{2 n}^{\prime} M_{Z_{n}} Y_{2 n}
\end{array}\right)\right\}=0
$$

(2) the Bias-Corrected Two-Stage Least Squares (B2SLS) Estimator:

$$
\begin{aligned}
\widehat{\beta}_{B 2 S L S, n}= & \left(Y_{2 n}^{\prime} Y_{2 n}-\left(\frac{n}{n-K_{n}+2}\right) Y_{2 n}^{\prime} M_{Z_{n}} Y_{2 n}\right)^{-1} \\
& \times\left(Y_{2 n}^{\prime} y_{1 n}-\left(\frac{n}{n-K_{n}+2}\right) Y_{2 n}^{\prime} M_{Z_{n}} y_{1 n}\right) .
\end{aligned}
$$

and (3) the Two-Stage Least Squares (2SLS) Estimator:

$$
\widehat{\beta}_{2 S L S, n}=\left(Y_{2 n}^{\prime} P_{Z_{n}} Y_{2 n}\right)^{-1}\left(Y_{2 n}^{\prime} P_{Z_{n}} y_{1 n}\right)
$$

In particular, it is easy to see that none of these estimators are consistent in general under heteroskedasticity unless $r_{n}$ grows faster than $K_{n}$. To illustrate this, consider the special case where $r_{n}$ grows at the same rate as $K_{n}$, where there is only one endogenous regressor in the structural equation, where the instruments are assumed to be fixed or non-random, and where $\left\{\xi_{i}\right\} \equiv$ i.n.i.d $N\left(0, \Sigma_{i}\right)$, with $\xi_{i}=\left(u_{i}, v_{i}\right)^{\prime}$, and $\Sigma_{i}$ partitioned conformably as

$$
\Sigma_{i}=\left(\begin{array}{cc}
\sigma_{u u, i} & \sigma_{u v, i} \\
\sigma_{u v, i} & \sigma_{v v, i}
\end{array}\right) .
$$

Now, under this simplified scenario, it follows immediately via tedious but straightforward calculations that: ${ }^{4}$

$$
\begin{aligned}
\widehat{\beta}_{L I M L, n}-\beta_{0} & =\left(\bar{\Psi}_{n}+\bar{\sigma}_{v v, n}^{h}-\bar{\sigma}_{v v, n} \theta^{*}\right)^{-1}\left(\bar{\sigma}_{v v, n}^{h}-\bar{\sigma}_{v v, n} \theta^{*}\right)+o_{p}(1), \\
\widehat{\beta}_{B 2 S L S, n}-\beta_{0} & =\left(\bar{\Psi}_{n}+\bar{\sigma}_{v v, n}^{h}-\bar{\sigma}_{v v, n}\right)^{-1}\left(\bar{\sigma}_{u v, n}^{h}-\bar{\sigma}_{u v, n}\right)+o_{p}(1), \\
\widehat{\beta}_{2 S L S, n}-\beta_{0} & =\left(\bar{\Psi}_{n}+\bar{\sigma}_{v v, n}^{h}\right)^{-1} \bar{\sigma}_{u v, n}^{h}+o_{p}(1),
\end{aligned}
$$

\footnotetext{
${ }^{4}$ Detailed calculations are ommitted for the sake of brevity, and are available upon request from the authors.
} 
where

$$
\begin{aligned}
\theta_{n}^{*} & =\frac{\bar{\sigma}_{u u, n} \bar{\Psi}_{n}+\bar{\sigma}_{v v, n}^{h} \bar{\sigma}_{u u, n}+\bar{\sigma}_{u u, n}^{h} \bar{\sigma}_{v v, n}-2 \bar{\sigma}_{u v, n}^{h} \bar{\sigma}_{u v, n}-\sqrt{\psi_{n}}}{2\left(\bar{\sigma}_{u u, n} \bar{\sigma}_{v v, n}-\bar{\sigma}_{u v, n}^{2}\right)}, \\
\psi_{n} & =\left[\bar{\sigma}_{v v, n}^{h} \bar{\sigma}_{u u, n}-\bar{\sigma}_{u u, n}^{h} \bar{\sigma}_{v v, n}+\bar{\sigma}_{u u, n} \bar{\Psi}_{n}\right]^{2}+4\left(\bar{\sigma}_{u v, n} \bar{\Psi}_{n}+\bar{\sigma}_{v v, n}^{h} \bar{\sigma}_{u v, n}-\bar{\sigma}_{u v, n}^{h} \bar{\sigma}_{v v, n}\right)\left(\bar{\sigma}_{u u, n}^{h} \bar{\sigma}_{u v, n}-\bar{\sigma}_{u v, n}^{h} \bar{\sigma}_{u u,},\right.
\end{aligned}
$$$$
\bar{\sigma}_{u v, n}^{h}=\frac{1}{K_{n}} \sum_{i=1}^{n} p_{i i, n}^{Z} \sigma_{u v, i}, \bar{\sigma}_{u v, n}=\frac{1}{n} \sum_{i=1}^{n} \sigma_{u v, i}, \bar{\sigma}_{v v, n}^{h}=\frac{1}{K_{n}} \sum_{i=1}^{n} p_{i i, n}^{Z} \sigma_{u v, i}, \bar{\sigma}_{u v, n}=\frac{1}{n} \sum_{i=1}^{n} \sigma_{u v, i}, \text { and } \bar{\Psi}_{n}=
$$
$\frac{C_{n}^{\prime} Z_{n}^{\prime} Z_{n} C_{n}}{b_{n}^{2} r_{n}}$. Hence, $\widehat{\beta}_{L I M L, n}, \widehat{\beta}_{B 2 S L S, n}$ and $\widehat{\beta}_{2 S L S, n}$ are not consistent in this case. Note also that the inconsistency of $L I M L$ and $B 2 S L S$ in this case is in contrast to the homoskedasticity case where both of these estimators are consistent and asymptotically normal provided that $\frac{\sqrt{K_{n}}}{r_{n}} \rightarrow 0$ as $n \rightarrow$ $\infty$, as has been shown in Chao and Swanson $(2002,2003)$ and Stock and Yogo (2003). ${ }^{5}$ Moreover, closer inspection of the formulae given in expressions (??)-(11) shows that it is heteroskedasticity in the covariance of $u_{i}$ and $v_{i}$ which causes the inconsistency of $L I M L$ and B2SLS. Thus, if one only imposes heteroskedasticity on the error of the structural equation, as has been done in recent Monte Carlo studies of various IV estimators under weak identification, then $L I M L$ and $B 2 S L S$ will still be consistent, and thus the bias problems discussed above may not manifest themselves.

Finally, it should be noted that JIVE1 and JIVE2 are not likely to be the only estimators which will be consistent and asymptotically normal under heteroskedasticity in a many-weak-instrument framework. Estimators within the class of generalized empirical likelihood (GEL) estimators studied recently by Smith (1997) and Newey and Smith (2003) are also likely to be consistent and asymptotically normal and are likely to be more efficient than the JIVE estimators examined here. It is, thus, of interest to study the GEL estimators within our framework as well, which we intend to do for future research. However, we note that one advantage that the JIVE estimators have over the GEL estimators is their computational simplicity. As have been pointed out by Angrist, Imbens, and Krueger (1999), both JIVE estimators can be implemented easily using standard packages, whereas some of the empirical likelihood estimators are known to be difficult to compute even for simple models.allow for stochastic instruments which are not necessarily i.i.d.

\footnotetext{
${ }^{5}$ Note that Fuller's LIML estimator can also be shown to be inconsistent, using the same approach as above for examining the behavior of the LIML estimator.
} 


\section{Wald Tests for General Restrictions}

In this section we discuss the construction of Wald tests for general and possibly nonlinear coefficient restrictions. An important feature of our tests is that we introduce a new covariance matrix estimator for JIVE that is consistent, even when instruments are weak, provided that $\frac{\sqrt{K_{n}}}{r_{n}} \rightarrow 0$, as $n \rightarrow \infty$. Related work includes the tests proposed by Kleibergen (2002), Moriera (2003), and Guggenberger and Smith (2003). However, to the best of our knowledge, these tests only consider the case of testing simple null hypotheses of the form $H_{0}: \beta=\beta^{*}$, where $\beta^{*}$ is the value of $\beta$ under the null, while our approach allows for straightforward analysis of general coefficient restrictions. In addition, we allow for (unconditional) heteroskedasticity of unkown functional form. Guggenberger and Smith (2003) also address the issue of heteroskedasticity, but only consider conditional forms. However, their setup allows for nonlinear models, while our model is linear.

One of the main differences between the approach of the above authors and ours is that they assume that $K_{n}$ is fixed, and hence consistent estimation of structural parameters cannot be obtained. The advantage of their approach is that their tests still have $\chi^{2}$ limiting distributions, even when structural parameters are unidentified with respect to the class of null hypotheses that they consider. Our tests, on the other hand, are designed to handle general hypotheses of the form $H_{0}: h\left(\beta_{0}\right)=0$, where $h(\cdot): \Re^{d} \rightarrow \Re^{q}$, the caveat being that we require at least weak identification, and so does not work if the instrumentrs are completely irrelevant. In particular, in the Staiger and Stock (1997) local to zero framework, Wald test statistics do not have limiting $\chi^{2}$ distributions (the distributions are nonstandard and nuisance parameter dependent). By allowing the number of instruments to increase with the sample size, we are able to obtain consistent estimates of all parameters in our structural equation, hence leading to nuisance parameter free $\chi^{2}$ limiting distributions. Our results can thus be viewed as complimentary to those of Stiager and Stock (1997), in the sense that we consider the case of many weak instruments, while they consider the important case of few weak instruments. Indeed, all of above papers focus primarily on the case of few weak instruments. This is why, for example, Guggenberger, has noted that his test generalizes to testing sub-vectors of coefficients, although only in very specialized cases. (discussion to be completed ...)

In order to obtain asymptotic results for Wald tests, Assumptions 2-4 must be strengthened as follows.

Assumption $2^{*}$ : Let $\left\{Z_{n, i}: i=1, \ldots, n ; n \geq 1\right\}$ be a triangular array of $R^{K_{n}}$-valued random vari- 
ables, where $Z_{n, i}^{\prime}$ denotes the $i \underline{t h}$ row of the matrices $Z_{n}$. Also: (a) let $n \rightarrow \infty$, and suppose that there exist constants $\underline{D}_{\lambda}$ and $\bar{D}_{\lambda}$, with $0<\underline{D}_{\lambda} \leq \bar{D}_{\lambda}<\infty$, such that $\underline{D}_{\lambda} \leq \underline{\lim }_{n \rightarrow \infty} \lambda_{\min }\left(\frac{Z_{n}^{\prime} Z_{n}}{n}\right)$ a.s. and $\varlimsup_{n \rightarrow \infty} \lambda_{\max }\left(\frac{Z_{n}^{\prime} Z_{n}}{n}\right) \leq \bar{D}_{\lambda}$ a.s.; (b) assume that $K_{n} \rightarrow \infty$ as $n \rightarrow \infty$ such that $\frac{K_{n}^{11 / 4} n}{n} \rightarrow \kappa$, where $0 \leq \kappa<\infty$; and (c) assume that there exist constants $\underline{D}_{C}$ and $\bar{D}_{C}$, with $0<\underline{D}_{C} \leq \bar{D}_{C}<\infty$, such that $\underline{D}_{C} \leq \varliminf_{n \rightarrow \infty} \lambda_{\min }\left(\frac{C_{n}^{\prime} C_{n}}{K_{n}}\right)$ and $\varlimsup_{n \rightarrow \infty} \lambda_{\max }\left(\frac{C_{n}^{\prime} C_{n}}{K_{n}}\right) \leq \bar{D}_{C}$.

Assumption $3^{*}$ : (a) Define $\Sigma_{i}\left(Z_{n}\right)=E\left(\xi_{i} \xi_{i}^{\prime} \mid Z_{n}\right)$ and suppose that $\xi_{i} \mid Z_{n} \equiv$ i.n.i.d. $\left(0, \Sigma_{i}\left(Z_{n}\right)\right)$ almost surely for all $n$, where $\xi_{i} \mid Z_{n}$ denotes the conditional distribution of $\xi_{i}$ given $Z_{n}$. Further, assume that $\Sigma_{i}\left(Z_{n}\right)>0$ for all $i$ and $n$ almost surely, and partition $\Sigma_{i}\left(Z_{n}\right)$ conformably with $\xi_{i}=\left(u_{i}, v_{i}^{\prime}\right)^{\prime}$ as $\Sigma_{i}\left(Z_{n}\right)=\left(\begin{array}{cc}\sigma_{u u, i}\left(Z_{n}\right) & \sigma_{V u, i}^{\prime}\left(Z_{n}\right) \\ \sigma_{V u, i}\left(Z_{n}\right) & \Sigma_{V V, i}\left(Z_{n}\right)\end{array}\right)$; (b) assume that there exists some constant $\bar{D}_{\xi}$, with $0<\bar{D}_{\xi}<\infty$, such that $\max \left\{\sup _{i} E\left(u_{i}^{8} \mid Z_{n}\right), \sup _{i} E\left(v_{i 1}^{8} \mid Z_{n}\right), \ldots, \sup _{i} E\left(v_{i d}^{8} \mid Z_{n}\right)\right\} \leq \bar{D}_{\xi}$ almost surely; and (c) assume that there exists some constant $\underline{D}_{\xi}$, with $0<\underline{D}_{\xi}<\infty$, such that $\min \left\{\inf _{i} \sigma_{u u, i}\left(Z_{n}\right), \inf _{i} \lambda_{\min }\left(\Sigma_{V V, i}\left(Z_{n}\right)\right)\right\} \geq \underline{D}_{\xi}$ almost surely.

Assumption 4*: Assume that there exists a constant $D_{Z}$, with $0<D_{Z}<\infty$, such that $\sup _{1 \leq i \leq n, \leq k \leq K_{n}, n \geq 1} E\left(Z_{i k, n}^{16}\right) \leq D_{Z}$, where $Z_{i k, n}$ denotes the $(i, k)^{t h}$ element of $Z_{n}$.

Assumption 5*: Define the ratio $r_{n}=\frac{n K_{n}}{b_{n}^{2}}$. Suppose that, as $n \rightarrow \infty, \frac{r_{n}}{n} \rightarrow \kappa$ for some constant $\kappa$ such that $0 \leq \kappa<\infty$.

Notice that the above assumptions are more restrictive that Assumptions 2-4, except for Assumption $2^{*}(\mathrm{c})$ and Assumption $3^{*}(\mathrm{a})$,(c), which remain unchanged. In particular, our testing results require the number of instruments to grow more slowly, relative to the sample size, and require the existence of higher order moments than needed for normality.

Now, recall that $M_{Z_{n}}=I_{n}-Z_{n}\left(Z_{n}^{\prime} Z_{n}\right)^{-1} Z_{n}^{\prime}$. Consider general (possibly nonlinear) restrictions of the form $H_{0}: h\left(\beta_{0}\right)=0$, where $h(\cdot): \Re^{d} \rightarrow \Re^{q}$, and define the following Wald statistics:

$$
W_{J I V E 1, n}=h\left(\widehat{\beta}_{J I V E 1, n}\right)^{\prime}\left\{\mathcal{H}\left(\widehat{\beta}_{J I V E 1, n}\right)\left(\widehat{\Psi}_{n} \widehat{\Xi}_{J I V E 1, n}^{-1} \widehat{\Psi}_{n}\right)^{-1} \mathcal{H}\left(\widehat{\beta}_{J I V E 1, n}\right)^{\prime}\right\}^{-1} h\left(\widehat{\beta}_{J I V E 1, n}\right),
$$

where $\widehat{\Psi}_{n}=Y_{2 n}^{\prime}\left[I_{n}-M_{Z_{n}} H_{1 n}\right] Y_{2 n}$ and

$$
\begin{aligned}
\widehat{\Xi}_{J I V E 1, n}=Y_{2 n}^{\prime}\left[I_{n}-\right. & \left.M_{Z_{n}} H_{1 n}\right] \widehat{\Omega}_{1, n}\left[I_{n}-M_{Z_{n}} H_{1 n}\right] Y_{2 n} \\
& +\widehat{V}_{n}^{\prime} D_{1, n} H_{1 n}\left(P_{Z_{n}} \odot P_{Z_{n}}\right) H_{1 n} D_{1, n} \widehat{V}_{n} .
\end{aligned}
$$

Also,

$$
W_{J I V E 2, n}=h\left(\widehat{\beta}_{J I V E 2, n}\right)^{\prime}\left\{\mathcal{H}\left(\widehat{\beta}_{J I V E 2, n}\right)\left(\widehat{\Psi}_{n}^{*} \widehat{\Xi}_{J I V E 2, n}^{-1} \widehat{\Psi}_{n}^{*}\right)^{-1} \mathcal{H}\left(\widehat{\beta}_{J I V E 2, n}\right)^{\prime}\right\}^{-1} h\left(\widehat{\beta}_{J I V E 2, n}\right),
$$


where $\widehat{\Psi}_{n}^{*}=Y_{2 n}^{\prime}\left[P_{Z_{n}}-H_{3 n}\right] Y_{2 n}$ and

$$
\begin{aligned}
\widehat{\Xi}_{J I V E 2, n}=Y_{2 n}^{\prime}\left[P_{Z_{n}}-H_{3 n}\right] & \widehat{\Omega}_{2, n}\left[P_{Z_{n}}-H_{3 n}\right] Y_{2 n} \\
& +\widehat{V}_{n}^{\prime} D_{2, n}\left(P_{Z_{n}} \odot P_{Z_{n}}\right) D_{2, n} \widehat{V}_{n} .
\end{aligned}
$$

In the above statistics, $\mathcal{H}\left(\widehat{\beta}_{J I V E \tau, n}\right)=\left.\frac{\partial h(\beta)}{\partial \beta^{\prime}}\right|_{\beta=\widehat{\beta}_{J I V E 1, n}}, \tau=1,2, p_{i j, n}^{Z}$ denotes the $(i, j)^{t h}$ element of $P_{Z_{n}}, \widehat{u}_{J I V E \tau, i}$ is the $i^{t h}$ element of the residual vector $\widehat{u}_{J I V E \tau}=y_{1 n}-Y_{2 n} \widehat{\beta}_{J I V E \tau, n}, \widehat{v}_{i}^{\prime}$ is the $i^{t h}$ row of the residual matrix $\widehat{V}_{n}=M_{Z_{n}} Y_{2 n}, \tau=1,2$ and $\odot$ denotes the Hadamard product (see e.g. Magnus and Neudecker (1988)). Additionally,

$$
\widehat{\Omega}_{\tau, n}=\left(\begin{array}{cccc}
\widehat{u}_{J I V E \tau, 1}^{2} & 0 & \cdots & 0 \\
0 & \widehat{u}_{J I V E \tau, 2}^{2} & & \vdots \\
\vdots & & \ddots & 0 \\
0 & \cdots & 0 & \widehat{u}_{J I V E \tau, n}^{2}
\end{array}\right)
$$

and

$$
D_{\tau, n}=\left(\begin{array}{cccc}
\widehat{u}_{J I V E \tau, 1} & 0 & \cdots & 0 \\
0 & \widehat{u}_{J I V E \tau, 2} & & \vdots \\
\vdots & & \ddots & 0 \\
0 & \ldots & 0 & \widehat{u}_{J I V E \tau, n}
\end{array}\right), \quad \tau=1,2 .
$$

Note that the covariance matrix estimator, $\widehat{\Xi}_{J I V E 1, n}$ has two terms. In the proof to Theorem 4.1 below, we shall see that the first term yields a consistent estimator of $\Xi_{1, n}+\Xi_{21, n}$, while the second terms yields a consistent estimator of $\Xi_{22, n}$, where $\Xi_{1, n}$ is the covariance matrix associated with the linear part of the JIVE estimator, and where $\Xi_{2, n}$ is the covariance matrix associated with the bilinear part of the JIVE estimator (see Theorem 3.1), with $\Xi_{2, n}=\Xi_{21, n}+\Xi_{22, n}$, and

$$
\Xi_{21, n}=\sum_{1 \leq i<j \leq n}\left[g_{i j, n}^{2} \Sigma_{V V, i}\left(Z_{n}\right) \sigma_{u u, j}\left(Z_{n}\right)+g_{j i, n}^{2} \Sigma_{V V, j}\left(Z_{n}\right) \sigma_{u u, i}\left(Z_{n}\right)\right]
$$

and

$$
\Xi_{22, n}=\sum_{1 \leq i<j \leq n} g_{i j, n} g_{j i, n}\left[\sigma_{V u, i}\left(Z_{n}\right) \sigma_{V u, j}^{\prime}\left(Z_{n}\right)+\sigma_{V u, j}\left(Z_{n}\right) \sigma_{V u, i}^{\prime}\left(Z_{n}\right)\right] .
$$

Theorem 4.1: Under Assumptions $1,2^{*}-5^{*}, 6$, let $\widehat{\Xi}_{J I V E \tau, n}(\tau=1,2)$ be as defined above. Then: (a) suppose that $\frac{r_{n}}{K_{n}} \rightarrow \kappa$, where $0 \leq \kappa<\infty$, and $\frac{\sqrt{K_{n}}}{r_{n}} \rightarrow 0$, as $n \rightarrow \infty$; then, $\frac{1}{K_{n}} \widehat{\Xi}_{J I V E \tau, n}-\frac{1}{K_{n}} \Xi_{n} \stackrel{p}{\rightarrow}$ 0 , as $n \rightarrow \infty$; and (b) suppose that $\frac{K_{n}}{r_{n}} \rightarrow 0$, as $n \rightarrow \infty$; then, $\frac{1}{r_{n}} \widehat{\Xi}_{J I V E \tau, n}-\frac{1}{r_{n}} \Xi_{n} \stackrel{p}{\rightarrow} 0$, as $n \rightarrow \infty$.

Theorem 4.2: Under Assumptions $1,2^{*}-5^{*}, 6$, let $W_{J I V E \tau, n}(\tau=1,2)$ be as defined in equations () and () above. Suppose that $\frac{\sqrt{K_{n}}}{r_{n}} \rightarrow 0$ as $n \rightarrow \infty$. Then, under $H_{0}, W_{J I V E \tau, n} \stackrel{d}{\rightarrow} \chi_{q}^{2}$, as $n \rightarrow \infty$. 


\section{Monte Carlo Results}

to be completed

\section{Concluding Remarks}

to be completed 


\section{Appendix A - Estimation Results}

to be completed

We first collect some preliminary lemmas, which we will use to prove our main results.

Lemma A1: Let $p_{i j, n}^{Z}$ be the $(i, j)^{t h}$ element of the projection matrix $P_{Z_{n}}=Z_{n}\left(Z_{n}^{\prime} Z_{n}\right)^{-1} Z_{n}^{\prime}$. Then, under Assumption 2, the following statements are true as $n \rightarrow \infty$. (a) $\sum_{i=1}^{n} \sum_{j=1}^{n}\left(p_{i j, n}^{Z}\right)^{4}=O_{a . s .}\left(K_{n}\right)$;

(b) $\sum_{1 \leq i<j<k \leq n}\left(p_{i j, n}^{Z}\right)^{2}\left(p_{i k, n}^{Z}\right)^{2}=O_{a . s .}\left(K_{n}\right)$; (c) $\sum_{1 \leq i<j<k \leq n}\left(p_{i j, n}^{Z}\right)^{2}\left(p_{j k, n}^{Z}\right)^{2}=O_{a . s .}\left(K_{n}\right)$;

(d) $\sum_{1 \leq i<j<k \leq n}\left(p_{i k, n}^{Z}\right)^{2}\left(p_{j k, n}^{Z}\right)^{2}=O_{a . s .}\left(K_{n}\right)$; and (e) $\sum_{1 \leq i<j \leq n}\left(p_{i j, n}^{Z}\right)^{2}=O_{a . s .}\left(K_{n}\right)$.

Proof of Lemma A1: To show (a), observe first that the inverse $\left(Z_{n}^{\prime} Z_{n}\right)^{-1}$ exists with probability one for $n$ sufficiently large in light of Assumption 2(a). Hence, with probability one for $n$ sufficiently large, the projection matrix $P_{Z_{n}}$ is well defined, and we have

$$
\begin{aligned}
K_{n} & =\operatorname{Tr}\left(P_{Z_{n}}^{4}\right) \\
& =\sum_{1 \leq i \leq n}\left(\sum_{1 \leq j \leq n}\left(p_{i j, n}^{Z}\right)^{2}\right)^{2}+\sum_{1 \leq i<j \leq n}\left(\sum_{1 \leq k \leq n} p_{k i, n}^{Z} p_{k j, n}^{Z}\right)^{2}+\sum_{1 \leq j<i \leq n}\left(\sum_{1 \leq k \leq n} p_{k i, n}^{Z} p_{k j, n}^{Z}\right)^{2} \\
& \geq \sum_{1 \leq i \leq n}\left(\sum_{1 \leq j \leq n}\left(p_{i j, n}^{Z}\right)^{2}\right)^{2} \geq \sum_{i=1}^{n} \sum_{j=1}^{n}\left(p_{i j, n}^{Z}\right)^{4}
\end{aligned}
$$

so that $\sum_{i=1}^{n} \sum_{j=1}^{n}\left(p_{i j, n}^{Z}\right)^{4}=O_{a . s .}\left(K_{n}\right)$, as required. To show parts (b)-(d), note first that, for $n$ sufficiently large with probability one,

$$
\begin{aligned}
\operatorname{Tr}\left(P_{Z_{n}}^{4}\right) & \geq \sum_{1 \leq i \leq n}\left(\sum_{1 \leq j \leq n}\left(p_{i j, n}^{Z}\right)^{2}\right)^{2} \\
& \geq 2 \sum_{1 \leq i \leq n}\left[\sum_{1 \leq j<k \leq n}\left(p_{i j, n}^{Z}\right)^{2}\left(p_{i k, n}^{Z}\right)^{2}\right],
\end{aligned}
$$

It follows that 


$$
\begin{aligned}
O_{a . s .}\left(K_{n}\right)= & \sum_{1 \leq i \leq n}\left[\sum_{1 \leq j<k \leq n}\left(p_{i j, n}^{Z}\right)^{2}\left(p_{i k, n}^{Z}\right)^{2}\right] \\
= & 2\left\{\sum_{1 \leq i<j<k \leq n}\left(p_{i j, n}^{Z}\right)^{2}\left(p_{i k, n}^{Z}\right)^{2}+\sum_{1 \leq i<j \leq n}\left(p_{i i, n}^{Z}\right)^{2}\left(p_{i j, n}^{Z}\right)^{2}+\sum_{1 \leq i<j \leq n}\left(p_{j j, n}^{Z}\right)^{2}\left(p_{i j, n}^{Z}\right)^{2}\right. \\
& \left.+\sum_{1 \leq i<j<k \leq n}\left(p_{i j, n}^{Z}\right)^{2}\left(p_{j k, n}^{Z}\right)^{2}+\sum_{1 \leq i<j<k \leq n}\left(p_{i k, n}^{Z}\right)^{2}\left(p_{j k, n}^{Z}\right)^{2}\right\}
\end{aligned}
$$

The results stated in parts (b)-(d) then follow directly from the expression on the right-hand side of the last equality in (12) above since each term of the sum which comprises that expression is non-negative.

The proof of part (e) follows directly from the fact that, for $n$ sufficiently large such that $P_{Z_{n}}$ is well-defined with probability one, we have that $K_{n}=\operatorname{Tr}\left(P_{Z_{n}}^{2}\right)=\sum_{i=1}^{n} \sum_{j=1}^{n}\left(p_{i j, n}^{Z}\right)^{2} \geq$ $\sum_{1 \leq i<j \leq n}\left(p_{i j, n}^{Z}\right)^{2}$

\section{Lemma A2:}

Let

$$
G_{n}^{*}=P_{Z_{n}}-H_{3 n}
$$

where $H_{3 n}=\operatorname{diag}\left(p_{11, n}^{Z}, p_{22, n}^{Z}, \ldots \ldots ., p_{n n, n}^{Z}\right)$, and let $g_{i j, n}^{*}$ denote the $(i, j)^{\text {th }}$ off-diagonal element of the matrix $G_{n}^{*}$. Then, under Assumption 2, the following statements hold as $n \rightarrow \infty$

(a) $\operatorname{Tr}\left[\left(G_{n}^{*}\right)^{4}\right]=O_{\text {a.s. }}\left(K_{n}\right)$,

(b) $\sum_{1 \leq i<j<k<l \leq n}\left[p_{i k, n}^{Z} p_{j k, n}^{Z} p_{i l, n}^{Z} p_{j l, n}^{Z}+p_{i j, n}^{Z} p_{j k, n}^{Z} p_{i l, n}^{Z} p_{k l, n}^{Z}+p_{i j, n}^{Z} p_{i k, n}^{Z} p_{j l, n}^{Z} p_{k l, n}^{Z}\right]=O_{a . s .}\left(K_{n}\right)$,

(c) $\sum_{1 \leq i<j<k<l \leq n} p_{i k, n}^{Z} p_{j k, n}^{Z} p_{i l, n}^{Z} p_{j l, n}^{Z}=O_{a . s .}\left(K_{n}\right)$,

\section{Proof of Lemma A2:}


To show part (a), note that, by direct calculation,

$$
\begin{aligned}
\operatorname{Tr}\left[\left(G_{n}^{*}\right)^{4}\right]= & K_{n}-4 \operatorname{Tr}\left[P_{Z_{n}} H_{3 n}\right]+4 \operatorname{Tr}\left[P_{Z_{n}} H_{3 n}^{2}\right]+2 \operatorname{Tr}\left[P_{Z_{n}} H_{3 n} P_{Z_{n}} H_{3 n}\right] \\
& +4 \operatorname{Tr}\left[P_{Z_{n}} H_{3 n}^{3}\right]+\operatorname{Tr}\left[H_{2 n}^{4}\right] \\
\leq & K_{n}+4 \operatorname{Tr}\left[P_{Z_{n}} H_{3 n}\right]+4 \operatorname{Tr}\left[H_{3 n} P_{Z_{n}} H_{3 n}\right]+ \\
& +2 \operatorname{Tr}\left[P_{Z_{n}} H_{3 n} P_{Z_{n}} H_{3 n}\right]+4 \operatorname{Tr}\left[P_{Z_{n}} H_{3 n}^{3}\right]+\operatorname{Tr}\left[H_{2 n}^{4}\right]
\end{aligned}
$$

Note that the inverse $\left(Z_{n}^{\prime} Z_{n}\right)^{-1}$ exists with probability one for $n$ sufficiently large in light of Assumption 2(a) and, hence, $P_{Z_{n}}$ and $H_{3 n}$ are all well-defined with probability one for $n$ sufficiently large . Observe first that, with probability one for $n$ sufficiently large,

$$
\begin{aligned}
\operatorname{Tr}\left[P_{Z_{n}} H_{3 n} P_{Z_{n}} H_{3 n}\right] & =\operatorname{Tr}\left[P_{Z_{n}} H_{3 n} P_{Z_{n}} H_{3 n} P_{Z_{n}}\right] \\
& \leq \operatorname{Tr}\left[P_{Z_{n}} H_{3 n}^{2} P_{Z_{n}}\right] \\
& \leq \operatorname{Tr}\left[P_{Z_{n}}\right] \\
& =K_{n}
\end{aligned}
$$

where the first equality follows from the fact that $P_{Z_{n}}$ is idempotent, where the first inequality above follows from using the spectral decomposition of $P_{Z_{n}}$, noting the fact that since $P_{Z_{n}}$ is real, symmetric, and idempotent matrix, its eigenvalues are either 0 or 1 ; and where the second inequality follows from from the fact that $0 \leq p_{i i, n}^{Z} \leq 1$ by property of the projection matrix. By similar arguments, we note that, with probability one for $n$ sufficiently large,

$$
\begin{aligned}
\operatorname{Tr}\left[P_{Z_{n}} H_{3 n}\right] & =\sum_{i=1}^{n}\left(p_{i i, n}^{Z}\right)^{2} \leq \sum_{i=1}^{n} p_{i i, n}^{Z}=K_{n}, \\
\operatorname{Tr}\left[H_{3 n} P_{Z_{n}} H_{3 n}\right] & =\sum_{i=1}^{n}\left(p_{i i, n}^{Z}\right)^{3} \leq \sum_{i=1}^{n} p_{i i, n}^{Z}=K_{n}, \\
\operatorname{Tr}\left[P_{Z_{n}} H_{3 n}^{3}\right] & =\sum_{i=1}^{n}\left(p_{i i, n}^{Z}\right)^{4} \leq \sum_{i=1}^{n} p_{i i, n}^{Z}=K_{n}, \\
\operatorname{Tr}\left[H_{2 n}^{4}\right] & =\sum_{i=1}^{n}\left(p_{i i, n}^{Z}\right)^{4} \leq \sum_{i=1}^{n} p_{i i, n}^{Z}=K_{n} .
\end{aligned}
$$

In light of expression (13), the required result follows immediately from (14) and (15).

To show (b), observe first that by construction $G_{n}^{*}$ is a matrix such that $g_{i i, n}^{*}=0$ for $i=1, \ldots, n$ (i.e., all diagonal elements are zero) and $g_{i j, n}=p_{i j, n}^{Z}$ for $i \neq j$. Hence, for $n$ sufficiently large such that the inverse $\left(Z_{n} Z_{n}\right)^{-1}$ exists with probability one, we have 


$$
\begin{aligned}
\operatorname{Tr}\left[\left(G_{n}^{*}\right)^{4}\right]= & 2 \sum_{1 \leq i<j \leq n}\left(p_{i j, n}^{Z}\right)^{2}+2 \sum_{1 \leq k<l \leq n}\left(\sum_{1 \leq i \leq n} p_{i k, n}^{Z} p_{i l, n}^{Z}\right)^{2} \\
= & 2 \sum_{1 \leq i<j \leq n}\left(p_{i j, n}^{Z}\right)^{2} \\
& +2 \sum_{1 \leq i<j<k \leq n}\left[\left(p_{i j, n}^{Z}\right)^{2}\left(p_{i k, n}^{Z}\right)^{2}+\left(p_{i j, n}^{Z}\right)^{2}\left(p_{j k, n}^{Z}\right)^{2}+\left(p_{i k, n}^{Z}\right)^{2}\left(p_{j k, n}^{Z}\right)^{2}\right] \\
& +8 \sum_{1 \leq i<j<k<l \leq n}\left[p_{i k, n}^{Z} p_{j k, n}^{Z} p_{i l, n}^{Z} p_{j l, n}^{Z}+p_{i j, n}^{Z} p_{j k, n}^{Z} p_{i l, n}^{Z} p_{k l, n}^{Z}+p_{i j, n}^{Z} p_{i k, n}^{Z} p_{j l, n}^{Z} p_{k l, n}^{Z}\right],
\end{aligned}
$$

so that

$$
\begin{aligned}
& \left|\sum_{1 \leq i<j<k<l \leq n}\left[p_{i k, n}^{Z} p_{j k, n}^{Z} p_{i l, n}^{Z} p_{j l, n}^{Z}+p_{i j, n}^{Z} p_{j k, n}^{Z} p_{i l, n}^{Z} p_{k l, n}^{Z}+p_{i j, n}^{Z} p_{i k, n}^{Z} p_{j l, n}^{Z} p_{k l, n}^{Z}\right]\right| \\
\leq & \frac{1}{8} \operatorname{Tr}\left[\left(G_{n}^{*}\right)^{4}\right]+\frac{1}{4} \sum_{\substack{1 \leq i<j \leq n\\
}}\left(p_{i j, n}^{Z}\right)^{2} \\
& +\frac{1}{4} \sum_{\substack{1 \leq i<j<k \leq n \\
=}}\left[\left(p_{i j, n}^{Z}\right)^{2}\left(p_{i k, n}^{Z}\right)^{2}+\left(p_{i j, n}^{Z}\right)^{2}\left(p_{j k, n}^{Z}\right)^{2}+\left(k_{i k, n}^{Z}\right)^{2}\left(p_{j k, n}^{Z}\right)^{2}\right]
\end{aligned}
$$

where the last equality follows from parts (b)-(d) of Lemma A1 above and from (a) of this lemma.

To show part (c), first define the random quantities

$$
\begin{aligned}
\Delta_{1 n} & =\sum_{1 \leq i<j<k \leq n}\left[p_{i j, n}^{Z} p_{i k, n}^{Z} \varepsilon_{j} \varepsilon_{k}+p_{i j, n}^{Z} p_{j k, n}^{Z} \varepsilon_{i} \varepsilon_{k}+p_{i k, n}^{Z} p_{j k, n}^{Z} \varepsilon_{i} \varepsilon_{j}\right], \\
\Delta_{2 n} & =\sum_{1 \leq i<j<k \leq n}\left[p_{i j, n}^{Z} p_{i k, n}^{Z} \varepsilon_{j} \varepsilon_{k}+p_{i j, n}^{Z} p_{j k, n}^{Z} \varepsilon_{i} \varepsilon_{k}\right], \\
\Delta_{3 n} & =\sum_{1 \leq i<j<k \leq n} p_{i k, n}^{Z} p_{j k, n}^{Z} \varepsilon_{i} \varepsilon_{j}
\end{aligned}
$$

where we take $\left\{\varepsilon_{i}\right\}$ to be a sequence of $i . i . d$. random variables with mean 0 and variance 1 and where $\varepsilon_{i}$ and $Z_{n}$ are independent for all $i$ and $n$. Note that $\Delta_{3 n}=\Delta_{1 n}-\Delta_{2 n}$ and

$$
E\left(\Delta_{3 n}^{2} \mid Z_{n}\right)=\sum_{1 \leq i<j<k \leq n}\left(p_{i k, n}^{Z}\right)^{2}\left(p_{j k, n}^{Z}\right)^{2}+2 \sum_{1 \leq i<j<k<l \leq n} p_{i k, n}^{Z} p_{j k, n}^{Z} p_{i l, n}^{Z} p_{j l, n}^{Z} .
$$

Since $\sum_{1 \leq i<j<k \leq n}\left(p_{i k, n}^{Z}\right)^{2}\left(p_{j k, n}^{Z}\right)^{2}=O_{a . s .}\left(K_{n}\right)$ by (d) of Lemma A1, it follows that showing part (c) of this lemma is equivalent to showing that $E\left(\Delta_{3 n}^{2} \mid Z_{n}\right)=O_{a . s .}\left(K_{n}\right)$. To proceed, write

$$
E\left(\Delta_{3 n}^{2} \mid Z_{n}\right)=E\left(\Delta_{1 n}^{2} \mid Z_{n}\right)+E\left(\Delta_{2 n}^{2} \mid Z_{n}\right)-2 E\left(\Delta_{1 n} \Delta_{2 n} \mid Z_{n}\right)
$$


and, by direct calculation, we obtain

$$
\begin{aligned}
& E\left(\Delta_{1 n}^{2} \mid Z_{n}\right)=\sum_{1 \leq i<j<k \leq n}\left[\left(p_{i j, n}^{Z}\right)^{2}\left(p_{i k, n}^{Z}\right)^{2}+\left(p_{i j, n}^{Z}\right)^{2}\left(p_{j k, n}^{Z}\right)^{2}+\left(p_{i k, n}^{Z}\right)^{2}\left(p_{j k, n}^{Z}\right)^{2}\right] \\
& +4 \sum_{1 \leq i<j<k<l \leq n}\left[p_{i k, n}^{Z} p_{j k, n}^{Z} p_{i l, n}^{Z} p_{j l, n}^{Z}+p_{i j, n}^{Z} p_{j k, n}^{Z} p_{i l, n}^{Z} p_{k l, n}^{Z}+p_{i j, n}^{Z} p_{i k, n}^{Z} p_{j l, n}^{Z} p_{k l, n}^{Z}\right] \\
& E\left(\Delta_{2 n}^{2} \mid Z_{n}\right)=\sum_{1 \leq i<j<k \leq n}\left[\left(p_{i j, n}^{Z}\right)^{2}\left(p_{i k, n}^{Z}\right)^{2}+\left(p_{i j, n}^{Z}\right)^{2}\left(p_{j k, n}^{Z}\right)^{2}\right] \\
& +2 \sum_{1 \leq i<j<k<l \leq n}\left[p_{i k, n}^{Z} p_{j k, n}^{Z} p_{i l, n}^{Z} p_{j l, n}^{Z}+p_{i j, n}^{Z} p_{j k, n}^{Z} p_{i l, n}^{Z} p_{k l, n}^{Z}+p_{i j, n}^{Z} p_{i k, n}^{Z} p_{j l, n}^{Z} p_{k l, n}^{Z}\right]
\end{aligned}
$$

By parts (b)-(d) of Lemma A1 and part (b) of this lemma, we deduce that $E\left(\Delta_{1 n}^{2} \mid Z_{n}\right)=O_{a . s .}\left(K_{n}\right)$ and $E\left(\Delta_{2 n}^{2} \mid Z_{n}\right)==O_{a . s .}\left(K_{n}\right)$. It follows, by the inequality $\left|E\left(\Delta_{1 n} \Delta_{2 n} \mid Z_{n}\right)\right| \leq \frac{1}{2} E\left(\Delta_{1 n}^{2} \mid Z_{n}\right)+$ $\frac{1}{2} E\left(\Delta_{2 n}^{2} \mid Z_{n}\right)$, that

$$
\begin{aligned}
E\left(\Delta_{3 n}^{2} \mid Z_{n}\right) & \leq 2 E\left(\Delta_{1 n}^{2} \mid Z_{n}\right)+2 E\left(\Delta_{2 n}^{2} \mid Z_{n}\right) \\
& =O_{\text {a.s. }}\left(K_{n}\right) .
\end{aligned}
$$

Lemma A3: Let $P_{Z_{n}}^{U}$ be the upper triangular matrix defined by

$$
P_{Z_{n}}^{U}=\left(\begin{array}{ccccc}
0 & p_{12, n}^{Z} & & & p_{1 n, n}^{Z} \\
0 & 0 & & & \vdots \\
\vdots & & \ddots & & \vdots \\
& & & 0 & p_{n-1 n, n}^{Z} \\
0 & \cdots & \cdots & 0 & 0
\end{array}\right),
$$

where $p_{i j, n}^{Z}$ denotes the $(i, j)^{\text {th }}$ element of the projection matrix $P_{Z_{n}}$. Then, under Assumption 2, $\left\|\left(P_{Z_{n}}^{U}\right)^{\prime} P_{Z_{n}}^{U}\right\|_{F}=O_{\text {a.s. }}\left(\sqrt{K_{n}}\right)$ as $n \rightarrow \infty$, where $\|\cdot\|_{F}$ here denotes the Frobenius matrix norm so that $\|A\|_{F}=\left[\operatorname{Tr}\left(A^{\prime} A\right)\right]^{\frac{1}{2}}$.

\section{Proof of Lemma A3:}

By direct calculation, we obtain

$$
\begin{aligned}
\left\|\left(P_{Z_{n}}^{U}\right)^{\prime} P_{Z_{n}}^{U}\right\|_{F}^{2}= & \operatorname{Tr}\left(\left(P_{Z_{n}}^{U}\right)^{\prime} P_{Z_{n}}^{U}\left(P_{Z_{n}}^{U}\right)^{\prime} P_{Z_{n}}^{U}\right) \\
= & \sum_{1 \leq i<j \leq n}\left(p_{i j, n}^{Z}\right)^{4}+4 \sum_{1 \leq i<j<k<l \leq n} p_{i k, n}^{Z} p_{j k, n}^{Z} p_{i l, n}^{Z} p_{j l, n}^{Z} \\
& +2 \sum_{1 \leq i<j<k \leq n}\left[\left(p_{i j, n}^{Z}\right)^{2}\left(p_{i k, n}^{Z}\right)^{2}+\left(p_{i k, n}^{Z}\right)^{2}\left(p_{j k, n}^{Z}\right)^{2}\right] \\
= & O_{a . s .}\left(K_{n}\right) .
\end{aligned}
$$


where the last equality follows from parts (a), (b), and (d) of Lemma A1 and part (c) of Lemma A2. It follows immediately that $\left\|\left(P_{Z_{n}}^{U}\right)^{\prime} P_{Z_{n}}^{U}\right\|_{F}=O_{a . s .}\left(\sqrt{K_{n}}\right)$.

Lemma A4: Let

$$
\begin{aligned}
\Xi_{n}= & \sum_{j=1}^{n} b_{n}^{-2} C_{n}^{\prime} Z_{n, j} Z_{n, i}^{\prime} C_{n} \sigma_{u u, j}\left(Z_{n}\right) \\
& +\sum_{\substack{1 \leq i<j \leq n\\
}}\left[g_{i j, n}^{2} \Sigma_{V V, i}\left(Z_{n}\right) \sigma_{u u, j}\left(Z_{n}\right)+g_{i j, n} g_{j i, n} \sigma_{V u, i}\left(Z_{n}\right) \sigma_{V u, j}^{\prime}\left(Z_{n}\right)\right. \\
& \left.+g_{i j, n} g_{j i, n} \sigma_{V u, j}\left(Z_{n}\right) \sigma_{V u, i}^{\prime}\left(Z_{n}\right)+g_{j i, n}^{2} \Sigma_{V V, j}\left(Z_{n}\right) \sigma_{u u, i}\left(Z_{n}\right)\right],
\end{aligned}
$$

where $g_{i j, n}$ denote the $(i, j)^{\text {th }}$ off-diagonal element of the matrix

$$
G_{n}=P_{Z_{n}} H_{1 n}-H_{2 n}
$$

where $H_{1 n}=\operatorname{diag}\left(\frac{1}{1-p_{11, n}^{Z}}, \frac{1}{1-p_{22, n}^{Z}}, \ldots \ldots ., \frac{1}{1-p_{n n, n}^{Z}}\right)$ and $H_{2 n}=\operatorname{diag}\left(\frac{p_{11, n}^{Z}}{1-p_{11, n}^{Z}}, \frac{p_{22, n}^{Z}}{1-p_{22, n}^{Z}}, \ldots \ldots ., \frac{p_{n n, n}^{Z}}{1-p_{n n, n}^{Z}}\right)$. Suppose that Assumptions 1-6 hold and suppose that $\frac{r_{n}}{K_{n}} \rightarrow \kappa$ for $0 \leq \kappa<\infty$ but $\frac{\sqrt{K_{n}}}{r_{n}} \rightarrow 0$ as $n \rightarrow \infty$. Then,

(a) $\Xi_{n} \asymp K_{n}$, i.e., $\Xi_{n}=O_{a . s .}\left(K_{n}\right)$ and $\Xi_{n}$ is not of an order lower than $K_{n}$.

(b) $\Xi_{n}$ is positive definite with probability one for $n$ sufficiently large.

\section{Proof of Lemma A4:}

Note first that it suffices that we show $\gamma^{\prime} \Xi_{n} \gamma \asymp K_{n}$ for all vectors $\gamma \in R^{d}$ such that $\|\gamma\|=1$. To proceed, let $\tau_{n}=\gamma^{\prime} \Xi_{n} \gamma$ and note that, with probability one, 


$$
\begin{aligned}
\tau_{n}= & \sum_{j=1}^{n} b_{n}^{-2} \gamma^{\prime} C_{n}^{\prime} Z_{n, j} Z_{n, i}^{\prime} C_{n} \gamma \sigma_{u u, j}\left(Z_{n}\right) \\
& +\sum_{1 \leq i<j \leq n}\left[g_{i j, n}^{2} \gamma^{\prime} \Sigma_{V V, i}\left(Z_{n}\right) \gamma \sigma_{u u, j}\left(Z_{n}\right)+g_{i j, n} g_{j i, n} \gamma^{\prime} \sigma_{V u, i}\left(Z_{n}\right) \sigma_{V u, j}^{\prime}\left(Z_{n}\right) \gamma\right. \\
& \left.+g_{i j, n} g_{j i, n} \gamma^{\prime} \sigma_{V u, j}\left(Z_{n}\right) \sigma_{V u, i}^{\prime}\left(Z_{n}\right) \gamma+g_{j i, n}^{2} \gamma^{\prime} \Sigma_{V V, j}\left(Z_{n}\right) \gamma \sigma_{u u, i}\left(Z_{n}\right)\right] \\
\leq & \bar{D}_{\xi}^{\frac{1}{2}} \sum_{j=1}^{n} b_{n}^{-2} \gamma^{\prime} C_{n}^{\prime} Z_{n, j} Z_{n, i}^{\prime} C_{n} \gamma \\
& +d \bar{D}_{\xi} \sum_{1 \leq i<j \leq n}\left[g_{i j, n}^{2}+2 g_{i j, n} g_{j i, n}+g_{j i, n}^{2}\right] \\
\leq & \bar{D}_{\xi}^{\frac{1}{2}} \frac{\gamma^{\prime} C_{n}^{\prime} Z_{n}^{\prime} Z_{n} C_{n} \gamma}{b_{n}^{2}}+2 d \bar{D}_{\xi} \sum_{1 \leq i<j \leq n}\left[g_{i j, n}^{2}+g_{j i, n}^{2}\right] \\
\leq & \bar{D}_{\xi}^{\frac{1}{2}} \frac{\gamma^{\prime} C_{n}^{\prime} Z_{n}^{\prime} Z_{n} C_{n} \gamma}{b_{n}^{2}}+4 d \bar{D}_{\xi}\left(\frac{1}{1-\bar{h}}\right)_{1 \leq i<j \leq n}\left(p_{i j, n}^{Z}\right)^{2}
\end{aligned}
$$

where the last inequality follows from Assumption 6. It follows immediately from the assumption that $\frac{r_{n}}{K_{n}} \rightarrow \kappa$ for $0 \leq \kappa<\infty$ and from part (e) of Lemma A1 that $\tau_{n}=O_{a . s .}\left(K_{n}\right)$.

Next, we show that $\tau_{n}$ is not of an order lower than $K_{n}$. To proceed, note that

$$
\begin{aligned}
\tau_{n}= & \sum_{j=1}^{n} b_{n}^{-2} \gamma^{\prime} C_{n}^{\prime} Z_{n, j} Z_{n, i}^{\prime} C_{n} \gamma \sigma_{u u, j}\left(Z_{n}\right) \\
& +\sum_{1 \leq i<j \leq n}\left[g_{i j, n}^{2} \gamma^{\prime} \Sigma_{V V, i}\left(Z_{n}\right) \gamma \sigma_{u u, j}\left(Z_{n}\right)+g_{i j, n} g_{j i, n} \gamma^{\prime} \sigma_{V u, i}\left(Z_{n}\right) \sigma_{V u, j}^{\prime}\left(Z_{n}\right) \gamma\right. \\
& \left.+g_{i j, n} g_{j i, n} \gamma^{\prime} \sigma_{V u, j}\left(Z_{n}\right) \sigma_{V u, i}^{\prime}\left(Z_{n}\right) \gamma+g_{j i, n}^{2} \gamma^{\prime} \Sigma_{V V, j}\left(Z_{n}\right) \gamma \sigma_{u u, i}\left(Z_{n}\right)\right] \\
= & \sum_{j=1}^{n} b_{n}^{-2} \gamma^{\prime} C_{n}^{\prime} Z_{n, j} Z_{n, i}^{\prime} C_{n} \gamma \sigma_{u u, j}\left(Z_{n}\right) \\
& +\sum_{1 \leq i<j \leq n} E_{Z}\left[( p _ { i j , n } ^ { Z } ) ^ { 2 } \left\{\left(1-p_{j j, n}^{Z}\right)^{-2} \gamma^{\prime} \Sigma_{V V, i}\left(Z_{n}\right) \gamma \sigma_{u u, j}\left(Z_{n}\right)\right.\right. \\
& +\left(1-p_{i i, n}^{Z}\right)^{-2} \gamma^{\prime} \Sigma_{V V, j}\left(Z_{n}\right) \gamma \sigma_{u u, i}\left(Z_{n}\right) \\
& \left.\left.+2\left(\left(1-p_{i i, n}^{Z}\right)\left(1-p_{j j, n}^{Z}\right)\right)^{-1} \gamma^{\prime} \sigma_{V u, j}\left(Z_{n}\right) \sigma_{V u, i}^{\prime}\left(Z_{n}\right) \gamma\right\}\right]
\end{aligned}
$$


Now, note that with probability one,

$$
\begin{aligned}
& \left(1-p_{j j, n}^{Z}\right)^{-2} \gamma^{\prime} \Sigma_{V V, i}\left(Z_{n}\right) \gamma \sigma_{u u, j}\left(Z_{n}\right)+\left(1-p_{i i, n}^{Z}\right)^{-2} \gamma^{\prime} \Sigma_{V V, j}\left(Z_{n}\right) \gamma \sigma_{u u, i}\left(Z_{n}\right) \\
& +2\left(\left(1-p_{i i, n}^{Z}\right)\left(1-p_{j j, n}^{Z}\right)\right)^{-1} \gamma^{\prime} \sigma_{V u, j}\left(Z_{n}\right) \sigma_{V u, i}^{\prime}\left(Z_{n}\right) \gamma \\
> & \left(1-p_{j j, n}^{Z}\right)^{-2} \gamma^{\prime} \Sigma_{V V, i}\left(Z_{n}\right) \gamma \sigma_{u u, j}\left(Z_{n}\right)+\left(1-p_{i i, n}^{Z}\right)^{-2} \gamma^{\prime} \Sigma_{V V, j}\left(Z_{n}\right) \gamma \sigma_{u u, i}\left(Z_{n}\right) \\
& -2\left(\left(1-p_{i i, n}^{Z}\right)\left(1-p_{j j, n}^{Z}\right)\right)^{-1} \sqrt{\sigma_{u u, i}\left(Z_{n}\right)} \sqrt{\gamma^{\prime} \Sigma_{V V, i}\left(Z_{n}\right) \gamma} \sqrt{\sigma_{u u, j}\left(Z_{n}\right)} \sqrt{\gamma^{\prime} \Sigma_{V V, i}\left(Z_{n}\right) \gamma} \\
= & {\left[\left(1-p_{j j, n}^{Z}\right)^{-1} \sqrt{\gamma^{\prime} \Sigma_{V V, i}\left(Z_{n}\right) \gamma} \sqrt{\sigma_{u u, j}\left(Z_{n}\right)}-\left(1-p_{i i, n}^{Z}\right)^{-1} \sqrt{\sigma_{u u, i}\left(Z_{n}\right)} \sqrt{\gamma^{\prime} \Sigma_{V V, j}\left(Z_{n}\right) \gamma}\right]^{2} } \\
\geq & 0,
\end{aligned}
$$

where the first inequality follows from the Cauchy-Schwarz inequality and where the inequality is strict because under Assumption, $\gamma^{\prime} V_{i, n}$ is not a linear function of $u_{i}$ almost surely. Thus, there exists a constant $\varpi>0$ such that, with probability one,

$$
\begin{aligned}
& \left(1-p_{j j, n}^{Z}\right)^{-2} \gamma^{\prime} \Sigma_{V V, i}\left(Z_{n}\right) \gamma \sigma_{u u, j}\left(Z_{n}\right)+\left(1-p_{i i, n}^{Z}\right)^{-2} \gamma^{\prime} \Sigma_{V V, j}\left(Z_{n}\right) \gamma \sigma_{u u, i}\left(Z_{n}\right) \\
& +2\left(\left(1-p_{i i, n}^{Z}\right)\left(1-p_{j j, n}^{Z}\right)\right)^{-1} \gamma^{\prime} \sigma_{V u, j}\left(Z_{n}\right) \sigma_{V u, i}^{\prime}\left(Z_{n}\right) \gamma \\
\geq & \varpi \\
> & 0 .
\end{aligned}
$$

It follows that

$$
\begin{aligned}
\tau_{n} & \geq \sum_{j=1}^{n} b_{n}^{-2} \gamma^{\prime} C_{n}^{\prime} Z_{n, j} Z_{n, i}^{\prime} C_{n} \gamma \sigma_{u u, j}\left(Z_{n}\right)+\varpi \sum_{1 \leq i<j \leq n}\left(p_{i j, n}^{Z}\right)^{2} \\
& \geq \underline{D}_{\xi} \sum_{j=1}^{n} b_{n}^{-2} \gamma^{\prime} C_{n}^{\prime} Z_{n, j} Z_{n, i}^{\prime} C_{n} \gamma+\frac{\varpi}{2} \sum_{i=1} p_{i i, n}^{Z}\left(1-p_{i i, n}^{Z}\right) \\
& \geq \underline{D}_{\xi} \frac{\gamma^{\prime} C_{n}^{\prime} Z_{n}^{\prime} Z_{n} C_{n} \gamma}{b_{n}^{2}}+\frac{\varpi}{2}(1-\bar{h}) \sum_{i=1} p_{i i, n}^{Z} \\
& =\underline{D}_{\xi} \frac{\gamma^{\prime} C_{n}^{\prime} Z_{n}^{\prime} Z_{n} C_{n} \gamma}{b_{n}^{2}}+\frac{\varpi}{2}(1-\bar{h}) K_{n} .
\end{aligned}
$$

The desired result follows immediately.

The proof of part (b) follows immediately in light of (18) and (19) and Assumptions 2(a), 2(c), $3(\mathrm{c})$, and 6 .

Lemma A5: Let $G_{n}$ and $g_{i j, n}$ be as defined in Lemma A4. Then, under Assumptions 2 and 6, the following statements are true 

(a) $\frac{1}{K_{n}^{2}} \sum_{1 \leq i<j \leq n} g_{i j, n}^{4}=o_{a . s .}(1)$
(b) $\frac{1}{K_{n}^{2}} \sum_{1 \leq i<j \leq n} g_{j i, n}^{4}=o_{a . s .}(1)$
(c) $\frac{1}{K_{n}^{2}} \sum_{1 \leq i<j \leq n} g_{i j, n}^{2} g_{j i, n}^{2}=o_{a . s .}(1)$
(d) $\frac{1}{K_{n}^{2}} \sum_{1 \leq i<j<k \leq n} g_{i k, n}^{2} g_{j k, n}^{2}=o_{a . s .}(1)$
(e) $\frac{1}{K_{n}^{2}} \sum_{1 \leq i<j<k \leq n} g_{k i, n}^{2} g_{j k, n}^{2}=o_{a . s .}(1)$
(f) $\frac{1}{K_{n}^{2}} \sum_{1 \leq i<j<k \leq n} g_{k i, n}^{2} g_{k j, n}^{2}=o_{a . s .}(1)$
(g) $\frac{1}{K_{n}^{2}} \sum_{1 \leq i<j<k \leq n} g_{k i, n}^{2} g_{j k, n}^{2}=o_{a . s .}(1)$

\section{Proof of Lemma A5:}

To show part (a), observe first that the inverse $\left(Z_{n}^{\prime} Z_{n}\right)^{-1}$ exists with probability one for $n$ sufficiently large in light of Assumption 2(a). Hence, with probability one for $n$ sufficiently large, the projection matrix $P_{Z_{n}}$ is well defined, and we have

$$
\begin{aligned}
\frac{1}{K_{n}^{2}} \sum_{1 \leq i<j \leq n} g_{i j, n}^{4} & =\frac{1}{K_{n}^{2}} \sum_{1 \leq i<j \leq n} \frac{\left(p_{i j, n}^{Z}\right)^{4}}{\left(1-p_{j j, n}^{Z}\right)^{4}} \\
& \leq\left(\frac{1}{1-\bar{h}}\right)^{4} \frac{1}{K_{n}^{2}} \sum_{1 \leq i<j \leq n}\left(p_{i j, n}^{Z}\right)^{4} \\
& \leq\left(\frac{1}{1-\bar{h}}\right)^{4} \frac{1}{K_{n}^{2}} \sum_{i=1}^{n} \sum_{j=1}^{n}\left(p_{i j, n}^{Z}\right)^{4} \\
& =o_{a . s .}(1),
\end{aligned}
$$

where the first inequality follows from Assumption 6 and the last equality follows from applying part (a) of Lemma A1. All other parts of this lemma can be proved in a similar way using Lemma A1. For the sake of brevity, we omit these proofs.

Lemma A6: Let $G_{n}$ be defined as in (17) Also, let

$$
\begin{aligned}
f_{n} & =b_{n}^{-1} Z_{n} C_{n} \bar{\Psi}_{n}^{-1}\left(\bar{\Psi}_{n}^{-1} \bar{\Xi}_{n} \bar{\Psi}_{n}^{-1}\right)^{-\frac{1}{2}} \gamma \\
\eta_{n} & =V_{n} \bar{\Psi}_{n}^{-1}\left(\bar{\Psi}_{n}^{-1} \bar{\Xi}_{n} \bar{\Psi}_{n}^{-1}\right)^{-\frac{1}{2}} \gamma,
\end{aligned}
$$


and let $f_{j, n}$ and $\eta_{j, n}$ be the $j^{\text {th }}$ element of $f_{n}$ and $\eta_{n}$, respectively, where $\bar{\Psi}_{n}=\frac{C_{n}^{\prime} Z_{n}^{\prime} Z_{n} C_{n}}{b_{n}^{2} r_{n}}$, where $\Xi_{n}=K_{n}^{-1} \Xi_{n}$, with $\Xi_{n}$ as defined in (16), and $\gamma \in R^{d}$ such that $\|\gamma\|=1$. Futhermore, let

$$
\begin{aligned}
\sigma_{u u, j}\left(Z_{n}\right) & =E\left(u_{i}^{2} \mid Z_{n}\right), \\
\sigma_{\eta u, j}\left(Z_{n}\right) & =E\left(\eta_{i, n} u_{i}^{2} \mid Z_{n}\right), \\
\sigma_{\eta \eta, j}\left(Z_{n}\right) & =E\left(\eta_{i, n}^{2} \mid Z_{n}\right) .
\end{aligned}
$$

Under Assumptions 1-6, suppose that $r_{n} \rightarrow \infty$ as $n \rightarrow \infty$ such that $\frac{r_{n}}{K_{n}} \rightarrow \kappa$, where $\kappa$ is a constant such that $0<\kappa<\infty$. Then, the following statements are true as $n \rightarrow \infty$ :
(a) $\frac{1}{K_{n}^{2}} \sum_{j=1}^{n} E_{Z_{n}}\left(f_{j, n}^{4}\right)=o(1)$
(b) $\frac{1}{K_{n}^{2}} \sum_{1 \leq i<j \leq n} f_{j, n}^{2} g_{i j, n}^{2}=o_{a . s .}(1)$,
(c) $\frac{1}{K_{n}^{2}} \sum_{1 \leq i<j \leq n} f_{j, n}^{2} g_{j i, n}^{2}=o_{a . s .}(1)$,
(d) $\frac{1}{K_{n}^{2}} \sum_{1 \leq i<j \leq n} f_{j, n} g_{i j, n}^{3}=o_{a . s .}(1)$,
(e) $\frac{1}{K_{n}^{2}} \sum_{1 \leq i<j<k \leq n} f_{j, n} f_{k, n} g_{i j, n} g_{i k, n} \sigma_{\eta \eta, i}\left(Z_{n}\right) \sigma_{u u, j}\left(Z_{n}\right) \sigma_{u u, k}\left(Z_{n}\right)=o_{a . s .}(1)$,
(f) $\frac{1}{K_{n}^{2}} \sum_{1 \leq i<j<k \leq n} f_{j, n} f_{k, n} g_{j i, n} g_{k i, n} \sigma_{u u, i}\left(Z_{n}\right) \sigma_{\eta u, j}\left(Z_{n}\right) \sigma_{\eta u, k}\left(Z_{n}\right)=o_{a . s .}(1)$,
(g) $\frac{1}{K_{n}^{2}} \sum_{1 \leq i<j<k \leq n} f_{j, n} f_{k, n} g_{i j, n} g_{k i, n} \sigma_{\eta u, i}\left(Z_{n}\right) \sigma_{u u, j}\left(Z_{n}\right) \sigma_{\eta u, k}\left(Z_{n}\right)=o_{a . s .}(1)$,
(h) $\frac{1}{K_{n}^{2}} \sum_{1 \leq i<j<k \leq n} f_{j, n} f_{k, n} g_{j i, n} g_{i k, n} \sigma_{\eta u, i}\left(Z_{n}\right) \sigma_{\eta u, j}\left(Z_{n}\right) \sigma_{u u, k}\left(Z_{n}\right)=o_{a . s .}(1)$.

\section{Proof of Lemma A6:}

To show part (a), note first that, by the Cauchy-Schwarz inequality, we have that with probability one

$$
\begin{aligned}
\left|f_{j, n}\right|= & \left|b_{n}^{-1}\left(1-p_{j j, n}^{Z}\right) \gamma^{\prime}\left(\bar{\Psi}_{n}^{-1} \bar{\Xi}_{n} \bar{\Psi}_{n}^{-1}\right)^{-\frac{1}{2}} \bar{\Psi}_{n}^{-1} C_{n}^{\prime} Z_{j, n}\right| \\
\leq & {\left[b_{n}^{-2}\left(1-p_{j j, n}^{Z}\right)^{2} Z_{j, n}^{\prime} C_{n} \bar{\Psi}_{n}^{-1} C_{n}^{\prime} Z_{j, n}\right]^{\frac{1}{2}} } \\
& \times\left[\gamma^{\prime}\left(\bar{\Psi}_{n}^{-1} \bar{\Xi}_{n} \bar{\Psi}_{n}^{-1}\right)^{-\frac{1}{2}} \bar{\Psi}_{n}^{-1}\left(\bar{\Psi}_{n}^{-1} \bar{\Xi}_{n} \bar{\Psi}_{n}^{-1}\right)^{-\frac{1}{2}} \gamma\right]^{\frac{1}{2}},
\end{aligned}
$$


Note that Lemma A4 and Assumption 2 imply that, for $n$ sufficiently large, there exists a constant $D_{7}$ such that, with probability one

$$
\gamma^{\prime}\left(\bar{\Psi}_{n}^{-1} \bar{\Xi}_{n} \bar{\Psi}_{n}^{-1}\right)^{-\frac{1}{2}} \bar{\Psi}_{n}^{-1}\left(\bar{\Psi}_{n}^{-1} \bar{\Xi}_{n} \bar{\Psi}_{n}^{-1}\right)^{-\frac{1}{2}} \gamma \leq D_{7} .
$$

Moreover, with probability one,

$$
b_{n}^{-2}\left(1-p_{j j, n}^{Z}\right)^{2} Z_{j, n}^{\prime} C_{n} \bar{\Psi}_{n}^{-1} C_{n}^{\prime} Z_{j, n} \leq\left(\frac{K_{n}}{b_{n}^{2}}\right)\left(\frac{1}{\lambda_{\min }\left(\bar{\Psi}_{n}\right)}\right) \lambda_{\max }\left(\frac{C_{n}^{\prime} C_{n}}{K_{n}}\right) Z_{j, n}^{\prime} Z_{j, n} .
$$

It follows from (25) and (26) and Assumption 4 that

$$
\begin{aligned}
\frac{1}{K_{n}^{2}} \sum_{j=1}^{n} E_{Z_{n}}\left(f_{j, n}^{4}\right) \leq & \left(\frac{1}{b_{n}^{2}}\right)^{2}\left(\frac{1}{\lambda_{\min }\left(\bar{\Psi}_{n}\right)}\right)^{2}\left[\lambda_{\max }\left(\frac{C_{n}^{\prime} C_{n}}{K_{n}}\right)\right]^{2} \\
& \times D_{7}^{2} \sum_{j=1}^{n} E_{Z_{n}}\left(Z_{j, n}^{\prime} Z_{j, n}\right)^{2} \\
= & \left(\frac{1}{m_{n}}\right)^{2}\left(\frac{1}{\lambda_{\min }\left(\bar{\Psi}_{n}\right)}\right)^{2}\left[\lambda_{\max }\left(\frac{C_{n}^{\prime} C_{n}}{K_{n}}\right)\right]^{2} D_{7}^{2} \\
& \times \sum_{j=1}^{n} \sum_{k=1}^{K_{n}} \sum_{l=1}^{K_{n}} E_{Z_{n}}\left(Z_{j k, n}^{2} Z_{j l, n}^{2}\right) \\
\leq & \frac{K_{n}^{2} n}{b_{n}^{4}}\left(\frac{1}{\lambda_{\min }\left(\bar{\Psi}_{n}\right)}\right)^{2}\left[\lambda_{\max }\left(\frac{C_{n}^{\prime} C_{n}}{K_{n}}\right)\right]^{2} D_{7}^{2} D_{Z} \\
= & O\left(\frac{K_{n}^{2} n}{m_{n}^{2}}\right),
\end{aligned}
$$

where the order of magnitude follows from the fact that $\frac{m_{n}}{b_{n}^{2}}=O(1)$ in this case.

To show part (b), note that 


$$
\begin{aligned}
\frac{1}{K_{n}^{2}} \sum_{1 \leq i<j \leq n} f_{j, n}^{2} g_{i j, n}^{2} \leq & \frac{1}{K_{n}^{2}} \sum_{i=1}^{n} \sum_{j=1}^{n} f_{j, n}^{2} g_{i j, n}^{2} \\
= & \frac{r_{n}}{K_{n}^{2}}\left(\frac{1}{r_{n}}\right) \sum_{j=1}^{n} f_{j, n}^{2} \sum_{i=1}^{n} g_{i j, n}^{2} \\
\leq & \frac{r_{n}}{K_{n}^{2}}\left[\left(\frac{1}{r_{n}}\right) \sum_{j=1}^{n} f_{j, n}^{2} p_{j j}^{Z}\right] \\
\leq & \left(\frac{r_{n}}{K_{n}^{2}}\right)\left(1-p_{j j, n}^{Z}\right) \gamma^{\prime}\left(\bar{\Psi}_{n}^{-1} \bar{\Xi}_{n} \bar{\Psi}_{n}^{-1}\right)^{-\frac{1}{2}} \bar{\Psi}_{n}^{-1}\left(\frac{C_{n}^{\prime} Z_{n}^{\prime} Z_{n} C_{n}}{b_{n}^{2} r_{n}}\right) \\
& \times \bar{\Psi}_{n}^{-1}\left(\bar{\Psi}_{n}^{-1} \bar{\Xi}_{n} \bar{\Psi}_{n}^{-1}\right)^{-\frac{1}{2}} \gamma \\
\leq & \left(\frac{r_{n}}{K_{n}^{2}}\right) \gamma^{\prime}\left(\bar{\Psi}_{n}^{-1} \bar{\Xi}_{n} \bar{\Psi}_{n}^{-1}\right)^{-\frac{1}{2}} \bar{\Psi}_{n}^{-1}\left(\frac{C_{n}^{\prime} Z_{n}^{\prime} Z_{n} C_{n}}{b_{n}^{2} r_{n}}\right) \\
& \times \bar{\Psi}_{n}^{-1}\left(\bar{\Psi}_{n}^{-1} \bar{\Xi}_{n} \bar{\Psi}_{n}^{-1}\right)^{-\frac{1}{2}} \gamma \\
= & O_{a . s .}\left(\frac{r_{n}}{K_{n}^{2}}\right)
\end{aligned}
$$

It follows that

$$
\frac{1}{K_{n}^{2}} \sum_{1 \leq i<j \leq n} E_{Z_{n}}\left(f_{j, n}^{2} g_{i j, n}^{2}\right)=O\left(\frac{r_{n}}{K_{n}^{2}}\right)=o(1) .
$$

Part (c) can be show in a manner similar to part (b). Hence, for the sake of brevity, we omit the proof.

To show part (d), note that part (a) of Lemma A5 and part (b) of this lemma imply that

$$
\begin{aligned}
\frac{1}{K_{n}^{2}}\left|\sum_{1 \leq i<j \leq n} f_{j, n} g_{i j, n}^{3}\right| & \leq \frac{1}{K_{n}^{2}} \sum_{1 \leq i<j \leq n}\left|f_{j, n} g_{i j, n}^{3}\right| \\
& \leq \frac{1}{K_{n}^{2}} \sum_{1 \leq i<j \leq n} \frac{1}{2}\left(f_{j, n}^{2}+g_{i j, n}^{2}\right) g_{i j, n}^{2} \\
& =\left(\frac{1}{2}\right) \frac{1}{K_{n}^{2}} \sum_{1 \leq i<j \leq n} f_{j, n}^{2} g_{i j, n}^{2}+\left(\frac{1}{2}\right) \frac{1}{K_{n}^{2}} \sum_{1 \leq i<j \leq n} g_{i j, n}^{4} \\
& =o_{a . s .}(1) .
\end{aligned}
$$


To show (e), note first that

$$
\begin{aligned}
& \sum_{1 \leq i<j<k \leq n} f_{j, n} f_{k, n} g_{i j, n} g_{i k, n} \sigma_{\eta \eta, i}\left(Z_{n}\right) \sigma_{u u, j}\left(Z_{n}\right) \sigma_{u u, k}\left(Z_{n}\right) \\
= & \frac{1}{2} f_{n}^{\prime} \Omega_{u u, n}\left(Z_{n}\right) H_{1 n}\left(P_{Z_{n}}^{U}\right)^{\prime} \Omega_{\eta \eta, n}\left(Z_{n}\right) P_{Z_{n}}^{U} H_{1 n} \Omega_{u u, n}\left(Z_{n}\right) f_{n}- \\
& \frac{1}{2} \sum_{1 \leq i<j \leq n} f_{j, n}^{2} g_{i j, n}^{2} \sigma_{\eta \eta, i}\left(Z_{n}\right) \sigma_{u u, j}^{2}\left(Z_{n}\right),
\end{aligned}
$$

where

$$
\begin{aligned}
\Omega_{u u, n}\left(Z_{n}\right)= & \left(\begin{array}{ccccc}
\sigma_{u u, 1}\left(Z_{n}\right) & 0 & \cdots & \cdots & 0 \\
0 & \sigma_{u u, 2}\left(Z_{n}\right) & 0 & & \vdots \\
0 & 0 & \ddots & \ddots & \vdots \\
\vdots & & \ddots & \sigma_{u u, n-1}\left(Z_{n}\right) & 0 \\
0 & 0 & \cdots & 0 & \sigma_{u u, n}\left(Z_{n}\right)
\end{array}\right), \\
\Omega_{\eta \eta, n}\left(Z_{n}\right)= & \left(\begin{array}{ccccc}
\sigma_{\eta \eta, 1}\left(Z_{n}\right) & 0 & \cdots & \cdots & 0 \\
0 & \sigma_{\eta \eta, 2}\left(Z_{n}\right) & 0 & & \vdots \\
0 & 0 & \ddots & \ddots & \vdots \\
\vdots & & \ddots & \sigma_{\eta \eta, n-1}\left(Z_{n}\right) & 0 \\
0 & 0 & \cdots & 0 & \sigma_{\eta \eta, n}\left(Z_{n}\right)
\end{array}\right) .
\end{aligned}
$$

It follows that

$$
\begin{aligned}
& \frac{1}{K_{n}^{2}}\left|\sum_{1 \leq i<j<k \leq n} f_{j, n} f_{k, n} g_{i j, n} g_{i k, n} \sigma_{\eta \eta, i}\left(Z_{n}\right) \sigma_{u u, j}\left(Z_{n}\right) \sigma_{u u, k}\left(Z_{n}\right)\right| \\
\leq & \frac{1}{2 K_{n}^{2}} f_{n}^{\prime} \Omega_{u u, n}\left(Z_{n}\right) H_{1 n}\left(P_{Z_{n}}^{U}\right)^{\prime} \Omega_{\eta \eta, n}\left(Z_{n}\right) P_{Z_{n}}^{U} H_{1 n} \Omega_{u u, n}\left(Z_{n}\right) f_{n}+ \\
& \frac{1}{2 K_{n}^{2}} \sum_{1 \leq i<j \leq n} f_{j, n}^{2} g_{i j, n}^{2} \sigma_{\eta \eta, i}\left(Z_{n}\right) \sigma_{u u, j}^{2}\left(Z_{n}\right)
\end{aligned}
$$


Next, note that, with probability one for $n$ sufficiently large, we have

$$
\begin{aligned}
& K_{n}^{-2} f_{n}^{\prime} \Omega_{u u, n}\left(Z_{n}\right) H_{1 n}\left(P_{Z_{n}}^{U}\right)^{\prime} \Omega_{\eta \eta, n}\left(Z_{n}\right) P_{Z_{n}}^{U} H_{1 n} \Omega_{u u, n}\left(Z_{n}\right) f_{n} \\
\leq & K_{n}^{-2} \bar{D}_{\xi} f_{n}^{\prime} \Omega_{u u, n}\left(Z_{n}\right) H_{1 n}\left(P_{Z_{n}}^{U}\right)^{\prime} P_{Z_{n}}^{U} H_{1 n} \Omega_{u u, n}\left(Z_{n}\right) f_{n} \\
\leq & K_{n}^{-2} \lambda_{\max }\left(\left(P_{Z_{n}}^{U}\right)^{\prime} P_{Z_{n}}^{U}\right) \bar{D}_{\xi} f_{n}^{\prime} \Omega_{u u, n}\left(Z_{n}\right) H_{1 n}^{2} \Omega_{u u, n}\left(Z_{n}\right) f_{n} \\
\leq & K_{n}^{-2}\left\|\left(P_{Z_{n}}^{U}\right)^{\prime} P_{Z_{n}}^{U}\right\|_{F} \bar{D}_{\xi} f_{n}^{\prime} \Omega_{u u, n}\left(Z_{n}\right) H_{1 n}^{2} \Omega_{u u, n}\left(Z_{n}\right) f_{n} \\
\leq & K_{n}^{-2}\left\|\left(P_{Z_{n}}^{U}\right)^{\prime} P_{Z_{n}}^{U}\right\|_{F}\left(\frac{1}{1-\bar{h}}\right)^{2} \bar{D}_{\xi} f_{n}^{\prime}\left[\Omega_{u u, n}\left(Z_{n}\right)\right]^{2} f_{n} \\
\leq & K_{n}^{-1}\left\|\left(P_{Z_{n}}^{U}\right)^{\prime} P_{Z_{n}}^{U}\right\|_{F}\left(\frac{1}{1-\bar{h}}\right)^{2} \bar{D}_{\xi}^{3}\left(\frac{f_{n}^{\prime} f_{n}}{K_{n}}\right) \\
= & O_{a . s .}\left(K_{n}^{-\frac{1}{2}}\right),
\end{aligned}
$$

where the first and the fifth inequalities follow from Assumption 3, the second inequality follow from applying the spectral decomposition of the positive semidefinite matrix $\left(P_{Z_{n}}^{U}\right)^{\prime} P_{Z_{n}}^{U}$, the third inequality follows from the fact that $\lambda_{\max }\left(\left(P_{Z_{n}}^{U}\right)^{\prime} P_{Z_{n}}^{U}\right) \leq\left\|\left(P_{Z_{n}}^{U}\right)^{\prime} P_{Z_{n}}^{U}\right\|_{F}$, as shown in Theorem 5.6.9 on page 297 of Horn and Johnson (1985), the fourth inequality follows from the fact that, with probability one, the diagonal elements of $H_{1 n}^{2}$ are bounded uniformly from above by $\left(\frac{1}{1-\bar{h}}\right)^{2}$ in light of Assumption, and the last equality follows from Lemma A3. Moreover, with probability one for $n$ sufficiently large, $\frac{1}{K_{n}^{2}} \sum_{1 \leq i<j \leq n} f_{j, n}^{2} g_{i j, n}^{2} \sigma_{\eta \eta, i}\left(Z_{n}\right) \sigma_{u u, j}^{2}\left(Z_{n}\right) \leq \bar{D}_{\xi \frac{1}{K_{n}^{2}}}^{3} \sum_{1 \leq i<j \leq n} f_{j, n}^{2} g_{i j, n}^{2}$ given Assumption, so that

$$
\frac{1}{K_{n}^{2}} \sum_{1 \leq i<j \leq n} f_{j, n}^{2} g_{i j, n}^{2} \sigma_{\eta \eta, i}\left(Z_{n}\right) \sigma_{u u, j}^{2}\left(Z_{n}\right)=o_{a . s .}(1)
$$

in light of part (b) of this lemma. The desired result follows immediately from (28), (29), and (30).

Parts (f)-(h) can be proved in a manner similar to the proof of part (d) above. Hence, for the sake of brevity, we omit these proofs.

\section{Lemma A7:}

Let $G_{n}$ and $g_{i j, n}$ be as defined in Lemma A4. Then, under Assumptions 2 and 6, the folowing statements are true as $n \rightarrow \infty$

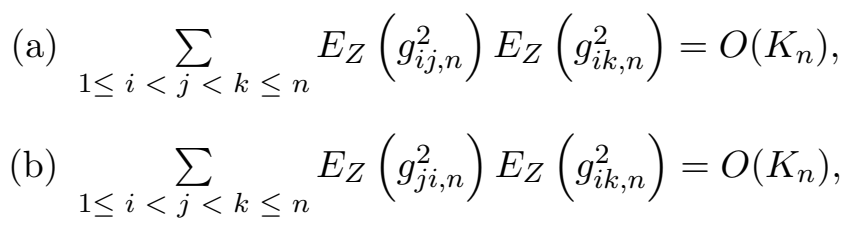


(c) $\sum_{1 \leq i<j<k \leq n} E_{Z}\left(g_{i j, n}^{2}\right) E_{Z}\left(g_{k i, n}^{2}\right)=O\left(K_{n}\right)$,

(d) $\sum_{1 \leq i<j<k \leq n} E_{Z}\left(g_{j i, n}^{2}\right) E_{Z}\left(g_{k i, n}^{2}\right)=O\left(K_{n}\right)$,

\section{Proof of Lemma A7:}

To prove part (a), note that for $n$ sufficiently large so that the inverse $\left(Z_{n}^{\prime} Z_{n}\right)^{-1}$ is well-defined with probability one given Assumption 2, we have

$$
\begin{aligned}
K_{n} & =E_{Z}\left[\operatorname{Tr}\left(P_{Z_{n}}^{4}\right)\right] \\
& \geq E_{Z}\left[\sum_{1 \leq i \leq n}\left(\sum_{1 \leq j \leq n}\left(p_{i j, n}^{Z}\right)^{2}\right)^{2}\right] \\
& \geq \sum_{i=1}^{n}\left(\sum_{j=1}^{n} E_{Z}\left[\left(p_{i j, n}^{Z}\right)^{2}\right]\right)^{2} \\
& \geq \sum_{1 \leq i<j \leq n}\left(E_{Z}\left[\left(p_{i j, n}^{Z}\right)^{2}\right]\right)^{2}+\sum_{1 \leq i<j<k \leq n} E_{Z}\left[\left(p_{i j, n}^{Z}\right)^{2}\right] E\left[\left(p_{i k, n}^{Z}\right)^{2}\right],
\end{aligned}
$$

where the second inequality above follows from application of the Jensen's inequality. Since $\sum_{1 \leq i<j \leq n}\left(E_{Z}\left[\left(p_{i j, n}^{Z}\right)^{2}\right]\right)^{2}$ and $\sum_{1 \leq i<j<k \leq n} E_{Z}\left[\left(p_{i j, n}^{Z}\right)^{2}\right] E_{Z}\left[\left(p_{i k, n}^{Z}\right)^{2}\right]$ are both non-negative, it follows that $\sum_{1 \leq i<j<k \leq n} E_{Z}\left[\left(p_{i j, n}^{Z}\right)^{2}\right] E_{Z}\left[\left(p_{i k, n}^{Z}\right)^{2}\right]=O\left(K_{n}\right)$. Now, note that

$$
\begin{aligned}
\sum_{1 \leq i<j<k \leq n} E_{Z}\left(g_{i j, n}^{2}\right) E_{Z}\left(g_{i k, n}^{2}\right) & =\sum_{1 \leq i<j<k \leq n} E_{Z}\left[\frac{\left(p_{i j, n}^{Z}\right)^{2}}{\left(1-p_{j j, n}^{Z}\right)^{2}}\right] E_{Z}\left[\frac{\left(p_{i k, n}^{Z}\right)^{2}}{\left(1-p_{k k, n}^{Z}\right)^{2}}\right] \\
& \leq\left(\frac{1}{1-\bar{h}}\right)^{4} \sum_{1 \leq i<j<k \leq n} E_{Z}\left[\left(p_{i j, n}^{Z}\right)^{2}\right] E_{Z}\left[\left(p_{i k, n}^{Z}\right)^{2}\right] \\
& =O\left(K_{n}\right) .
\end{aligned}
$$

Parts (b)-(d) can be proved in a similar manner. For sake of brevity, we omit these proofs.

Lemma A8: Define $G_{n}, f_{n}$, and $\eta_{n}$ be as defined in (17), (20), and (21), respectively. Consider the linear-bilinear form

$$
W_{n}=f_{n}^{\prime} u_{n}+\eta_{n}^{\prime} G_{n} u_{n}
$$


Let $\sigma_{W_{n}}^{2}$ denote the variance of $W_{n}$. Suppose that Assumptions hold and suppose that $\frac{r_{n}}{K_{n}} \rightarrow \kappa$ for $0 \leq \kappa<\infty$ but $\frac{\sqrt{K_{n}}}{r_{n}} \rightarrow 0$ as $n \rightarrow \infty$. Then, $\sigma_{W_{n}}^{2}=K_{n}$

\section{Proof of Lemma A8:}

To proceed, note that we can write $W_{n}=\sum_{j=1}^{n} W_{j n}$, where

$$
W_{j n}=f_{j, n} u_{j}+\sum_{1 \leq i<j}\left(g_{i j, n} \eta_{i, n} u_{j}+g_{j i, n} \eta_{j, n} u_{i}\right)
$$

Moreover, since the sequence $\left\{\epsilon_{i}\right\}$ (where $\epsilon_{i}=\left(u_{i}, \eta_{i}\right)$ ) is conditionally independent given $Z_{n}$ by Assumption 3, it is easy to see that $\sigma_{W_{n}}^{2}=\sum_{j=1}^{n} E\left(W_{j n}^{2}\right)$. It follows by straightforward calculation that

$$
\begin{aligned}
\sigma_{W_{n}}^{2}= & \sum_{j=1}^{n} E_{Z}\left[f_{j, n}^{2} \sigma_{u u, j}\left(Z_{n}\right)\right]+\sum_{1 \leq i<j \leq n} E_{Z}\left[g_{i j, n}^{2} \sigma_{\eta \eta, i}\left(Z_{n}\right) \sigma_{u u, j}\left(Z_{n}\right) \mid\right. \\
& \left.+2 g_{i j, n} g_{j i, n} \sigma_{\eta u, i}\left(Z_{n}\right) \sigma_{\eta u, j}\left(Z_{n}\right)+g_{j i, n}^{2} \sigma_{\eta \eta, j}\left(Z_{n}\right) \sigma_{u u, i}\left(Z_{n}\right)\right]
\end{aligned}
$$

Next, note that

$$
\begin{aligned}
\bar{\Xi}_{n}= & \frac{1}{K_{n}} \sum_{j=1}^{n} b_{n}^{-2} C_{n}^{\prime} Z_{n, j} Z_{n, i}^{\prime} C_{n} \sigma_{u u, j}\left(Z_{n}\right) \\
& +\frac{1}{K_{n}} \sum_{1 \leq i<j \leq n}\left[g_{i j, n}^{2} \Sigma_{V V, i}\left(Z_{n}\right) \sigma_{u u, j}\left(Z_{n}\right)+g_{i j, n} g_{j i, n} \sigma_{V u, i}\left(Z_{n}\right) \sigma_{V u, j}^{\prime}\left(Z_{n}\right)\right. \\
& \left.+g_{i j, n} g_{j i, n} \sigma_{V u, j}\left(Z_{n}\right) \sigma_{V u, i}^{\prime}\left(Z_{n}\right)+g_{j i, n}^{2} \Sigma_{V V, j}\left(Z_{n}\right) \sigma_{u u, i}\left(Z_{n}\right)\right] . \\
\sigma_{\eta \eta, i}\left(Z_{n}\right)= & \gamma^{\prime}\left(\bar{\Psi}_{n}^{-1} \bar{\Xi}_{n} \bar{\Psi}_{n}^{-1}\right)^{-\frac{1}{2}} \bar{\Psi}_{n}^{-1} \Sigma_{V V, i}\left(Z_{n}\right) \bar{\Psi}_{n}^{-1}\left(\bar{\Psi}_{n}^{-1} \bar{\Xi}_{n} \bar{\Psi}_{n}^{-1}\right)^{-\frac{1}{2}} \gamma \\
\sigma_{\eta \eta, i}\left(Z_{n}\right)= & \gamma^{\prime}\left(\bar{\Psi}_{n}^{-1} \bar{\Xi}_{n} \bar{\Psi}_{n}^{-1}\right)^{-\frac{1}{2}} \bar{\Psi}_{n}^{-1} \sigma_{V u, i}\left(Z_{n}\right)
\end{aligned}
$$


It follows that by direct calculation that

$$
\begin{aligned}
\frac{\sigma_{W_{n}}^{2}}{K_{n}}= & \frac{1}{K_{n}} \sum_{j=1}^{n} E_{Z}\left\{\gamma^{\prime}\left(\bar{\Psi}_{n}^{-1} \bar{\Xi}_{n} \bar{\Psi}_{n}^{-1}\right)^{-\frac{1}{2}} \bar{\Psi}_{n}^{-1}\left[\frac{C_{n}^{\prime} Z_{n, j} Z_{n, i}^{\prime} C_{n} \sigma_{u u, j}\left(Z_{n}\right)}{b_{n}^{2}}\right]\right. \\
& \left.\times \bar{\Psi}_{n}^{-1}\left(\bar{\Psi}_{n}^{-1} \bar{\Xi}_{n} \bar{\Psi}_{n}^{-1}\right)^{-\frac{1}{2}} \gamma\right\} \\
& +\frac{1}{K_{n}} \sum_{1 \leq i<j \leq n} E_{Z}\left\{\gamma ^ { \prime } ( \overline { \Psi } _ { n } ^ { - 1 } \overline { \Xi } _ { n } \overline { \Psi } _ { n } ^ { - 1 } ) ^ { - \frac { 1 } { 2 } } \overline { \Psi } _ { n } ^ { - 1 } \left[g_{i j, n}^{2} \Sigma_{V V, i}\left(Z_{n}\right) \sigma_{u u, j}\left(Z_{n}\right)\right.\right. \\
& +g_{i j, n} g_{j i, n} \sigma_{V u, i}\left(Z_{n}\right) \sigma_{V u, j}^{\prime}\left(Z_{n}\right)+g_{i j, n} g_{j i, n} \sigma_{V u, j}\left(Z_{n}\right) \sigma_{V u, i}^{\prime}\left(Z_{n}\right) \\
& \left.\left.+g_{j i, n}^{2} \Sigma_{V V, j}\left(Z_{n}\right) \sigma_{u u, i}\left(Z_{n}\right)\right] \bar{\Psi}_{n}^{-1}\left(\bar{\Psi}_{n}^{-1} \bar{\Xi}_{n} \bar{\Psi}_{n}^{-1}\right)^{-\frac{1}{2}} \gamma\right\} \\
= & E_{Z}\left[\gamma^{\prime}\left(\bar{\Psi}_{n}^{-1} \bar{\Xi}_{n} \bar{\Psi}_{n}^{-1}\right)^{-\frac{1}{2}} \bar{\Psi}_{n}^{-1} \bar{\Xi}_{n} \bar{\Psi}_{n}^{-1}\left(\bar{\Psi}_{n}^{-1} \bar{\Xi}_{n} \bar{\Psi}_{n}^{-1}\right)^{-\frac{1}{2}} \gamma\right] \\
= & 1 . \square
\end{aligned}
$$

Lemma A9: Let $_{n}$ be as defined in (17) above and let $g_{i j, n}$ denote the $(i, j)^{\text {th }}$ off-diagonal element of the matrix $G_{n}$. Then, under Assumption, as $n \rightarrow \infty$,

$$
\frac{1}{K_{n}^{2}} \mathcal{T}=o_{a . s .}(1)
$$


where $T=T_{1}+T_{2}+T_{3}$, with

$$
\begin{aligned}
\mathcal{T}_{1}= & \sum_{1 \leq i<j<k<l \leq n} g_{i l, n} g_{l j, n} g_{j k, n} g_{k i, n} \sigma_{\eta u, i}\left(Z_{n}\right) \sigma_{\eta u, j}\left(Z_{n}\right) \sigma_{\eta u, k}\left(Z_{n}\right) \sigma_{\eta u, l}\left(Z_{n}\right) \\
& +g_{i k, n} g_{k j, n} g_{j l, n} g_{l i, n} \sigma_{\eta u, i}\left(Z_{n}\right) \sigma_{\eta u, j}\left(Z_{n}\right) \sigma_{\eta u, l}\left(Z_{n}\right) \sigma_{\eta u, k}\left(Z_{n}\right) \\
& +g_{k j, n} g_{k i, n} g_{l i, n} g_{l j, n} \sigma_{u u, j}\left(Z_{n}\right) \sigma_{u u, i}\left(Z_{n}\right) \sigma_{\eta \eta, l}\left(Z_{n}\right) \sigma_{\eta \eta, k}\left(Z_{n}\right) \\
& +g_{i k, n} g_{i l, n} g_{j l, n} g_{j k, n} \sigma_{u u, k}\left(Z_{n}\right) \sigma_{u u, l}\left(Z_{n}\right) \sigma_{\eta \eta, j}\left(Z_{n}\right) \sigma_{\eta \eta, i}\left(Z_{n}\right) \\
& +g_{l i, n} g_{l j, n} g_{j k, n} g_{i k, n} \sigma_{\eta u, i}\left(Z_{n}\right) \sigma_{\eta u, j}\left(Z_{n}\right) \sigma_{u u, k}\left(Z_{n}\right) \sigma_{\eta \eta, l}\left(Z_{n}\right) \\
& +g_{k i, n} g_{k j, n} g_{j l, n} g_{i l, n} \sigma_{\eta u, i}\left(Z_{n}\right) \sigma_{\eta u, j}\left(Z_{n}\right) \sigma_{u u, l}\left(Z_{n}\right) \sigma_{\eta \eta, k}\left(Z_{n}\right) \\
& +g_{j k, n} g_{j l, n} g_{l i, n} g_{k i, n} \sigma_{\eta u, k}\left(Z_{n}\right) \sigma_{\eta u, l}\left(Z_{n}\right) \sigma_{u u, i}\left(Z_{n}\right) \sigma_{\eta \eta, j}\left(Z_{n}\right) \\
& +g_{i k, n} g_{i l, n} g_{l j, n} g_{k j, n} \sigma_{\eta u, k}\left(Z_{n}\right) \sigma_{\eta u, l}\left(Z_{n}\right) \sigma_{u u, j}\left(Z_{n}\right) \sigma_{\eta \eta, i}\left(Z_{n}\right) \\
& +g_{l i, n} g_{l j, n} g_{j k, n} g_{k i, n} \sigma_{u u, i}\left(Z_{n}\right) \sigma_{\eta u, j}\left(Z_{n}\right) \sigma_{\eta u, k}\left(Z_{n}\right) \sigma_{\eta \eta, l}\left(Z_{n}\right) \\
& +g_{k i, n} g_{k j, n} g_{j l, n} g_{l i, n} \sigma_{u u, i}\left(Z_{n}\right) \sigma_{\eta u, j}\left(Z_{n}\right) \sigma_{\eta u, l}\left(Z_{n}\right) \sigma_{\eta \eta, k}\left(Z_{n}\right) \\
& +g_{l j, n} g_{l i, n} g_{i k, n} g_{k j, n} \sigma_{u u, j}\left(Z_{n}\right) \sigma_{\eta u, i}\left(Z_{n}\right) \sigma_{\eta u, k}\left(Z_{n}\right) \sigma_{\eta \eta, l}\left(Z_{n}\right) \\
& +g_{k j, n} g_{k i, n} g_{i l, n} g_{l j, n} \sigma_{u u, j}\left(Z_{n}\right) \sigma_{\eta u, i}\left(Z_{n}\right) \sigma_{\eta u, l}\left(Z_{n}\right) \sigma_{\eta \eta, k}\left(Z_{n}\right) \\
& +g_{k j, n} g_{k i, n} g_{i l, n} g_{l j, n} \sigma_{u u, j}\left(Z_{n}\right) \sigma_{\eta u, i}\left(Z_{n}\right) \sigma_{\eta u, l}\left(Z_{n}\right) \sigma_{\eta \eta, k}\left(Z_{n}\right) \\
& +g_{i k, n} g_{i l, n} g_{l j, n} g_{j k, n} \sigma_{u u, k}\left(Z_{n}\right) \sigma_{\eta u, l}\left(Z_{n}\right) \sigma_{\eta u, j}\left(Z_{n}\right) \sigma_{\eta \eta, i}\left(Z_{n}\right) \\
& +g_{j l, n} g_{j k, n} g_{k i, n} g_{i l, n} \sigma_{u u, l}\left(Z_{n}\right) \sigma_{\eta u, k}\left(Z_{n}\right) \sigma_{\eta u, i}\left(Z_{n}\right) \sigma_{\eta \eta, j}\left(Z_{n}\right) \\
& +g_{i l, n} g_{i k, n} g_{k j, n} g_{j l, n} \sigma_{u u, l}\left(Z_{n}\right) \sigma_{\eta u, k}\left(Z_{n}\right) \sigma_{\eta u, j}\left(Z_{n}\right) \sigma_{\eta \eta, i}\left(Z_{n}\right)
\end{aligned}
$$




$$
\begin{aligned}
\mathcal{T}_{2}= & \sum_{1 \leq i<j<k<l \leq n} g_{i l, n} g_{l k, n} g_{k j, n} g_{j i, n} \sigma_{\eta u, i}\left(Z_{n}\right) \sigma_{\eta u, k}\left(Z_{n}\right) \sigma_{\eta u, j}\left(Z_{n}\right) \sigma_{\eta u, l}\left(Z_{n}\right) \\
& +g_{l i, n} g_{l k, n} g_{j k, n} g_{j i, n} \sigma_{u u, i}\left(Z_{n}\right) \sigma_{u u, k}\left(Z_{n}\right) \sigma_{\eta \eta, j}\left(Z_{n}\right) \sigma_{\eta \eta, l}\left(Z_{n}\right) \\
& +g_{l i, n} g_{l k, n} g_{k j, n} g_{i j, n} \sigma_{\eta u, i}\left(Z_{n}\right) \sigma_{\eta u, k}\left(Z_{n}\right) \sigma_{u u, j}\left(Z_{n}\right) \sigma_{\eta \eta, l}\left(Z_{n}\right) \\
& +g_{l i, n} g_{l k, n} g_{k j, n} g_{j i, n} \sigma_{u u, i}\left(Z_{n}\right) \sigma_{\eta u, k}\left(Z_{n}\right) \sigma_{\eta u, j}\left(Z_{n}\right) \sigma_{\eta \eta, l}\left(Z_{n}\right) \\
& +g_{i j, n} g_{j k, n} g_{k l, n} g_{l i, n} \sigma_{\eta u, i}\left(Z_{n}\right) \sigma_{\eta u, k}\left(Z_{n}\right) \sigma_{\eta u, l}\left(Z_{n}\right) \sigma_{\eta u, j}\left(Z_{n}\right) \\
& +g_{j i, n} g_{j k, n} g_{k l, n} g_{i l, n} \sigma_{\eta u, i}\left(Z_{n}\right) \sigma_{\eta u, k}\left(Z_{n}\right) \sigma_{u u, l}\left(Z_{n}\right) \sigma_{\eta \eta, j}\left(Z_{n}\right) \\
& +g_{j i, n} g_{j k, n} g_{k l, n} g_{l i, n} \sigma_{u u, i}\left(Z_{n}\right) \sigma_{\eta u, k}\left(Z_{n}\right) \sigma_{\eta u, l}\left(Z_{n}\right) \sigma_{\eta \eta, j}\left(Z_{n}\right) \\
& +g_{k j, n} g_{k l, n} g_{i l, n} g_{i j, n} \sigma_{u u, j}\left(Z_{n}\right) \sigma_{u u, l}\left(Z_{n}\right) \sigma_{\eta \eta, i}\left(Z_{n}\right) \sigma_{\eta \eta, k}\left(Z_{n}\right) \\
& +g_{k j, n} g_{k l, n} g_{l i, n} g_{j i, n} \sigma_{\eta u, j}\left(Z_{n}\right) \sigma_{\eta u, l}\left(Z_{n}\right) \sigma_{u u, i}\left(Z_{n}\right) \sigma_{\eta \eta, k}\left(Z_{n}\right) \\
& +g_{k j, n} g_{k l, n} g_{l i, n} g_{j i, n} \sigma_{\eta u, j}\left(Z_{n}\right) \sigma_{\eta u, l}\left(Z_{n}\right) \sigma_{u u, i}\left(Z_{n}\right) \sigma_{\eta \eta, k}\left(Z_{n}\right) \\
& +g_{k j, n} g_{k l, n} g_{l i, n} g_{i j, n} \sigma_{u u, j}\left(Z_{n}\right) \sigma_{\eta u, l}\left(Z_{n}\right) \sigma_{\eta u, i}\left(Z_{n}\right) \sigma_{\eta \eta, k}\left(Z_{n}\right) \\
& +g_{i j, n} g_{i l, n} g_{l k, n} g_{k j, n} \sigma_{u u, j}\left(Z_{n}\right) \sigma_{\eta u, l}\left(Z_{n}\right) \sigma_{\eta u, k}\left(Z_{n}\right) \sigma_{\eta \eta, i}\left(Z_{n}\right) \\
& +g_{l k, n} g_{l i, n} g_{i j, n} g_{j k, n} \sigma_{u u, k}\left(Z_{n}\right) \sigma_{\eta u, i}\left(Z_{n}\right) \sigma_{\eta u, j}\left(Z_{n}\right) \sigma_{\eta \eta, l}\left(Z_{n}\right) \\
& +g_{j k, n} g_{j i, n} g_{i l, n} g_{l k, n} \sigma_{u u, k}\left(Z_{n}\right) \sigma_{\eta u, i}\left(Z_{n}\right) \sigma_{\eta u, l}\left(Z_{n}\right) \sigma_{\eta \eta, j}\left(Z_{n}\right) \\
& +g_{k l, n} g_{k j, n} g_{j i, n} g_{i l, n} \sigma_{u u, l}\left(Z_{n}\right) \sigma_{\eta u, j}\left(Z_{n}\right) \sigma_{\eta u, i}\left(Z_{n}\right) \sigma_{\eta \eta, k}\left(Z_{n}\right) \\
& +g_{i l, n} g_{i j, n} g_{j k, n} g_{k l, n} \sigma_{u u, l}\left(Z_{n}\right) \sigma_{\eta u, j}\left(Z_{n}\right) \sigma_{\eta u, k}\left(Z_{n}\right) \sigma_{\eta \eta, i}\left(Z_{n}\right)
\end{aligned}
$$


and

$$
\begin{aligned}
\mathcal{T}_{3}= & \sum_{1 \leq i<j<k<l \leq n} g_{i k, n} g_{k l, n} g_{l j, n} g_{j i, n} \sigma_{\eta u, i}\left(Z_{n}\right) \sigma_{\eta u, l}\left(Z_{n}\right) \sigma_{\eta u, j}\left(Z_{n}\right) \sigma_{\eta u, k}\left(Z_{n}\right) \\
& +g_{k i, n} g_{k l, n} g_{j l, n} g_{j i, n} \sigma_{u u, i}\left(Z_{n}\right) \sigma_{u u, l}\left(Z_{n}\right) \sigma_{\eta \eta, j}\left(Z_{n}\right) \sigma_{\eta \eta, k}\left(Z_{n}\right) \\
& +g_{k i, n} g_{k l, n} g_{l j, n} g_{i j, n} \sigma_{\eta u, i}\left(Z_{n}\right) \sigma_{\eta u, l}\left(Z_{n}\right) \sigma_{u u, j}\left(Z_{n}\right) \sigma_{\eta \eta, k}\left(Z_{n}\right) \\
& +g_{k i, n} g_{k l, n} g_{l j, n} g_{j i, n} \sigma_{u u, i}\left(Z_{n}\right) \sigma_{\eta u, l}\left(Z_{n}\right) \sigma_{\eta u, j}\left(Z_{n}\right) \sigma_{\eta \eta, k}\left(Z_{n}\right) \\
& +g_{i j, n} g_{j l, n} g_{l k, n} g_{k i, n} \sigma_{\eta u, i}\left(Z_{n}\right) \sigma_{\eta u, l}\left(Z_{n}\right) \sigma_{\eta u, k}\left(Z_{n}\right) \sigma_{\eta u, j}\left(Z_{n}\right) \\
& +g_{j i, n} g_{j l, n} g_{l k, n} g_{i k, n} \sigma_{\eta u, i}\left(Z_{n}\right) \sigma_{\eta u, l}\left(Z_{n}\right) \sigma_{u u, k}\left(Z_{n}\right) \sigma_{\eta \eta, j}\left(Z_{n}\right) \\
& +g_{j i, n} g_{j l, n} g_{l k, n} g_{k i, n} \sigma_{u u, i}\left(Z_{n}\right) \sigma_{\eta u, l}\left(Z_{n}\right) \sigma_{\eta u, k}\left(Z_{n}\right) \sigma_{\eta \eta, j}\left(Z_{n}\right) \\
& +g_{l j, n} g_{l k, n} g_{i k, n} g_{i j, n} \sigma_{u u, j}\left(Z_{n}\right) \sigma_{u u, k}\left(Z_{n}\right) \sigma_{\eta \eta, i}\left(Z_{n}\right) \sigma_{\eta \eta, l}\left(Z_{n}\right) \\
& +g_{l j, n} g_{l k, n} g_{k i, n} g_{j i, n} \sigma_{\eta u, j}\left(Z_{n}\right) \sigma_{\eta u, k}\left(Z_{n}\right) \sigma_{u u, i}\left(Z_{n}\right) \sigma_{\eta \eta, l}\left(Z_{n}\right) \\
& +g_{i j, n} g_{i k, n} g_{k l, n} g_{j l, n} \sigma_{\eta u, j}\left(Z_{n}\right) \sigma_{\eta u, k}\left(Z_{n}\right) \sigma_{u u, l}\left(Z_{n}\right) \sigma_{\eta \eta, i}\left(Z_{n}\right) \\
& +g_{l j, n} g_{l k, n} g_{k i, n} g_{i j, n} \sigma_{u u, j}\left(Z_{n}\right) \sigma_{\eta u, k}\left(Z_{n}\right) \sigma_{\eta u, i}\left(Z_{n}\right) \sigma_{\eta \eta, l}\left(Z_{n}\right) \\
& +g_{i j, n} g_{i k, n} g_{k l, n} g_{l j, n} \sigma_{u u, j}\left(Z_{n}\right) \sigma_{\eta u, k}\left(Z_{n}\right) \sigma_{\eta u, l}\left(Z_{n}\right) \sigma_{\eta \eta, i}\left(Z_{n}\right) \\
& +g_{l k, n} g_{l j, n} g_{j i, n} g_{i k, n} \sigma_{u u, k}\left(Z_{n}\right) \sigma_{\eta u, j}\left(Z_{n}\right) \sigma_{\eta u, i}\left(Z_{n}\right) \sigma_{\eta \eta, l}\left(Z_{n}\right) \\
& +g_{i k, n} g_{i j, n} g_{j l, n} g_{l k, n} \sigma_{u u, k}\left(Z_{n}\right) \sigma_{\eta u, j}\left(Z_{n}\right) \sigma_{\eta u, l}\left(Z_{n}\right) \sigma_{\eta \eta, i}\left(Z_{n}\right) \\
& +g_{k l, n} g_{k i, n} g_{i j, n} g_{j l, n} \sigma_{u u, l}\left(Z_{n}\right) \sigma_{\eta u, i}\left(Z_{n}\right) \sigma_{\eta u, j}\left(Z_{n}\right) \sigma_{\eta \eta, k}\left(Z_{n}\right) \\
& +g_{j l, n} g_{j i, n} g_{i k, n} g_{k l, n} \sigma_{u u, l}\left(Z_{n}\right) \sigma_{\eta u, i}\left(Z_{n}\right) \sigma_{\eta u, k}\left(Z_{n}\right) \sigma_{\eta \eta, j}\left(Z_{n}\right)
\end{aligned}
$$

\section{Proof of Lemma A9:}

To proceed, define $A_{n}=L\left(Z_{n}\right) \bar{G}_{n} L\left(Z_{n}\right)^{\prime}$, where

$$
\begin{aligned}
\bar{G}_{n} & =\left(\begin{array}{cc}
0 & \frac{1}{2} G_{n}^{\prime} \\
\frac{1}{2} G_{n} & 0
\end{array}\right), \\
L\left(Z_{n}\right) & =\left(\begin{array}{cc}
L_{11}\left(Z_{n}\right) & 0 \\
L_{21}\left(Z_{n}\right) & L_{22}\left(Z_{n}\right)
\end{array}\right),
\end{aligned}
$$

$L_{11}\left(Z_{n}\right)=\operatorname{diag}\left(\sigma_{u u \cdot \eta, 1}^{\frac{1}{2}}\left(Z_{n}\right), \ldots . ., \sigma_{u u . \eta, n}^{\frac{1}{2}}\left(Z_{n}\right)\right)$ with $\sigma_{u u . \eta, i}^{\frac{1}{2}}\left(Z_{n}\right)=\left(\sigma_{u u, i}\left(Z_{n}\right)-\sigma_{u \eta, i}^{2}\left(Z_{n}\right) / \sigma_{\eta \eta, i}\left(Z_{n}\right)\right)^{\frac{1}{2}}$ $(i=1, \ldots, n), L_{21}\left(Z_{n}\right)=\operatorname{diag}\left(\sigma_{u \eta, 1}\left(Z_{n}\right) / \sigma_{\eta \eta, 1}^{\frac{1}{2}}\left(Z_{n}\right), \ldots, \sigma_{u \eta, n}\left(Z_{n}\right) / \sigma_{\eta \eta, n}^{\frac{1}{2}}\left(Z_{n}\right)\right)$, and $L_{22}\left(Z_{n}\right)=$ $\operatorname{diag}\left(\sigma_{\eta \eta, 1}^{\frac{1}{2}}\left(Z_{n}\right), \ldots \ldots, \sigma_{\eta \eta, n}^{\frac{1}{2}}\left(Z_{n}\right)\right)$. Now, by straightforward but tedious calculation, we can show that 


$$
\operatorname{Tr}\left(A_{n}^{4}\right)=\frac{1}{2}\left(\mathcal{T}_{1}+\mathcal{T}_{2}+\mathcal{T}_{3}\right)+\frac{1}{8} \mathcal{T}_{4}+\frac{1}{4}\left(\mathcal{T}_{5}+\mathcal{T}_{6}\right),
$$

where $\mathcal{T}_{1}, \mathcal{T}_{2}$, and $\mathcal{T}_{3}$ are defined in expressions (34)-(36) and where

$$
\begin{aligned}
\mathcal{T}_{4}= & \sum_{1 \leq}\left[\begin{array}{l}
\leq<n \\
+
\end{array} g_{i j, n}^{4} \sigma_{u u, j}^{2} g_{j i, n}^{2} \sigma_{\eta u, i}^{2}\left(Z_{n}\right) \sigma_{\eta \eta, i}^{2}\left(Z_{n}\right)+2 g_{i j, n}^{2} g_{j i, n}^{2} \sigma_{\eta u, i}^{2}\left(Z_{n}\right) \sigma_{u u, j}\left(Z_{n}\right) \sigma_{\eta \eta, j}\left(Z_{n}\right)\right. \\
+ & 2 g_{i j, n}^{2} g_{j i, n}^{2} \sigma_{u u, i}\left(Z_{n}\right) \sigma_{\eta \eta, i}\left(Z_{n}\right) \sigma_{\eta u, j}^{2}\left(Z_{n}\right) \\
+ & 4 g_{i j, n} g_{j i, n}^{3} \sigma_{\eta u, i}\left(Z_{n}\right) \sigma_{u u, i}\left(Z_{n}\right) \sigma_{\eta u, j}\left(Z_{n}\right) \sigma_{\eta \eta, j}\left(Z_{n}\right) \\
+ & \left.4 g_{i j, n}^{3} g_{j i, n} \sigma_{\eta u, i}\left(Z_{n}\right) \sigma_{\eta \eta, i}\left(Z_{n}\right) \sigma_{u u, j}\left(Z_{n}\right) \sigma_{u \eta, j}\left(Z_{n}\right)\right] \\
\mathcal{T}_{5}= & \sum\left[g_{i k, n} g_{k j, n} g_{j k, n} g_{k i, n} \sigma_{\eta u, i}\left(Z_{n}\right) \sigma_{\eta u, j}\left(Z_{n}\right) \sigma_{\eta u, k}^{2}\left(Z_{n}\right)\right. \\
& +g_{k i, n}^{2} g_{k j, n}^{2} \sigma_{u u, i}\left(Z_{n}\right) \sigma_{u u, j}\left(Z_{n}\right) \sigma_{\eta \eta, k}^{2}\left(Z_{n}\right) \\
& +2 g_{i k, n} g_{k j, n} g_{j k, n} g_{k i, n} \sigma_{\eta u, i}\left(Z_{n}\right) \sigma_{\eta u, j}\left(Z_{n}\right) \sigma_{u u, k}\left(Z_{n}\right) \sigma_{\eta \eta, k}\left(Z_{n}\right) \\
& +2 g_{k i, n}^{2} g_{k j, n} g_{j k, n} \sigma_{u u, i}\left(Z_{n}\right) \sigma_{\eta u, j}\left(Z_{n}\right) \sigma_{\eta u, k}\left(Z_{n}\right) \sigma_{\eta \eta, k}\left(Z_{n}\right) \\
& +2 g_{k j, n}^{2} g_{k i, n} g_{i k, n} \sigma_{u u, j}\left(Z_{n}\right) \sigma_{\eta u, i}\left(Z_{n}\right) \sigma_{\eta u, k}\left(Z_{n}\right) \sigma_{\eta \eta, k}\left(Z_{n}\right) \\
& +g_{k j, n} g_{j k, n} g_{k i, n} g_{i k, n} \sigma_{\eta u, k}^{2}\left(Z_{n}\right) \sigma_{\eta u, i}\left(Z_{n}\right) \sigma_{\eta u, j}\left(Z_{n}\right) \\
& +g_{j k, n}^{2} g_{i k, n}^{2} \sigma_{u u, k}^{2}\left(Z_{n}\right) \sigma_{\eta \eta, i}\left(Z_{n}\right) \sigma_{\eta \eta, j}\left(Z_{n}\right) \\
& +g_{j k, n}^{2} g_{k i, n}^{2} \sigma_{\eta u, k}^{2}\left(Z_{n}\right) \sigma_{u u, i}\left(Z_{n}\right) \sigma_{\eta \eta, j}\left(Z_{n}\right) \\
& +2 g_{j k, n}^{2} g_{k i, n} g_{i k, n} \sigma_{u u, k}\left(Z_{n}\right) \sigma_{\eta u, i}\left(Z_{n}\right) \sigma_{\eta u, i}\left(Z_{n}\right) \sigma_{\eta \eta, j}\left(Z_{n}\right) \\
& +g_{i k, n}^{2} g_{k j, n}^{2} \sigma_{\eta u, k}^{2}\left(Z_{n}\right) \sigma_{u u, j}\left(Z_{n}\right) \sigma_{\eta \eta, i}\left(Z_{n}\right) \\
& \left.+2 g_{i k, n}^{2} g_{k j, n} g_{j k, n} \sigma_{u u, k}\left(Z_{n}\right) \sigma_{\eta u, k}\left(Z_{n}\right) \sigma_{\eta u, j}\left(Z_{n}\right) \sigma_{\eta \eta, i}\left(Z_{n}\right)\right]
\end{aligned}
$$




$$
\begin{aligned}
& \mathcal{T}_{6}=\frac{1}{2} \sum_{1 \leq i<j<k \leq n}\left[2 g_{i k, n} g_{k i, n} g_{i j, n} g_{j i, n} \sigma_{\eta u, i}^{2}\left(Z_{n}\right) \sigma_{\eta u, j}\left(Z_{n}\right) \sigma_{\eta u, k}\left(Z_{n}\right)\right. \\
& +2 g_{k i, n}^{2} g_{j i, n}^{2} \sigma_{u u, i}^{2}\left(Z_{n}\right) \sigma_{\eta \eta, j}\left(Z_{n}\right) \sigma_{\eta \eta, k}\left(Z_{n}\right) \\
& +2 g_{k i, n}^{2} g_{i j, n}^{2} \sigma_{\eta u, i}^{2}\left(Z_{n}\right) \sigma_{u u, j}\left(Z_{n}\right) \sigma_{\eta \eta, k}\left(Z_{n}\right) \\
& +4 g_{k i, n}^{2} g_{i j, n} g_{j i, n} \sigma_{u u, i}\left(Z_{n}\right) \sigma_{\eta u, i}\left(Z_{n}\right) \sigma_{\eta u, j}\left(Z_{n}\right) \sigma_{\eta \eta, k}\left(Z_{n}\right) \\
& +g_{j k, n} g_{k j, n} g_{j i, n} g_{i j, n} \sigma_{\eta u, j}^{2}\left(Z_{n}\right) \sigma_{\eta u, i}\left(Z_{n}\right) \sigma_{\eta u, k}\left(Z_{n}\right) \\
& +g_{k j, n}^{2} g_{i j, n}^{2} \sigma_{u u, j}^{2}\left(Z_{n}\right) \sigma_{\eta \eta, i}\left(Z_{n}\right) \sigma_{\eta \eta, k}\left(Z_{n}\right) \\
& +2 g_{k j, n}^{2} g_{j i, n}^{2} \sigma_{\eta u, j}^{2}\left(Z_{n}\right) \sigma_{u u, i}\left(Z_{n}\right) \sigma_{\eta \eta, k}\left(Z_{n}\right) \\
& +4 g_{k j, n}^{2} g_{j i, n} g_{i j, n} \sigma_{u u, j}\left(Z_{n}\right) \sigma_{\eta u, j}\left(Z_{n}\right) \sigma_{\eta u, i}\left(Z_{n}\right) \sigma_{\eta \eta, k}\left(Z_{n}\right) \\
& +g_{j i, n} g_{i j, n} g_{j k, n} g_{k j, n} \sigma_{\eta u, j}^{2}\left(Z_{n}\right) \sigma_{\eta u, k}\left(Z_{n}\right) \sigma_{\eta u, i}\left(Z_{n}\right) \\
& +g_{i j, n}^{2} g_{k j, n}^{2} \sigma_{u u, j}^{2}\left(Z_{n}\right) \sigma_{\eta \eta, k}\left(Z_{n}\right) \sigma_{\eta \eta, i}\left(Z_{n}\right) \\
& +2 g_{i j, n}^{2} g_{j k, n}^{2} \sigma_{\eta u, j}^{2}\left(Z_{n}\right) \sigma_{u u, k}\left(Z_{n}\right) \sigma_{\eta \eta, i}\left(Z_{n}\right) \\
& +4 g_{i j, n}^{2} g_{j k, n} g_{k j, n} \sigma_{u u, j}\left(Z_{n}\right) \sigma_{\eta u, j}\left(Z_{n}\right) \sigma_{\eta u, k}\left(Z_{n}\right) \sigma_{\eta \eta, i}\left(Z_{n}\right) \\
& +2 g_{j i, n}^{2} g_{i k, n}^{2} \sigma_{\eta u, i}^{2}\left(Z_{n}\right) \sigma_{u u, k}\left(Z_{n}\right) \sigma_{\eta \eta, j}\left(Z_{n}\right) \\
& +4 g_{j i, n}^{2} g_{i k, n} g_{k i, n} \sigma_{u u, i}\left(Z_{n}\right) \sigma_{\eta u, i}\left(Z_{n}\right) \sigma_{\eta u, k}\left(Z_{n}\right) \sigma_{\eta \eta, j}\left(Z_{n}\right) \\
& +2 g_{k j, n} g_{j i, n} g_{i j, n} g_{j k, n} \sigma_{\eta u, k}\left(Z_{n}\right) \sigma_{\eta u, i}\left(Z_{n}\right) \sigma_{\eta u, j}^{2}\left(Z_{n}\right) \\
& +2 g_{j k, n}^{2} g_{j i, n}^{2} \sigma_{u u, k}\left(Z_{n}\right) \sigma_{u u, i}\left(Z_{n}\right) \sigma_{\eta \eta, j}^{2}\left(Z_{n}\right) \\
& +4 g_{k j, n} g_{j i, n} g_{i j, n} g_{j k, n} \sigma_{\eta u, k}\left(Z_{n}\right) \sigma_{\eta u, i}\left(Z_{n}\right) \sigma_{u u, j}\left(Z_{n}\right) \sigma_{\eta \eta, j}\left(Z_{n}\right) \\
& +4 g_{j k, n}^{2} g_{j i, n} g_{i j, n} \sigma_{u u, k}\left(Z_{n}\right) \sigma_{\eta u, i}\left(Z_{n}\right) \sigma_{\eta u, j}\left(Z_{n}\right) \sigma_{\eta \eta, j}\left(Z_{n}\right) \\
& +4 g_{j i, n}^{2} g_{j k, n} g_{k j, n} \sigma_{u u, i}\left(Z_{n}\right) \sigma_{\eta u, k}\left(Z_{n}\right) \sigma_{\eta u, j}\left(Z_{n}\right) \sigma_{\eta \eta, j}\left(Z_{n}\right) \\
& +2 g_{j i, n} g_{i k, n} g_{k i, n} g_{i j, n} \sigma_{\eta u, j}\left(Z_{n}\right) \sigma_{\eta u, k}\left(Z_{n}\right) \sigma_{\eta u, i}^{2}\left(Z_{n}\right) \\
& +2 g_{i j, n}^{2} g_{i k, n}^{2} \sigma_{u u, j}\left(Z_{n}\right) \sigma_{u u, k}\left(Z_{n}\right) \sigma_{\eta \eta, i}^{2}\left(Z_{n}\right) \\
& +4 g_{j i, n} g_{i k, n} g_{k i, n} g_{i j, n} \sigma_{\eta u, j}\left(Z_{n}\right) \sigma_{\eta u, k}\left(Z_{n}\right) \sigma_{u u, i}\left(Z_{n}\right) \sigma_{\eta \eta, i}\left(Z_{n}\right) \\
& +4 g_{i j, n}^{2} g_{i k, n} g_{k i, n} \sigma_{u u, j}\left(Z_{n}\right) \sigma_{\eta u, k}\left(Z_{n}\right) \sigma_{\eta u, i}\left(Z_{n}\right) \sigma_{\eta \eta, i}\left(Z_{n}\right) \\
& \left.+4 g_{i k, n}^{2} g_{i j, n} g_{j i, n} \sigma_{u u, k}\left(Z_{n}\right) \sigma_{\eta u, j}\left(Z_{n}\right) \sigma_{\eta u, i}\left(Z_{n}\right) \sigma_{\eta \eta, i}\left(Z_{n}\right)\right]
\end{aligned}
$$

Moreover, let $\lambda_{1}, \ldots, \lambda_{2 n}$ be the eigevalues of the matrix $A_{n}$, and note that $\operatorname{Tr}\left(A_{n}^{4}\right)=\sum_{i=1}^{n} \lambda_{i}^{4}$. Next, 
observe that Lemma A5 implies that

$$
\begin{aligned}
\frac{1}{K_{n}^{2}} \mathcal{T}_{4} \leq & \frac{1}{K_{n}^{2}} \bar{D}_{\xi}^{2} \sum_{1 \leq i<j \leq n}\left[3 g_{i j, n}^{4}+3 g_{j i, n}^{4}+10 g_{i j, n}^{2} g_{j i, n}^{2}\right]=o_{a . s .}(1) \\
\frac{1}{K_{n}^{2}} \mathcal{T}_{5} \leq & \frac{\bar{D}_{\xi}^{2}}{K_{n}^{2}} \sum_{1 \leq i<j<k \leq n}\left[\frac{3}{2} g_{i k, n}^{2} g_{j k, n}^{2}+\frac{5}{2} g_{i j, n}^{2} g_{j k, n}^{2}+3 g_{i j, n}^{2} g_{i k, n}^{2}+\frac{9}{2} g_{k i, n}^{2} g_{k j, n}^{2}\right. \\
& +\frac{5}{2} g_{j i, n}^{2} g_{k j, n}^{2}+\frac{5}{2} g_{j i, n}^{2} g_{i k, n}^{2}+\frac{5}{2} g_{k i, n}^{2} g_{i j, n}^{2}+3 g_{j k, n}^{2} g_{j i, n}^{2}+\frac{5}{2} g_{j k, n}^{2} g_{j i, n}^{2} \\
& \left.+g_{k i, n}^{2} g_{j k, n}^{2}+g_{i k, n}^{2} g_{k j, n}^{2}\right] \\
= & o_{a . s .}(1), \\
\frac{1}{K_{n}^{2}} \mathcal{T}_{6} \leq & \frac{\bar{D}_{\xi}^{2}}{K_{n}^{2}} \sum_{1 \leq i<j<k \leq n} 6 g_{i k, n}^{2} g_{j k, n}^{2}+5 g_{i j, n}^{2} g_{j k, n}^{2}+g_{i j, n}^{2} g_{i k, n}^{2}+g_{k i, n}^{2} g_{k j, n}^{2} \\
& +5 g_{j i, n}^{2} g_{k j, n}^{2}+4 g_{j i, n}^{2} g_{i k, n}^{2}+7 g_{k i, n}^{2} g_{j i, n}^{2}+6 g_{i j, n}^{2} g_{k j, n}^{2}+4 g_{i j, n}^{2} g_{k i, n}^{2} \\
& \left.+4 g_{k i, n}^{2} g_{j k, n}^{2}+4 g_{i k, n}^{2} g_{k j, n}^{2}\right] \\
= & o_{a . s .}(1) .
\end{aligned}
$$

It follows from equation (37) that showing that $K_{n}^{-2} \mathcal{T}=o_{a . s .}(1)$ is equivalent to showing that

$$
\frac{1}{K_{n}^{2}} \sum_{i=1}^{n} \lambda_{i}^{4}=o_{a . s .}(1) \text {. }
$$

To show (44), we first note that

$$
\max _{1 \leq i \leq n} \lambda_{i}^{4} \leq \sum_{i=1}^{n} \lambda_{i}^{4} \leq \max _{1 \leq i \leq n} \lambda_{i}^{2}\left(\sum_{i=1}^{n} \lambda_{i}^{2}\right)
$$


and that with probability one

$$
\begin{aligned}
\frac{1}{K_{n}} \sum_{i=1}^{n} \lambda_{i}^{2}= & \frac{1}{K_{n}} \operatorname{Tr}\left(A_{n}^{2}\right) \\
= & \frac{1}{2 K_{n}} \sum_{1 \leq i<j \leq n}\left[g_{i j, n}^{2} \sigma_{\eta \eta, i}\left(Z_{n}\right) \sigma_{u u, j}\left(Z_{n}\right)+g_{j i, n}^{2} \sigma_{u u, i}\left(Z_{n}\right) \sigma_{\eta \eta, j}\left(Z_{n}\right)\right. \\
& \left.+2 g_{i j, n} g_{j i, n} \sigma_{\eta u, i}\left(Z_{n}\right) \sigma_{\eta u, j}\left(Z_{n}\right)\right] \\
\leq & \frac{1}{K_{n}} \bar{D}_{\xi} \sum_{1 \leq i<j \leq n}\left(g_{i j, n}^{2}+g_{j i, n}^{2}\right) \\
= & \frac{1}{K_{n}} \bar{D}_{\xi} \operatorname{Tr}\left(G_{n}^{\prime} G_{n}\right) \\
= & \frac{1}{K_{n}} \bar{D}_{\xi} \operatorname{Tr}\left(H_{1 n} P_{Z_{n}} H_{1 n}-H_{2 n} P_{Z_{n}} H_{1 n}-H_{1 n} P_{Z_{n}} H_{2 n}+H_{2 n}^{2}\right) \\
\leq & \frac{1}{K_{n}} \bar{D}_{\xi}\left(\frac{1}{1-\bar{h}}\right)^{2}\left[\sum_{i=1}^{n} p_{i i, n}^{Z}+3 \sum_{i=1}^{n}\left(p_{i i, n}^{Z}\right)^{2}\right] \\
\leq & \frac{4}{K_{n}} \bar{D}_{\xi}\left(\frac{1}{1-\bar{h}}\right)^{2} \sum_{i=1}^{n} p_{i i, n}^{Z} \\
\leq & 4 \bar{D}_{\xi}\left(\frac{1}{1-\bar{h}}\right)^{2},
\end{aligned}
$$

where the second inequality follows from Assumption. In light of (45) and (46), to show (44), we need to show that

$$
\frac{1}{K_{n}} \max _{1 \leq i \leq n} \lambda_{i}^{2}=o_{a . s .}(1)
$$

To show (47), we proceed as follows: for a given sample size $n$, let $x$ be any $2 n \times 1$ vector such that $\|x\|=1$, and partition $x=\left(x_{1}^{\prime}, x_{2}^{\prime}\right)^{\prime}$, so that $x_{1}$ and $x_{2}$ are each an $n \times 1$ vector. Now, consider the quadratic form

$$
\begin{aligned}
x^{\prime} A_{n}^{2} x= & \frac{1}{4}\left[x_{1}^{\prime} L_{11}\left(Z_{n}\right) G_{n}^{\prime} L_{22}\left(Z_{n}\right) L_{21}\left(Z_{n}\right) G_{n}^{\prime} L_{22}\left(Z_{n}\right) x_{2}+x_{1}^{\prime} L_{11}\left(Z_{n}\right) G_{n} L_{22}^{2}\left(Z_{n}\right) G_{n} L_{21}\left(Z_{n}\right) x_{2}\right. \\
& +x_{1}^{\prime} L_{11}\left(Z_{n}\right) G_{n}^{\prime} L_{22}^{2}\left(Z_{n}\right) G_{n} L_{11}\left(Z_{n}\right) x_{1}+x_{2}^{\prime} L_{21}\left(Z_{n}\right) G_{n}^{\prime} L_{22}^{2}\left(Z_{n}\right) G_{n} L_{11}\left(Z_{n}\right) x_{1} \\
& +x_{2}^{\prime} L_{22}\left(Z_{n}\right) G_{n} L_{21}\left(Z_{n}\right) L_{22}\left(Z_{n}\right) G_{n} L_{11}\left(Z_{n}\right) x_{1}+x_{2}^{\prime} L_{22}\left(Z_{n}\right) G_{n} L_{11}^{2}\left(Z_{n}\right) G_{n}^{\prime} L_{22}\left(Z_{n}\right) x_{2} \\
& +x_{2}^{\prime} L_{21}\left(Z_{n}\right) G_{n}^{\prime} L_{22}\left(Z_{n}\right) L_{21}\left(Z_{n}\right) G_{n}^{\prime} L_{22}\left(Z_{n}\right) x_{2}+x_{2}^{\prime} L_{22}\left(Z_{n}\right) G_{n} L_{21}^{2}\left(Z_{n}\right) G_{n}^{\prime} L_{22}\left(Z_{n}\right) x_{2} \\
& \left.+x_{2}^{\prime} L_{21}\left(Z_{n}\right) G_{n}^{\prime} L_{22}^{2}\left(Z_{n}\right) G_{n} L_{21}\left(Z_{n}\right) x_{2}+x_{2}^{\prime} L_{22}\left(Z_{n}\right) G_{n} L_{21}\left(Z_{n}\right) L_{22}\left(Z_{n}\right) G_{n} L_{21}\left(Z_{n}\right) x_{2}\right]
\end{aligned}
$$


Note that, with probability one,

$$
\begin{aligned}
& \left|x_{1}^{\prime} L_{11}\left(Z_{n}\right) G_{n}^{\prime} L_{22}\left(Z_{n}\right) L_{21}\left(Z_{n}\right) G_{n}^{\prime} L_{22}\left(Z_{n}\right) x_{2}\right| \\
\leq & \sqrt{x_{1}^{\prime} L_{11}\left(Z_{n}\right) G_{n}^{\prime} L_{22}^{2}\left(Z_{n}\right) G_{n} L_{11}\left(Z_{n}\right) x_{1}} \sqrt{x_{2}^{\prime} L_{22}\left(Z_{n}\right) G_{n} L_{21}^{2}\left(Z_{n}\right) G_{n}^{\prime} L_{22}\left(Z_{n}\right) x_{2}} \\
\leq & \sqrt{\max _{1 \leq i \leq n} \sigma_{\eta \eta, i}\left(Z_{n}\right)} \sqrt{x_{1}^{\prime} L_{11}\left(Z_{n}\right) G_{n}^{\prime} G_{n} L_{11}\left(Z_{n}\right) x_{1}} \\
& \times \sqrt{\frac{\max _{1 \leq i \leq n} \sigma_{u \eta, i}^{2}\left(Z_{n}\right)}{\min _{1 \leq i \leq n} \sigma_{\eta \eta, i}\left(Z_{n}\right)} \sqrt{x_{2}^{\prime} L_{22}\left(Z_{n}\right) G_{n} G_{n}^{\prime} L_{22}\left(Z_{n}\right) x_{2}}} \\
\leq & 4\left(\frac{1}{1-\bar{h}}\right)^{2} \frac{\left(\bar{D}_{\xi}\right)^{\frac{3}{4}}}{\underline{D}_{\xi}^{\frac{1}{2}}} \sqrt{x_{1}^{\prime} L_{11}^{2}\left(Z_{n}\right) x_{1}} \sqrt{x_{2}^{\prime} L_{22}^{2}\left(Z_{n}\right) x_{2}} \\
\leq & 4\left(\frac{1}{1-\bar{h}}\right)^{2} \frac{\left(\bar{D}_{\xi}\right)^{\frac{5}{4}}}{\underline{D}_{\xi}^{\frac{1}{2}}} \\
< & \infty,
\end{aligned}
$$

where the third inequality above follows from the fact that, with probability one,

$$
\begin{aligned}
\lambda_{\max }\left(G_{n}^{\prime} G_{n}\right) & =\lambda_{\max }\left(G_{n} G_{n}^{\prime}\right) \\
& =\lambda_{\max }\left(H_{1 n} P_{Z_{n}} H_{1 n}-H_{2 n} P_{Z_{n}} H_{1 n}-H_{1 n} P_{Z_{n}} H_{2 n}+H_{2 n}^{2}\right) \\
& \leq 4\left(\frac{1}{1-\bar{h}}\right)^{2} .
\end{aligned}
$$

By similar arguments, we can show that, with probability one, $\left|x_{1}^{\prime} L_{11}\left(Z_{n}\right) G_{n} L_{22}^{2}\left(Z_{n}\right) G_{n} L_{21}\left(Z_{n}\right) x_{2}\right| \leq$ $4(1-\bar{h})^{-2}\left(\bar{D}_{\xi}\right)^{\frac{5}{4}} \underline{D}_{\xi}^{-\frac{1}{2}}<\infty$,

$\left|x_{1}^{\prime} L_{11}\left(Z_{n}\right) G_{n} L_{22}^{2}\left(Z_{n}\right) G_{n} L_{11}\left(Z_{n}\right) x_{1}\right| \leq 4(1-\bar{h})^{-2} \bar{D}_{\xi}<\infty,\left|x_{2}^{\prime} L_{22}\left(Z_{n}\right) G_{n} L_{11}^{2}\left(Z_{n}\right) G_{n} L_{22}\left(Z_{n}\right) x_{2}\right| \leq$ $4(1-\bar{h})^{-2} \bar{D}_{\xi}<\infty,\left|x_{2}^{\prime} L_{21}\left(Z_{n}\right) G_{n} L_{22}\left(Z_{n}\right) L_{21}\left(Z_{n}\right) G_{n} L_{22}\left(Z_{n}\right) x_{2}\right| \leq 4(1-\bar{h})^{-2}\left(\bar{D}_{\xi}\right)^{\frac{3}{2}}\left(\underline{D}_{\xi}\right)^{-1}<$ $\infty,\left|x_{2}^{\prime} L_{22}\left(Z_{n}\right) G_{n} L_{21}^{2}\left(Z_{n}\right) G_{n} L_{22}\left(Z_{n}\right) x_{2}\right| \leq 4(1-\bar{h})^{-2}\left(\bar{D}_{\xi}\right)^{\frac{3}{2}}\left(\underline{D}_{\xi}\right)^{-1}<\infty$, and $\left|x_{2}^{\prime} L_{21}\left(Z_{n}\right) G_{n} L_{22}^{2}\left(Z_{n}\right) G_{n} L_{21}\left(Z_{n}\right) x_{2}\right| \leq 4(1-\bar{h})^{-2}\left(\bar{D}_{\xi}\right)^{\frac{3}{2}}\left(\underline{D}_{\xi}\right)^{-1}<\infty$. It follows that, with 
probability one,

$$
\begin{aligned}
x^{\prime} A_{n}^{2} x \leq & \frac{1}{4}\left[4(1-\bar{h})^{-2}\left(\bar{D}_{\xi}\right)^{\frac{5}{4}} \underline{D}_{\xi}^{-\frac{1}{2}}+4(1-\bar{h})^{-2}\left(\bar{D}_{\xi}\right)^{\frac{5}{4}} \underline{D}_{\xi}^{-\frac{1}{2}}+4(1-\bar{h})^{-2} \bar{D}_{\xi}\right. \\
& +4(1-\bar{h})^{-2}\left(\bar{D}_{\xi}\right)^{\frac{5}{4}} \underline{D}_{\xi}^{-\frac{1}{2}}+4(1-\bar{h})^{-2}\left(\bar{D}_{\xi}\right)^{\frac{5}{4}} \underline{D}_{\xi}^{-\frac{1}{2}}+4(1-\bar{h})^{-2} \bar{D}_{\xi} \\
& +4(1-\bar{h})^{-2}\left(\bar{D}_{\xi}\right)^{\frac{3}{2}}\left(\underline{D}_{\xi}\right)^{-1}+4(1-\bar{h})^{-2}\left(\bar{D}_{\xi}\right)^{\frac{3}{2}}\left(\underline{D}_{\xi}\right)^{-1} \\
& \left.+4(1-\bar{h})^{-2}\left(\bar{D}_{\xi}\right)^{\frac{3}{2}}\left(\underline{D}_{\xi}\right)^{-1}+4(1-\bar{h})^{-2}\left(\bar{D}_{\xi}\right)^{\frac{3}{2}}\left(\underline{D}_{\xi}\right)^{-1}\right] \\
= & 2(1-\bar{h})^{-2}\left[2\left(\bar{D}_{\xi}\right)^{\frac{5}{4}} \underline{D}_{\xi}^{-\frac{1}{2}}+\bar{D}_{\xi}+2\left(\bar{D}_{\xi}\right)^{\frac{3}{2}}\left(\underline{D}_{\xi}\right)^{-1}\right] \\
< & \infty .
\end{aligned}
$$

Since

$$
\lambda_{\max }\left(A_{n}^{2}\right)=\max _{x:\|x\|=1} x^{\prime} A_{n}^{2} x,
$$

we deduce that with probability one

$$
\max _{1 \leq i \leq n} \lambda_{i}^{2}=\lambda_{\max }\left(A_{n}^{2}\right) \leq \bar{D}<\infty
$$

where

$$
\bar{D}=2(1-\bar{h})^{-2}\left[2\left(\bar{D}_{\xi}\right)^{\frac{5}{4}} \underline{D}_{\xi}^{-\frac{1}{2}}+\bar{D}_{\xi}+2\left(\bar{D}_{\xi}\right)^{\frac{3}{2}}\left(\underline{D}_{\xi}\right)^{-1}\right]
$$

Note that the upper bound $\bar{D}$ does not depend on $n$ and is a uniform bound on $\max _{1 \leq i \leq n} \lambda_{i}^{2}$. It follows that

$$
\left(\frac{1}{K_{n}}\right) \max _{1 \leq i \leq n} \lambda_{i}^{2}=o_{a . s .}(1)
$$

as required. $\square$

Lemma A10: (Gänsler and Stute, 1977)

Let $\left\{X_{i, n}, \mathcal{F}_{i, n}, 1 \leq i \leq l_{n}, n \geq 1\right\}$ be a square integrable martingale difference array. Also, let $l_{n} \nearrow \infty$ as $n \rightarrow \infty$, and suppose that for all $\varepsilon>0$

$$
\sum_{i=1}^{l_{n}} E\left[X_{i, n}^{2} \mathbf{I}\left(\left|X_{i, n}\right|>\varepsilon\right) \mid \mathcal{F}_{i-1, n}\right] \stackrel{P}{\rightarrow} 0
$$

and

$$
\sum_{i=1}^{l_{n}} E\left[X_{i, n}^{2} \mid \mathcal{F}_{i-1, n}\right] \stackrel{P}{\rightarrow} 1 .
$$


Then, $\sum_{i=1}^{l_{n}} X_{i, n} \stackrel{d}{\rightarrow} N(0,1)$.

Proof of Lemma A10: See Gänsler and Stute (1977).

Remark: Note that, as discussed in Kelejian and Prucha (1999), a sufficient condition for condition (C1) is the following:

\section{Condition C1' :}

$$
\sum_{j=1}^{k_{n}} E\left\{E\left[\left|X_{j, n}\right|^{2+\delta} \mid \mathcal{F}_{j-1, n}\right]\right\} \rightarrow 0
$$

for some $\delta>0$.

Since condition $\mathrm{C1}^{\prime}$ ' is easier to verify in our case, in the proofs which follow, we will be verifying condition $\mathrm{C} 1$ ' instead of condition $\mathrm{C} 1$ for the case $\delta=2$.

Lemma A11: Let $W_{n}$ be as defined in (32) above. Define

$$
B_{n}=\frac{W_{n}}{\sqrt{K_{n}}} .
$$

Suppose that $\frac{r_{n}}{K_{n}} \rightarrow \kappa$ for $0 \leq \kappa<\infty$ but $\frac{\sqrt{K_{n}}}{r_{n}} \rightarrow 0$ as $n \rightarrow \infty$. Then, under Assumptions 1-6,

$$
B_{n} \stackrel{d}{\rightarrow} N(0,1) \quad \text { as } n \rightarrow \infty
$$

\section{Proof of Lemma A11:}

The proof of this lemma involves verifying conditions $\mathrm{C} 1$ ' and $\mathrm{C} 2$ which jointly imply the central limit theorem given in Lemma A10. As discussed in the Remark above, we shall verify conditions C1' in lieu of condition C1. The proof is, thus, divided into two parts: in part I, we check condition C1' and, in part II, we check condition C2.

\section{Checking Condition C1':}

As in the proof of Lemma A8, we can write $W_{n}=\sum_{k=1}^{n} W_{k n}$, where $W_{k n}$ is as defined in (32) above. To verify condition $\mathrm{C} 1$ ' for $\delta=2$, we need to show that $\sum_{k=1}^{n} E\left\{\left(\sigma_{W_{n}}^{-1} W_{k n}\right)^{4}\right\} \rightarrow 0$ as $n \rightarrow \infty$. In light of Lemma A8, this is equivalent to showing that $K_{n}^{-2} \sum_{k=1}^{n} E\left(W_{k n}^{4}\right) \rightarrow 0$ as 
$n \rightarrow \infty$. To proceed, note that direct calculation yields the following expression for the fourth moment of $W_{k n}$

$$
E_{Z}\left(W_{k n}^{4}\right)=\sum_{i=1}^{6} \mathcal{E}_{i k, n}
$$

where

$$
\begin{aligned}
& \mathcal{E}_{1 k, n}=E_{Z}\left(f_{k, n}^{4} E\left(u_{k}^{4} \mid Z_{n}\right)\right) \\
& \mathcal{E}_{2 k, n}=4 E_{Z}\left(\sum _ { 1 \leq i < k } \left[f_{k, n}^{2} g_{i k, n}^{2} E\left(u_{k}^{4} \mid Z_{n}\right) \sigma_{\eta \eta, i}\left(Z_{n}\right)+f_{k, n}^{2} g_{k i, n}^{2} E\left(\eta_{k, n}^{2} u_{k}^{2} \mid Z_{n}\right) \sigma_{u u, i}\left(Z_{n}\right)\right.\right. \\
& \left.\left.+2 f_{k, n}^{2} g_{i k, n} g_{k i, n} E\left(\eta_{k, n} u_{k}^{3} \mid Z_{n}\right) \sigma_{\eta u, i}\left(Z_{n}\right)\right]\right) \\
& \mathcal{E}_{3 k, n}=E_{Z}\left(\sum _ { 1 \leq i < k } \left[g_{i k, n}^{4} E\left(\eta_{i, n}^{4} \mid Z_{n}\right) E\left(u_{k}^{4} \mid Z_{n}\right)+g_{k i, n}^{4} E\left(\eta_{k, n}^{4} \mid Z_{n}\right) E\left(u_{i}^{4} \mid Z_{n}\right)\right.\right. \\
& +6 g_{i k, n}^{2} g_{k i, n}^{2} E\left(u_{i}^{2} \eta_{i, n}^{2} \mid Z_{n}\right) E\left(u_{k}^{2} \eta_{k, n}^{2} \mid Z_{n}\right)+4 g_{i k, n}^{3} g_{k i, n} E\left(u_{i} \eta_{i, n}^{3} \mid Z_{n}\right) E\left(u_{k}^{3} \eta_{k, n} \mid Z_{n}\right) \\
& \left.\left.+4 g_{i k, n} g_{k i, n}^{3} E\left(u_{i}^{3} \eta_{i, n} \mid Z_{n}\right) E\left(u_{k} \eta_{k, n}^{3} \mid Z_{n}\right)\right]\right) \\
& \mathcal{E}_{4 k, n}=4 E_{Z}\left(\sum _ { 1 \leq i < j < k } \left[g_{i k, n}^{2} g_{j k, n}^{2} \sigma_{\eta \eta, i}\left(Z_{n}\right) \sigma_{\eta \eta, j}\left(Z_{n}\right) E\left(u_{k}^{4} \mid Z_{n}\right)\right.\right. \\
& +g_{k i, n}^{2} g_{j k, n}^{2} \sigma_{u u, i}\left(Z_{n}\right) \sigma_{\eta \eta, j}\left(Z_{n}\right) E\left(u_{k}^{2} \eta_{k, n}^{2} \mid Z_{n}\right) \\
& +2 g_{i k, n} g_{k i, n} g_{j k, n}^{2} E\left(u_{k}^{3} \eta_{k, n} \mid Z_{n}\right) \sigma_{\eta u, i}\left(Z_{n}\right) \sigma_{\eta \eta, j}\left(Z_{n}\right) \\
& +2 g_{i k, n}^{2} g_{k j, n} g_{j k, n} E\left(u_{k}^{3} \eta_{k, n} \mid Z_{n}\right) \sigma_{\eta u, j}\left(Z_{n}\right) \sigma_{\eta \eta, i}\left(Z_{n}\right) \\
& \left.\left.+4 g_{i k, n} g_{k i, n} g_{k j, n} g_{j k, n} E\left(u_{k}^{2} \eta_{k, n}^{2} \mid Z_{n}\right) \sigma_{\eta u, i}\left(Z_{n}\right) \sigma_{\eta u, j}\left(Z_{n}\right)\right]\right) \\
& \mathcal{E}_{5 k, n}=4 E_{Z}\left(\sum _ { 1 \leq i < j < k } \left[g_{k i, n}^{2} g_{k j, n}^{2} \sigma_{u u, i}\left(Z_{n}\right) \sigma_{u u, j}\left(Z_{n}\right) E\left(\eta_{k, n}^{4} \mid Z_{n}\right)\right.\right. \\
& +g_{i k, n}^{2} g_{k j, n}^{2} E\left(u_{k}^{2} \eta_{k, n}^{2} \mid Z_{n}\right) \sigma_{\eta \eta, i}\left(Z_{n}\right) \sigma_{u u, j}\left(Z_{n}\right) \\
& +2 g_{k i, n}^{2} g_{k j, n} g_{j k, n} E\left(u_{k} \eta_{k, n}^{3} \mid Z_{n}\right) \sigma_{u u, i}\left(Z_{n}\right) \sigma_{\eta u, j}\left(Z_{n}\right) \\
& \left.\left.+2 g_{i k, n} g_{k i, n} g_{k j, n}^{2} E\left(u_{k} \eta_{k, n}^{3} \mid Z_{n}\right) \sigma_{u u, j}\left(Z_{n}\right) \sigma_{\eta u, i}\left(Z_{n}\right)\right]\right)
\end{aligned}
$$




$$
\begin{gathered}
\mathcal{E}_{6 k, n}=2 E_{Z}\left(\sum _ { 1 \leq i < k } \left[f_{k, n}^{2} g_{i k, n}^{2} E\left(u_{k}^{4} \mid Z_{n}\right) \sigma_{\eta \eta, i}\left(Z_{n}\right)+f_{k, n}^{2} g_{k i, n}^{2} E\left(u_{k}^{2} \eta_{k, n}^{2} \mid Z_{n}\right) \sigma_{u u, i}\left(Z_{n}\right)\right.\right. \\
\left.\left.+f_{k, n}^{2} g_{i k, n} g_{k i, n} E\left(\eta_{k, n} u_{k}^{3} \mid Z_{n}\right) \sigma_{\eta u, i}\left(Z_{n}\right)\right]\right) \\
\mathcal{E}_{7 k, n}=4 E_{Z}\left(\sum _ { 1 \leq i < k } \left[f_{k, n} g_{i k, n}^{3} E\left(\eta_{i, n}^{3} \mid Z_{n}\right) E\left(u_{k}^{4} \mid Z_{n}\right)\right.\right. \\
\left.\left.+f_{k, n} g_{k i, n}^{3} E\left(u_{k} \eta_{k, n}^{3} \mid Z_{n}\right) E\left(u_{i}^{3} \mid Z_{n}\right)\right]\right) \\
\mathcal{E}_{8 k, n}=12 E_{Z}\left(\sum _ { 1 \leq i < k } \left[f_{k, n} g_{i k, n} g_{k i, n}^{2} E\left(u_{k}^{2} \eta_{k, n}^{2} \mid Z_{n}\right) E\left(u_{i}^{2} \eta_{i, n} \mid Z_{n}\right)\right.\right. \\
\left.\left.+f_{k, n} g_{i k, n}^{2} g_{k i, n} E\left(u_{k}^{3} \eta_{k, n} \mid Z_{n}\right) E\left(u_{i} \eta_{i, n}^{2} \mid Z_{n}\right)\right]\right)
\end{gathered}
$$

Now, making use of Lemmas A5 and A6 and Assumption 3, we see that

$$
\begin{aligned}
& \frac{1}{K_{n}^{2}} \sum_{k=1}^{n} \mathcal{E}_{1 k, n} \leq \bar{D}_{\xi} \frac{1}{K_{n}^{2}} \sum_{k=1}^{n} E_{Z}\left(f_{k, n}^{4}\right) \\
& =o(1) \text {, } \\
& \frac{1}{K_{n}^{2}} \sum_{k=1}^{n}\left|\mathcal{E}_{2 k, n}\right| \leq \frac{4}{K_{n}^{2}} E_{Z}\left(\sum _ { 1 \leq i < k \leq n } \left[f_{k, n}^{2} g_{i k, n}^{2} E\left(u_{k}^{4} \mid Z_{n}\right) \sigma_{\eta \eta, i}\left(Z_{n}\right)+\frac{1}{2} f_{k, n}^{2} g_{k i, n}^{2} E\left(\eta_{k, n}^{4} \mid Z_{n}\right) \sigma_{u u, i}\left(Z_{n}\right)\right.\right. \\
& +\frac{1}{2} f_{k, n}^{2} g_{k i, n}^{2} E\left(u_{k}^{4} \mid Z_{n}\right) \sigma_{u u, i}\left(Z_{n}\right)+\frac{1}{2} f_{k, n}^{2} g_{i k, n}^{2} E\left(\eta_{k, n}^{2} u_{k}^{2} \mid Z_{n}\right) \sqrt{\sigma_{u u, i}\left(Z_{n}\right)} \sqrt{\sigma_{\eta \eta, i}\left(Z_{n}\right)} \\
& +\frac{1}{2} f_{k, n}^{2} g_{i k, n}^{2} E\left(u_{k}^{4} \mid Z_{n}\right) \sqrt{\sigma_{u u, i}\left(Z_{n}\right)} \sqrt{\sigma_{\eta \eta, i}\left(Z_{n}\right)} \\
& +\frac{1}{2} f_{k, n}^{2} g_{k i, n}^{2} E\left(\eta_{k, n}^{2} u_{k}^{2} \mid Z_{n}\right) \sqrt{\sigma_{u u, i}\left(Z_{n}\right)} \sqrt{\sigma_{\eta \eta, i}\left(Z_{n}\right)} \\
& \left.\left.+\frac{1}{2} f_{k, n}^{2} g_{k i, n}^{2} E\left(u_{k}^{4} \mid Z_{n}\right) \sqrt{\sigma_{u u, i}\left(Z_{n}\right)} \sqrt{\sigma_{\eta \eta, i}\left(Z_{n}\right)}\right]\right) \\
& \leq \frac{8}{K_{n}^{2}} \bar{D}_{\xi}^{\frac{3}{2}} \sum_{1 \leq i<k \leq n} E_{Z}\left(f_{k, n}^{2} g_{i k, n}^{2}+f_{k, n}^{2} g_{k i, n}^{2}\right) \\
& =o(1) \text {, }
\end{aligned}
$$




$$
\begin{aligned}
\frac{1}{K_{n}^{2}} \sum_{k=1}^{n}\left|\mathcal{E}_{3 k, n}\right| \leq & \frac{1}{K_{n}^{2}} E_{Z}\left(\sum _ { 1 \leq i < k \leq n } \left[g_{i k, n}^{4} E\left(\eta_{i, n}^{4} \mid Z_{n}\right) E\left(u_{k}^{4} \mid Z_{n}\right)+g_{k i, n}^{4} E\left(\eta_{k, n}^{4} \mid Z_{n}\right) E\left(u_{i}^{4} \mid Z_{n}\right)\right.\right. \\
& +6 g_{i k, n}^{2} g_{k i, n}^{2} E\left(u_{i}^{2} \eta_{i, n}^{2} \mid Z_{n}\right) E\left(u_{k}^{2} \eta_{k, n}^{2} \mid Z_{n}\right)+\frac{1}{2} g_{i k, n}^{4} E\left(u_{i}^{2} \eta_{i, n}^{2} \mid Z_{n}\right) E\left(u_{k}^{4} \mid Z_{n}\right) \\
& +\frac{1}{2} g_{i k, n}^{4} E\left(u_{i}^{2} \eta_{i, n}^{2} \mid Z_{n}\right) E\left(u_{k}^{2} \eta_{k, n}^{2} \mid Z_{n}\right)+\frac{1}{2} g_{i k, n}^{4} E\left(\eta_{i, n}^{4} \mid Z_{n}\right) E\left(u_{k}^{4} \mid Z_{n}\right) \\
& +\frac{1}{2} g_{i k, n}^{4} E\left(\eta_{i, n}^{4} \mid Z_{n}\right) E\left(u_{k}^{2} \eta_{k, n}^{2} \mid Z_{n}\right)+\frac{1}{2} g_{i k, n}^{2} g_{k i, n}^{2} E\left(u_{i}^{2} \eta_{i, n}^{2} \mid Z_{n}\right) E\left(u_{k}^{4} \mid Z_{n}\right) \\
& +\frac{1}{2} g_{i k, n}^{2} g_{k i, n}^{2} E\left(u_{i}^{2} \eta_{i, n}^{2} \mid Z_{n}\right) E\left(u_{k}^{2} \eta_{k, n}^{2} \mid Z_{n}\right)+\frac{1}{2} g_{i k, n}^{2} g_{k i, n}^{2}\left(\eta_{i, n}^{4} \mid Z_{n}\right) E\left(u_{k}^{4} \mid Z_{n}\right) \\
& +\frac{1}{2} g_{i k, n}^{2} g_{k i, n}^{2} E\left(\eta_{i, n}^{4} \mid Z_{n}\right) E\left(u_{k}^{2} \eta_{k, n}^{2} \mid Z_{n}\right)+\frac{1}{2} g_{k i, n}^{4} E\left(u_{k}^{2} \eta_{k, n}^{2} \mid Z_{n}\right) E\left(u_{i}^{4} \mid Z_{n}\right) \\
& +\frac{1}{2} g_{k i, n}^{4} E\left(u_{k}^{2} \eta_{k, n}^{2} \mid Z_{n}\right) E\left(u_{i}^{2} \eta_{i, n}^{2} \mid Z_{n}\right)+\frac{1}{2} g_{k i, n}^{4} E\left(\eta_{k, n}^{4} \mid Z_{n}\right) E\left(u_{i}^{4} \mid Z_{n}\right) \\
& +\frac{1}{2} g_{k i, n}^{4} E\left(\eta_{k, n}^{4} \mid Z_{n}\right) E\left(u_{i}^{2} \eta_{i, n}^{2} \mid Z_{n}\right)+\frac{1}{2} g_{i k, n}^{2} g_{k i, n}^{2} E\left(u_{k}^{2} \eta_{k, n}^{2} \mid Z_{n}\right) E\left(u_{i}^{4} \mid Z_{n}\right) \\
& +\frac{1}{2} g_{i k, n}^{2} g_{k i, n}^{2} E\left(u_{k}^{2} \eta_{k, n}^{2} \mid Z_{n}\right) E\left(u_{i}^{2} \eta_{i, n}^{2} \mid Z_{n}\right)+\frac{1}{2} g_{i k, n}^{2} g_{k i, n}^{2}\left(\eta_{k, n}^{4} \mid Z_{n}\right) E\left(u_{i}^{4} \mid Z_{n}\right) \\
& \left.\left.+\frac{1}{2} g_{i k, n}^{2} g_{k i, n}^{2} E\left(\eta_{k, n}^{4} \mid Z_{n}\right) E\left(u_{i}^{2} \eta_{i, n}^{2} \mid Z_{n}\right)\right]\right) \\
\leq & \frac{1}{K_{n}^{2}} \bar{D}_{\xi}^{2} \quad \sum_{1 \leq i<k \leq n}\left[3 E_{Z}\left(g_{i k, n}^{4}\right)+3 E_{Z}\left(g_{k i, n}^{4}\right)+10 E_{Z}\left(g_{i k, n}^{2} g_{k i, n}^{2}\right)\right] \\
= & o(1), \quad(53)
\end{aligned}
$$




$$
\begin{aligned}
\frac{1}{K_{n}^{2}} \sum_{k=1}^{n}\left|\mathcal{E}_{4 k, n}\right| \leq & \frac{4}{K_{n}^{2}} E_{Z}\left(\sum _ { 1 \leq i < j < k \leq n } \left[g_{i k, n}^{2} g_{j k, n}^{2} \sigma_{\eta \eta, i}\left(Z_{n}\right) \sigma_{\eta \eta, j}\left(Z_{n}\right) E\left(u_{k}^{4} \mid Z_{n}\right)\right.\right. \\
& +g_{k i, n}^{2} g_{j k, n}^{2} \sigma_{u u, i}\left(Z_{n}\right) \sigma_{\eta \eta, j}\left(Z_{n}\right) E\left(u_{k}^{2} \eta_{k, n}^{2} \mid Z_{n}\right) \\
& +\frac{1}{2} g_{i k, n}^{2} g_{j k, n}^{2} E\left(u_{k}^{4} \mid Z_{n}\right) \sqrt{\sigma_{u u, i}\left(Z_{n}\right)} \sqrt{\sigma_{\eta \eta, i}\left(Z_{n}\right)} \sigma_{\eta \eta, j}\left(Z_{n}\right) \\
& +\frac{1}{2} g_{k i, n}^{2} g_{j k, n}^{2} E\left(u_{k}^{4} \mid Z_{n}\right) \sqrt{\sigma_{u u, i}\left(Z_{n}\right)} \sqrt{\sigma_{\eta \eta, i}\left(Z_{n}\right)} \sigma_{\eta \eta, j}\left(Z_{n}\right) \\
& +\frac{1}{2} g_{i k, n}^{2} g_{j k, n}^{2} E\left(u_{k}^{2} \eta_{k, n}^{2} \mid Z_{n}\right) \sqrt{\sigma_{u u, i}\left(Z_{n}\right)} \sqrt{\sigma_{\eta \eta, i}\left(Z_{n}\right)} \sigma_{\eta \eta, j}\left(Z_{n}\right) \\
& +\frac{1}{2} g_{k i, n}^{2} g_{j k, n}^{2} E\left(u_{k}^{2} \eta_{k, n}^{2} \mid Z_{n}\right) \sqrt{\sigma_{u u, i}\left(Z_{n}\right)} \sqrt{\sigma_{\eta \eta, i}\left(Z_{n}\right)} \sigma_{\eta \eta, j}\left(Z_{n}\right) \\
& +\frac{1}{2} g_{i k, n}^{2} g_{j k, n}^{2} E\left(u_{k}^{4} \mid Z_{n}\right) \sqrt{\sigma_{u u, j}\left(Z_{n}\right)} \sqrt{\sigma_{\eta \eta, j}\left(Z_{n}\right)} \sigma_{\eta \eta, i}\left(Z_{n}\right) \\
& +\frac{1}{2} g_{i k, n}^{2} g_{k j, n}^{2} E\left(u_{k}^{4} \mid Z_{n}\right) \sqrt{\sigma_{u u, j}\left(Z_{n}\right)} \sqrt{\sigma_{\eta \eta, j}\left(Z_{n}\right)} \sigma_{\eta \eta, i}\left(Z_{n}\right) \\
& +\frac{1}{2} g_{i k, n}^{2} g_{j k, n}^{2} E\left(u_{k}^{2} \eta_{k, n}^{2} \mid Z_{n}\right) \sqrt{\sigma_{u u, j}\left(Z_{n}\right)} \sqrt{\sigma_{\eta \eta, j}\left(Z_{n}\right)} \sigma_{\eta \eta, i}\left(Z_{n}\right) \\
& +\frac{1}{2} g_{i k, n}^{2} g_{k j, n}^{2} E\left(u_{k}^{2} \eta_{k, n}^{2} \mid Z_{n}\right) \sqrt{\sigma_{u u, j}\left(Z_{n}\right)} \sqrt{\sigma_{\eta \eta, j}\left(Z_{n}\right)} \sigma_{\eta \eta, i}\left(Z_{n}\right) \\
& +2 g_{i k, n}^{2} g_{j k, n}^{2} E\left(u_{k}^{2} \eta_{k, n}^{2} \mid Z_{n}\right) \sqrt{\sigma_{u u, i}\left(Z_{n}\right)} \sqrt{\sigma_{\eta \eta, i}\left(Z_{n}\right)} \sqrt{\sigma_{u u, j}\left(Z_{n}\right)} \sqrt{\sigma_{\eta \eta, j}\left(Z_{n}\right)} \\
& \left.\left.+2 g_{k i, n}^{2} g_{k j, n}^{2} E\left(u_{k}^{2} \eta_{k, n}^{2} \mid Z_{n}\right) \sqrt{\sigma_{u u, i}\left(Z_{n}\right)} \sqrt{\sigma_{\eta \eta, i}\left(Z_{n}\right)} \sqrt{\sigma_{u u, j}\left(Z_{n}\right)} \sqrt{\sigma_{\eta \eta, j}\left(Z_{n}\right)}\right]\right) \\
\leq & 4 \bar{D}_{\xi}^{2} \frac{1}{K_{n}^{2}} \sum_{1 \leq i<j<k}\left[5 E_{Z}\left(g_{i k, n}^{2} g_{j k, n}^{2}\right)+2 E_{Z}\left(g_{k i, n}^{2} g_{j k, n}^{2}\right)+2 E_{Z}\left(g_{k i, n}^{2} g_{k j, n}^{2}\right)\right. \\
& \left.+E_{Z}\left(g_{i k, n}^{2} g_{k j, n}^{2}\right)\right] \\
= & o(1), \\
&
\end{aligned}
$$




$$
\begin{aligned}
\frac{1}{K_{n}^{2}} \sum_{k=1}^{n}\left|\mathcal{E}_{5 k, n}\right| \leq & \frac{4}{K_{n}^{2}} E_{Z}\left(\sum _ { 1 \leq i < j < k \leq n } \left[g_{k i, n}^{2} g_{k j, n}^{2} \sigma_{u u, i}\left(Z_{n}\right) \sigma_{u u, j}\left(Z_{n}\right) E\left(\eta_{k, n}^{4} \mid Z_{n}\right)\right.\right. \\
& +g_{i k, n}^{2} g_{k j, n}^{2} E\left(u_{k}^{2} \eta_{k, n}^{2} \mid Z_{n}\right) \sigma_{\eta \eta, i}\left(Z_{n}\right) \sigma_{u u, j}\left(Z_{n}\right) \\
& +\frac{1}{2} g_{k i, n}^{2} g_{j k, n}^{2} E\left(u_{k}^{2} \eta_{k, n}^{2} \mid Z_{n}\right) \sigma_{u u, i}\left(Z_{n}\right) \sqrt{\sigma_{u u, j}\left(Z_{n}\right)} \sqrt{\sigma_{\eta \eta, j}\left(Z_{n}\right)} \\
& +\frac{1}{2} g_{k i, n}^{2} g_{k j, n}^{2} E\left(u_{k}^{2} \eta_{k, n}^{2} \mid Z_{n}\right) \sigma_{u u, i}\left(Z_{n}\right) \sqrt{\sigma_{u u, j}\left(Z_{n}\right)} \sqrt{\sigma_{\eta \eta, j}\left(Z_{n}\right)} \\
& +\frac{1}{2} g_{k i, n}^{2} g_{j k, n}^{2} E\left(\eta_{k, n}^{4} \mid Z_{n}\right) \sigma_{u u, i}\left(Z_{n}\right) \sqrt{\sigma_{u u, j}\left(Z_{n}\right)} \sqrt{\sigma_{\eta \eta, j}\left(Z_{n}\right)} \\
& +\frac{1}{2} g_{k i, n}^{2} g_{k j, n}^{2} E\left(\eta_{k, n}^{4} \mid Z_{n}\right) \sigma_{u u, i}\left(Z_{n}\right) \sqrt{\sigma_{u u, j}\left(Z_{n}\right)} \sqrt{\sigma_{\eta \eta, j}\left(Z_{n}\right)} \\
& +\frac{1}{2} g_{i k, n}^{2} g_{k j, n}^{2} E\left(u_{k}^{2} \eta_{k, n}^{2} \mid Z_{n}\right) \sigma_{u u, j}\left(Z_{n}\right) \sqrt{\sigma_{u u, i}\left(Z_{n}\right)} \sqrt{\sigma_{\eta \eta, i}\left(Z_{n}\right)} \\
& +\frac{1}{2} g_{k i, n}^{2} g_{k j, n}^{2} E\left(u_{k}^{2} \eta_{k, n}^{2} \mid Z_{n}\right) \sigma_{u u, j}\left(Z_{n}\right) \sqrt{\sigma_{u u, i}\left(Z_{n}\right)} \sqrt{\sigma_{\eta \eta, i}\left(Z_{n}\right)} \\
& +\frac{1}{2} g_{i k, n}^{2} g_{k j, n}^{2} E\left(\eta_{k, n}^{4} \mid Z_{n}\right) \sigma_{u u, j}\left(Z_{n}\right) \sqrt{\sigma_{u u, i}\left(Z_{n}\right)} \sqrt{\sigma_{\eta \eta, i}\left(Z_{n}\right)} \\
& \left.\left.+\frac{1}{2} g_{k i, n}^{2} g_{k j, n}^{2} E\left(\eta_{k, n}^{4} \mid Z_{n}\right) \sigma_{u u, j}\left(Z_{n}\right) \sqrt{\sigma_{u u, i}\left(Z_{n}\right)} \sqrt{\sigma_{\eta \eta, i}\left(Z_{n}\right)}\right]\right) \\
\leq & 4 \bar{D}_{\xi}^{2} \sum_{1 \leq i<j<k \leq n}\left[3 E_{Z} g_{k i, n}^{2} g_{k j, n}^{2}+2 E_{Z} g_{i k, n}^{2} g_{k j, n}^{2}+E_{Z} g_{k i, n}^{2} g_{j k, n}^{2}\right] \\
= & \left.o(1),{ }_{1}\right]
\end{aligned}
$$

$$
\begin{aligned}
\frac{1}{K_{n}^{2}} \sum_{k=1}^{n}\left|\mathcal{E}_{6 k, n}\right| \leq & \frac{2}{K_{n}^{2}} E_{Z}\left(\sum _ { 1 \leq i < k \leq n } \left[f_{k, n}^{2} g_{i k, n}^{2} E\left(u_{k}^{4} \mid Z_{n}\right) \sigma_{\eta \eta, i}\left(Z_{n}\right)\right.\right. \\
& +f_{k, n}^{2} g_{k i, n}^{2} E\left(u_{k}^{2} \eta_{k, n}^{2} \mid Z_{n}\right) \sigma_{u u, i}\left(Z_{n}\right) \\
& +\frac{1}{4} f_{k, n}^{2} g_{i k, n}^{2} E\left(\eta_{k, n}^{2} u_{k}^{2} \mid Z_{n}\right) \sqrt{\sigma_{u u, i}\left(Z_{n}\right)} \sqrt{\sigma_{\eta \eta, i}\left(Z_{n}\right)} \\
& +\frac{1}{4} f_{k, n}^{2} g_{k i, n}^{2} E\left(\eta_{k, n}^{2} u_{k}^{2} \mid Z_{n}\right) \sqrt{\sigma_{u u, i}\left(Z_{n}\right)} \sqrt{\sigma_{\eta \eta, i}\left(Z_{n}\right)} \\
& +\frac{1}{4} f_{k, n}^{2} g_{i k, n}^{2} E\left(u_{k}^{4} \mid Z_{n}\right) \sqrt{\sigma_{u u, i}\left(Z_{n}\right)} \sqrt{\sigma_{\eta \eta, i}\left(Z_{n}\right)} \\
& \left.\left.+\frac{1}{4} f_{k, n}^{2} g_{k i, n}^{2} E\left(u_{k}^{4} \mid Z_{n}\right) \sqrt{\sigma_{u u, i}\left(Z_{n}\right)} \sqrt{\sigma_{\eta \eta, i}\left(Z_{n}\right)}\right]\right) \\
\leq & 3 \bar{D}_{\xi}^{\frac{3}{2}} \quad \sum_{1 \leq i<k \leq n}\left[E_{Z}\left(f_{k, n}^{2} g_{i k, n}^{2}\right)+E_{Z}\left(f_{k, n}^{2} g_{k i, n}^{2}\right)\right] \\
= & o(1), \quad{ }^{2},
\end{aligned}
$$




$$
\begin{aligned}
\frac{1}{K_{n}^{2}} \sum_{k=1}^{n}\left|\mathcal{E}_{7 k, n}\right| \leq & \frac{4}{K_{n}^{2}} E_{Z}\left(\sum _ { 1 \leq i < k \leq n } \left[\frac{1}{4} f_{k, n}^{2} g_{i k, n}^{2} E\left(\eta_{i, n}^{4} \mid Z_{n}\right) E\left(u_{k}^{4} \mid Z_{n}\right)\right.\right. \\
& +\frac{1}{4} f_{k, n}^{2} g_{i k, n}^{2} \sigma_{\eta \eta, i}\left(Z_{n}\right) E\left(u_{k}^{4} \mid Z_{n}\right)+\frac{1}{4} g_{i k, n}^{4} E\left(\eta_{i, n}^{4} \mid Z_{n}\right) E\left(u_{k}^{4} \mid Z_{n}\right) \\
& +\frac{1}{4} g_{i k, n}^{4} \sigma_{\eta \eta, i}\left(Z_{n}\right) E\left(u_{k}^{4} \mid Z_{n}\right)+\frac{1}{8} f_{k, n}^{2} g_{k i, n}^{2} E\left(u_{k}^{2} \eta_{k, n}^{2} \mid Z_{n}\right) E\left(u_{i}^{4} \mid Z_{n}\right) \\
& +\frac{1}{8} f_{k, n}^{2} g_{k i, n}^{2} E\left(u_{k}^{2} \eta_{k, n}^{2} \mid Z_{n}\right) \sigma_{u u, i}\left(Z_{n}\right)+\frac{1}{8} f_{k, n}^{2} g_{k i, n}^{2} E\left(\eta_{k, n}^{4} \mid Z_{n}\right) E\left(u_{i}^{4} \mid Z_{n}\right) \\
& +\frac{1}{8} f_{k, n}^{2} g_{k i, n}^{2} E\left(\eta_{k, n}^{4} \mid Z_{n}\right) \sigma_{u u, i}\left(Z_{n}\right)+\frac{1}{8} g_{k i, n}^{4} E\left(u_{k}^{2} \eta_{k, n}^{2} \mid Z_{n}\right) E\left(u_{i}^{4} \mid Z_{n}\right) \\
& +\frac{1}{8} g_{k i, n}^{4} E\left(u_{k}^{2} \eta_{k, n}^{2} \mid Z_{n}\right) \sigma_{u u, i}\left(Z_{n}\right)+\frac{1}{8} g_{k i, n}^{4} E\left(\eta_{k, n}^{4} \mid Z_{n}\right) E\left(u_{i}^{4} \mid Z_{n}\right) \\
& \left.\left.+\frac{1}{8} g_{k i, n}^{4} E\left(\eta_{k, n}^{4} \mid Z_{n}\right) \sigma_{u u, i}\left(Z_{n}\right)\right]\right) \\
\leq & \frac{1}{K_{n}^{2}} \bar{D}_{\xi}^{2} \sum_{1 \leq i<k \leq n}\left[E_{Z}\left(f_{k, n}^{2} g_{i k, n}^{2}\right)+E_{Z}\left(f_{k, n}^{2} g_{k i, n}^{2}\right)+E_{Z}\left(g_{i k, n}^{4}\right)+E_{Z}\left(g_{k i, n}^{4}\right)\right] \\
& +\frac{1}{K_{n}^{2}} \bar{D}_{\xi}^{\frac{3}{2}} \sum_{1 \leq i<k \leq n}\left[E_{Z}\left(f_{k, n}^{2} g_{i k, n}^{2}\right)+E_{Z}\left(f_{k, n}^{2} g_{k i, n}^{2}\right)+E_{Z}\left(g_{i k, n}^{4}\right)+E_{Z}\left(g_{k i, n}^{4}\right)\right] \\
= & o(1), \quad{ }_{i} \quad(57)
\end{aligned}
$$

$$
\begin{aligned}
\frac{1}{K_{n}^{2}} \sum_{k=1}^{n}\left|\mathcal{E}_{8 k, n}\right| \leq & \frac{4}{K_{n}^{2}} E_{Z}\left(\sum _ { 1 \leq i < k \leq n } \left[\frac{3}{4} f_{k, n}^{2} g_{i k, n}^{2} E\left(u_{k}^{2} \eta_{k, n}^{2} \mid Z_{n}\right) E\left(u_{i}^{4} \mid Z_{n}\right)\right.\right. \\
& +\frac{3}{4} f_{k, n}^{2} g_{i k, n}^{2} E\left(u_{k}^{2} \eta_{k, n}^{2} \mid Z_{n}\right) \sigma_{\eta \eta, i}\left(Z_{n}\right)+\frac{3}{4} g_{k i, n}^{4} E\left(u_{k}^{2} \eta_{k, n}^{2} \mid Z_{n}\right) E\left(u_{i}^{4} \mid Z_{n}\right) \\
& +\frac{3}{4} g_{k i, n}^{4} E\left(u_{k}^{2} \eta_{k, n}^{2} \mid Z_{n}\right) \sigma_{\eta \eta, i}\left(Z_{n}\right)+\frac{3}{8} f_{k, n}^{2} g_{k i, n}^{2} E\left(u_{k}^{2} \eta_{k, n}^{2} \mid Z_{n}\right) \sigma_{u u, i}\left(Z_{n}\right) \\
& +\frac{3}{8} f_{k, n}^{2} g_{k i, n}^{2} E\left(u_{k}^{2} \eta_{k, n}^{2} \mid Z_{n}\right) E\left(\eta_{i, n}^{4} \mid Z_{n}\right)+\frac{3}{8} f_{k, n}^{2} g_{k i, n}^{2} E\left(u_{k}^{4} \mid Z_{n}\right) \sigma_{u u, i}\left(Z_{n}\right) \\
& +\frac{3}{8} f_{k, n}^{2} g_{k i, n}^{2} E\left(u_{k}^{4} \mid Z_{n}\right) E\left(\eta_{i, n}^{4} \mid Z_{n}\right)+\frac{3}{8} g_{i k, n}^{4} E\left(u_{k}^{2} \eta_{k, n}^{2} \mid Z_{n}\right) \sigma_{u u, i}\left(Z_{n}\right) \\
& +\frac{3}{8} g_{i k, n}^{4} E\left(u_{k}^{2} \eta_{k, n}^{2} \mid Z_{n}\right) E\left(\eta_{i, n}^{4} \mid Z_{n}\right)+\frac{3}{8} g_{i k, n}^{4} E\left(u_{k}^{4} \mid Z_{n}\right) \sigma_{u u, i}\left(Z_{n}\right) \\
& \left.\left.+\frac{3}{8} g_{i k, n}^{4} E\left(u_{k}^{4} \mid Z_{n}\right) E\left(\eta_{i, n}^{4} \mid Z_{n}\right)\right]\right) \\
\leq & \frac{3}{K_{n}^{2}} \bar{D}_{\xi}^{2} \sum_{1 \leq i<k \leq n}\left[E_{Z}\left(f_{k, n}^{2} g_{i k, n}^{2}\right)+E_{Z}\left(f_{k, n}^{2} g_{k i, n}^{2}\right)+E_{Z}\left(g_{i k, n}^{4}\right)+E_{Z}\left(g_{k i, n}^{4}\right)\right] \\
& +\frac{1}{K_{n}^{2}} \bar{D}_{\xi}^{\frac{3}{2}} \sum_{1 \leq i<k \leq n}\left[E_{Z}\left(f_{k, n}^{2} g_{i k, n}^{2}\right)+E_{Z}\left(f_{k, n}^{2} g_{k i, n}^{2}\right)+E_{Z}\left(g_{i k, n}^{4}\right)+E_{Z}\left(g_{k i, n}^{4}\right)\right] \\
= & \left.o(1), \quad{ }_{1} \sum_{i}\right)
\end{aligned}
$$

where the inequalities in expressions (51)-(58) are obtained by repeated applications of the Cauchy- 
Schwarz and the triangle inequalities and of the inequality $2|X Y| \leq X^{2}+Y^{2}$. From expressions (51)-(58), it follows immediately that

$$
\begin{aligned}
\frac{1}{K_{n}^{2}} \sum_{j=1}^{n} E\left(W_{j n}^{4}\right) & \leq \sum_{i=1}^{8}\left(\frac{1}{K_{n}^{2}} \sum_{j=1}^{n}\left|\mathcal{E}_{i j, n}\right|\right) \\
& =o(1) \text { as } n \rightarrow \infty .
\end{aligned}
$$

\section{Checking Condition C2:}

First define

$$
B_{j n}=\frac{W_{j n}}{\sigma_{W_{n}}},
$$

where $W_{j n}$ is as defined in expression (32). Note that by Lemma A8, $\sigma_{W_{n}}=\sqrt{K_{n}}$. Now, consider the $\sigma$-fields $\mathcal{F}_{j, n}=\sigma\left(\epsilon_{1}, \ldots, \epsilon_{j}, Z_{n}\right), i=1, \ldots, n$, where $\epsilon_{i}=\left(u_{i}, \eta_{i}\right)$ and take $\mathcal{F}_{0, n}$ to be the trivial $\sigma$-field. It follows that by construction that $\mathcal{F}_{j-1, n} \subseteq \mathcal{F}_{j, n}$. Moreover, note that $W_{j n}$ is $\mathcal{F}_{j, n}$-measurable, and straightforward calculation shows that $E\left(W_{j n} \mid \mathcal{F}_{j-1, n}\right)=0$, so that $\left\{W_{j n}, \mathcal{F}_{j, n}, 1 \leq j \leq n, n \geq 1\right\}$ forms a martingale difference array.

Hence, to verify condition 2 , we need to show

$$
\sum_{j=1}^{n} E\left[B_{j, n}^{2} \mid \mathcal{F}_{j-1, n}\right] \stackrel{P}{\rightarrow} 1, \quad \text { as } n \rightarrow \infty
$$

or, alternatively,

$$
\sum_{j=1}^{n}\left(\frac{E\left[W_{j, n}^{2} \mid \mathcal{F}_{j-1, n}\right]-E\left[W_{j, n}^{2}\right]}{\sigma_{W_{n}}^{2}}\right) \stackrel{p}{\rightarrow} 0, \quad \text { as } n \rightarrow \infty
$$

or, equivalently,

$$
\frac{1}{K_{n}^{2}} E\left(\sum_{j=1}^{n}\left\{E\left[W_{j, n}^{2} \mid \mathcal{F}_{j-1, n}\right]-E\left[W_{j, n}^{2}\right]\right\}\right)^{2} \rightarrow 0, \quad \text { as } n \rightarrow \infty,
$$

in light of Lemma A8. To show (62), we proceed by noting that 


$$
\begin{aligned}
& \sum_{k=1}^{n}\left(E\left[W_{k, n}^{2} \mid \mathcal{F}_{k-1, n}\right]-E\left[W_{k, n}^{2}\right]\right) \\
= & 2 \sum_{1 \leq i<k \leq n}\left[f_{k, n} g_{i k, n} \eta_{i, n} E\left(u_{k}^{2} \mid \mathcal{F}_{k-1, n}\right)+f_{k, n} g_{k i, n} u_{i} E\left(\eta_{k, n} u_{k} \mid \mathcal{F}_{k-1, n}\right)\right] \\
& +\sum_{1 \leq i<k \leq n}\left[g_{i k, n}^{2} \eta_{i, n}^{2} E\left(u_{k}^{2} \mid \mathcal{F}_{k-1, n}\right)-E_{Z}\left(g_{i k, n}^{2} \sigma_{\eta \eta, i}\left(Z_{n}\right) \sigma_{u u, k}\left(Z_{n}\right)\right)\right] \\
& +\sum_{1 \leq i<k \leq n}\left[g_{k i, n}^{2} u_{i}^{2} E\left(\eta_{k, n}^{2} \mid \mathcal{F}_{k-1, n}\right)-E_{Z}\left(g_{k i, n}^{2} \sigma_{u u, i}\left(Z_{n}\right) \sigma_{\eta \eta, k}\left(Z_{n}\right)\right)\right] \\
& +2 \sum_{1 \leq i<k \leq n}\left[g_{i k, n} g_{k i, n} \eta_{i, n} u_{i} E\left(\eta_{k, n} u_{k} \mid \mathcal{F}_{k-1, n}\right)-E_{Z}\left(g_{i k, n} g_{k i, n} \sigma_{\eta u, i}\left(Z_{n}\right) \sigma_{\eta u, k}\left(Z_{n}\right)\right)\right] \\
& +2 \sum_{1 \leq i<j<k \leq n}\left[g_{i k, n} g_{j k, n} \eta_{i, n} \eta_{j, n} E\left(u_{k}^{2} \mid \mathcal{F}_{k-1, n}\right)+g_{k i, n} g_{j k, n} u_{i} \eta_{j, n} E\left(\eta_{k, n} u_{k} \mid \mathcal{F}_{k-1, n}\right)\right. \\
& \left.+g_{i k, n} g_{k j, n} \eta_{i, n} u_{j} E\left(\eta_{k, n} u_{k} \mid \mathcal{F}_{k-1, n}\right)+g_{k i, n} g_{k j, n} u_{i} u_{j} E\left(\eta_{k, n}^{2} \mid \mathcal{F}_{k-1, n}\right)\right]
\end{aligned}
$$

Further calculations yield

$$
\frac{1}{K_{n}^{2}} E\left(\sum_{j=1}^{n}\left\{E\left[W_{j, n}^{2} \mid \mathcal{F}_{j-1, n}\right]-E\left[W_{j, n}^{2}\right]\right\}\right)^{2}=\frac{1}{K_{n}^{2}} \sum_{i=1}^{7} \mathcal{A}_{i, n}
$$

where

$$
\begin{aligned}
\mathcal{A}_{1, n}= & 4 E_{Z}\left(\sum _ { 1 \leq i < k \leq n } \left[f_{k, n}^{2} g_{i k, n}^{2} \sigma_{\eta \eta, i}\left(Z_{n}\right) \sigma_{u u, k}^{2}\left(Z_{n}\right)+f_{k, n}^{2} g_{k i, n}^{2} \sigma_{u u, i}\left(Z_{n}\right) \sigma_{\eta u, k}^{2}\left(Z_{n}\right)\right.\right. \\
& \left.+2 f_{k, n}^{2} g_{i k, n} g_{k i, n} \sigma_{\eta u, i}\left(Z_{n}\right) \sigma_{u u, k}\left(Z_{n}\right) \sigma_{\eta u, k}\left(Z_{n}\right)\right] \\
& +2 \sum_{1 \leq i<j<k \leq n}\left[f_{j, n} f_{k, n} g_{i j, n} g_{i k, n} \sigma_{\eta \eta, i}\left(Z_{n}\right) \sigma_{u u, j}\left(Z_{n}\right) \sigma_{u u, k}\left(Z_{n}\right)\right. \\
& +f_{j, n} f_{k, n} g_{j i, n} g_{k i, n} \sigma_{u u, i}\left(Z_{n}\right) \sigma_{\eta u, j}\left(Z_{n}\right) \sigma_{\eta u, k}\left(Z_{n}\right) \\
& +f_{j, n} f_{k, n} g_{i j, n} g_{k i, n} \sigma_{\eta u, i}\left(Z_{n}\right) \sigma_{u u, j}\left(Z_{n}\right) \sigma_{\eta u, k}\left(Z_{n}\right) \\
& \left.\left.+f_{j, n} f_{k, n} g_{j i, n} g_{i k, n} \sigma_{\eta u, i}\left(Z_{n}\right) \sigma_{\eta u, j}\left(Z_{n}\right) \sigma_{u u, k}\left(Z_{n}\right)\right]\right)
\end{aligned}
$$




$$
\begin{aligned}
\mathcal{A}_{2, n}= & \sum_{1 \leq i<k \leq n}\left(\left\{E_{Z}\left[g_{i k, n}^{4} \sigma_{u u, k}^{2}\left(Z_{n}\right) E\left(\eta_{i, n}^{4} \mid Z_{n}\right)\right]-\left(E_{Z}\left[g_{i k, n}^{2} \sigma_{u u, k}\left(Z_{n}\right) \sigma_{\eta \eta, i}\left(Z_{n}\right)\right]\right)^{2}\right\}\right. \\
& +\left\{E_{Z}\left[g_{k i, n}^{4} \sigma_{\eta \eta, k}^{2}\left(Z_{n}\right) E\left(u_{i}^{4} \mid Z_{n}\right)\right]-\left(E_{Z}\left[g_{k i, n}^{2} \sigma_{\eta \eta, k}\left(Z_{n}\right) \sigma_{u u, i}\left(Z_{n}\right)\right]\right)^{2}\right\} \\
& +4\left\{E_{Z}\left[g_{i k, n}^{2} g_{k i, n}^{2} \sigma_{\eta u, k}^{2}\left(Z_{n}\right) E\left(\eta_{i, n}^{2} u_{i}^{2} \mid Z_{n}\right)\right]-\left(E_{Z}\left[g_{i k, n} g_{k i, n} \sigma_{\eta u, k}\left(Z_{n}\right) \sigma_{\eta u, i}\left(Z_{n}\right)\right]\right)^{2}\right\} \\
& +2\left\{E_{Z}\left[g_{i k, n}^{2} g_{k i, n}^{2} \sigma_{u u, k}\left(Z_{n}\right) \sigma_{\eta \eta, k}\left(Z_{n}\right) E\left(\eta_{i, n}^{2} u_{i}^{2} \mid Z_{n}\right)\right]\right. \\
& \left.-E_{Z}\left[g_{i k, n}^{2} \sigma_{u u, k}\left(Z_{n}\right) \sigma_{\eta \eta, i}\left(Z_{n}\right)\right] E_{Z}\left[g_{k i, n}^{2} \sigma_{u u, i}\left(Z_{n}\right) \sigma_{\eta \eta, k}\left(Z_{n}\right)\right]\right\} \\
& +4\left\{E_{Z}\left[g_{i k, n}^{3} g_{k i, n} \sigma_{u u, k}\left(Z_{n}\right) \sigma_{\eta u, k}\left(Z_{n}\right) E\left(\eta_{i, n}^{3} u_{i} \mid Z_{n}\right)\right]\right. \\
& \left.-E_{Z}\left[g_{i k, n}^{2} \sigma_{\eta \eta, i}\left(Z_{n}\right) \sigma_{u u, k}\left(Z_{n}\right)\right] E_{Z}\left[g_{i k, n} g_{k i, n} \sigma_{\eta u, i}\left(Z_{n}\right) \sigma_{\eta u, k}\left(Z_{n}\right)\right]\right\} \\
& +4\left\{E_{Z}\left[g_{i k, n} g_{k i, n}^{3} \sigma_{\eta \eta, k}\left(Z_{n}\right) \sigma_{\eta u, k}\left(Z_{n}\right) E\left(\eta_{i, n} u_{i}^{3} \mid Z_{n}\right)\right]\right. \\
& \left.\left.-E_{Z}\left[g_{k i, n}^{2} \sigma_{u u, i}\left(Z_{n}\right) \sigma_{\eta \eta, k}\left(Z_{n}\right)\right] E_{Z}\left[g_{i k, n} g_{k i, n} \sigma_{\eta u, i}\left(Z_{n}\right) \sigma_{\eta u, k}\left(Z_{n}\right)\right]\right\}\right)
\end{aligned}
$$

$$
\begin{aligned}
\mathcal{A}_{3, n}= & \sum_{1 \leq i<j<k \leq n}\left(\left\{E_{Z}\left[g_{i j, n}^{2} g_{i k, n}^{2} E\left(\eta_{i, n}^{4} \mid Z_{n}\right) \sigma_{u u, j}\left(Z_{n}\right) \sigma_{u u, k}\left(Z_{n}\right)\right]\right.\right. \\
& \left.-E_{Z}\left[g_{i j, n}^{2} \sigma_{u u, j}\left(Z_{n}\right) \sigma_{\eta \eta, i}\left(Z_{n}\right)\right] E_{Z}\left[g_{i k, n}^{2} \sigma_{u u, k}\left(Z_{n}\right) \sigma_{\eta \eta, i}\left(Z_{n}\right)\right]\right\} \\
& +\left\{E_{Z}\left[g_{j i, n}^{2} g_{k i, n}^{2} E\left(u_{i}^{4} \mid Z_{n}\right) \sigma_{\eta \eta, j}^{2}\left(Z_{n}\right) \sigma_{\eta \eta, k}^{2}\left(Z_{n}\right)\right]\right. \\
& \left.-E_{Z}\left[g_{j i, n}^{2} \sigma_{\eta \eta, j}\left(Z_{n}\right) \sigma_{u u, i}\left(Z_{n}\right)\right] E_{Z}\left[g_{k i, n}^{2} \sigma_{\eta \eta, k}\left(Z_{n}\right) \sigma_{u u, i}\left(Z_{n}\right)\right]\right\} \\
& +4\left\{E_{Z}\left[g_{i j, n} g_{j i, n} g_{i k, n} g_{k i, n} \sigma_{\eta u, j}\left(Z_{n}\right) \sigma_{\eta u, k}\left(Z_{n}\right) E\left(\eta_{i, n}^{2} u_{i}^{2} \mid Z_{n}\right)\right]\right. \\
& \left.\left.-E_{Z}\left[g_{i j, n} g_{j i, n} \sigma_{\eta u, i}\left(Z_{n}\right) \sigma_{\eta u, j}\left(Z_{n}\right)\right] E_{Z}\left[g_{i k, n} g_{k i, n} \sigma_{\eta u, i}\left(Z_{n}\right) \sigma_{\eta u, k}\left(Z_{n}\right)\right]\right\}\right)
\end{aligned}
$$




$$
\begin{aligned}
\mathcal{A}_{4, n}= & \sum_{1 \leq i<j<k \leq n}\left(\left\{E_{Z}\left[g_{i j, n}^{2} g_{k i, n}^{2} \sigma_{u u, j}\left(Z_{n}\right) \sigma_{\eta \eta, k}\left(Z_{n}\right) E\left(\eta_{i, n}^{2} u_{i}^{2} \mid Z_{n}\right)\right]\right.\right. \\
& \left.-E_{Z}\left[g_{i j, n}^{2} \sigma_{u u, j}\left(Z_{n}\right) \sigma_{\eta \eta, i}\left(Z_{n}\right)\right] E_{Z}\left[g_{k i, n}^{2} \sigma_{u u, i}\left(Z_{n}\right) \sigma_{\eta \eta, k}\left(Z_{n}\right)\right]\right\} \\
& +\left\{E_{Z}\left[g_{i k, n}^{2} g_{j i, n}^{2} \sigma_{u u, k}\left(Z_{n}\right) \sigma_{\eta \eta, j}\left(Z_{n}\right) E\left(\eta_{i, n}^{2} u_{i}^{2} \mid Z_{n}\right)\right]\right. \\
& \left.-E_{Z}\left[g_{i k, n}^{2} \sigma_{u u, k}\left(Z_{n}\right) \sigma_{\eta \eta, i}\left(Z_{n}\right)\right] E_{Z}\left[g_{j i, n}^{2} \sigma_{u u, i}\left(Z_{n}\right) \sigma_{\eta \eta, j}\left(Z_{n}\right)\right]\right\} \\
& +2\left\{E_{Z}\left[g_{i j, n}^{2} g_{i k, n} g_{k i, n} \sigma_{u u, j}\left(Z_{n}\right) \sigma_{\eta u, k}\left(Z_{n}\right) E\left(\eta_{i, n}^{3} u_{i} \mid Z_{n}\right)\right]\right. \\
& \left.-E_{Z}\left[g_{i j, n}^{2} \sigma_{\eta \eta, i}\left(Z_{n}\right) \sigma_{u u, j}\left(Z_{n}\right)\right] E_{Z}\left[g_{i k, n} g_{k i, n} \sigma_{\eta u, i}\left(Z_{n}\right) \sigma_{\eta u, k}\left(Z_{n}\right)\right]\right\} \\
& +2\left\{E_{Z}\left[g_{i k, n}^{2} g_{i j, n} g_{j i, n} \sigma_{u u, k}\left(Z_{n}\right) \sigma_{\eta u, j}\left(Z_{n}\right) E\left(\eta_{i, n}^{3} u_{i} \mid Z_{n}\right)\right]\right. \\
& \left.-E_{Z}\left[g_{i k, n}^{2} \sigma_{\eta \eta, i}\left(Z_{n}\right) \sigma_{u u, k}\left(Z_{n}\right)\right] E_{Z}\left[g_{i j, n} g_{j i, n} \sigma_{\eta u, i}\left(Z_{n}\right) \sigma_{\eta u, j}\left(Z_{n}\right)\right]\right\} \\
& +2\left\{E_{Z}\left[g_{k i, n}^{2} g_{i j, n} g_{j i, n} \sigma_{\eta \eta, k}\left(Z_{n}\right) \sigma_{\eta u, j}\left(Z_{n}\right) E\left(\eta_{i, n} u_{i}^{3} \mid Z_{n}\right)\right]\right. \\
& -E_{Z}\left[g_{j i, n}^{2} \sigma_{u u, i}\left(Z_{n}\right) \sigma_{\eta \eta, j}\left(Z_{n}\right)\right] E_{Z}\left[g_{i k, n} g_{k i, n} \sigma_{\eta u, i}\left(Z_{n}\right) \sigma_{\eta u, k}\left(Z_{n}\right)\right] \\
& +2\left\{E_{Z}\left[g_{j i, n}^{2} g_{i k, n} g_{k i, n} \sigma_{\eta \eta, j}\left(Z_{n}\right) \sigma_{\eta u, k}\left(Z_{n}\right) E\left(\eta_{i, n} u_{i}^{3} \mid Z_{n}\right)\right]\right. \\
& \left.\left.-E_{Z}\left[g_{k i, n}^{2} \sigma_{u u, i}\left(Z_{n}\right) \sigma_{\eta \eta, k}\left(Z_{n}\right)\right] E_{Z}\left[g_{i j, n} g_{j i, n} \sigma_{\eta u, i}\left(Z_{n}\right) \sigma_{\eta u, j}\left(Z_{n}\right)\right]\right\}\right)
\end{aligned}
$$

$$
\begin{aligned}
\mathcal{A}_{5, n}= & 4 E_{Z}\left[\sum_{1 \leq i<j<k \leq n} g_{i k, n}^{2} g_{j k, n}^{2} \sigma_{\eta \eta, i}\left(Z_{n}\right) \sigma_{\eta \eta, j}\left(Z_{n}\right) \sigma_{u u, k}^{2}\left(Z_{n}\right)\right. \\
& +g_{k i, n}^{2} g_{j k, n}^{2} \sigma_{u u, i}\left(Z_{n}\right) \sigma_{\eta \eta, j}\left(Z_{n}\right) \sigma_{\eta u, k}^{2}\left(Z_{n}\right)+g_{i k, n}^{2} g_{j k, n}^{2} \sigma_{\eta \eta, i}\left(Z_{n}\right) \sigma_{u u, j}\left(Z_{n}\right) \sigma_{\eta u, k}^{2}\left(Z_{n}\right) \\
& +g_{k i, n}^{2} g_{k j, n}^{2} \sigma_{u u, i}\left(Z_{n}\right) \sigma_{u u, j}\left(Z_{n}\right) \sigma_{\eta \eta, k}^{2}\left(Z_{n}\right) \\
& +2 g_{i k, n} g_{k i, n} g_{j k, n}^{2} \sigma_{\eta u, i}\left(Z_{n}\right) \sigma_{\eta \eta, j}\left(Z_{n}\right) \sigma_{u u, k}\left(Z_{n}\right) \sigma_{\eta u, k}\left(Z_{n}\right) \\
& +2 g_{i k, n}^{2} g_{j k, n} g_{k j, n} \sigma_{\eta \eta, i}\left(Z_{n}\right) \sigma_{u \eta, j}\left(Z_{n}\right) \sigma_{u u, k}\left(Z_{n}\right) \sigma_{\eta u, k}\left(Z_{n}\right) \\
& +2 g_{i k, n} g_{k i, n} g_{j k, n} g_{k j, n} \sigma_{\eta u, i}\left(Z_{n}\right) \sigma_{\eta u, j}\left(Z_{n}\right) \sigma_{u u, k}\left(Z_{n}\right) \sigma_{\eta \eta, k}\left(Z_{n}\right) \\
& +2 g_{i k, n} g_{k i, n} g_{j k, n} g_{k j, n} \sigma_{\eta u, i}\left(Z_{n}\right) \sigma_{\eta u, j}\left(Z_{n}\right) \sigma_{\eta u, k}^{2}\left(Z_{n}\right) \\
& +2 g_{k i, n}^{2} g_{j k, n} g_{k j, n} \sigma_{u u, i}\left(Z_{n}\right) \sigma_{\eta u, j}\left(Z_{n}\right) \sigma_{\eta u, k}\left(Z_{n}\right) \sigma_{\eta \eta, k}\left(Z_{n}\right) \\
& \left.+2 g_{i k, n} g_{k i, n} g_{k j, n}^{2} \sigma_{\eta u, i}\left(Z_{n}\right) \sigma_{u u, j}\left(Z_{n}\right) \sigma_{\eta u, k}\left(Z_{n}\right) \sigma_{\eta \eta, k}\left(Z_{n}\right)\right]
\end{aligned}
$$




$$
\begin{aligned}
\mathcal{A}_{6, n}= & 2 E_{Z}\left[\sum_{1 \leq i<j<k<l \leq n} g_{i k, n} g_{j k, n} g_{i l, n} g_{j l, n} \sigma_{\eta \eta, i}\left(Z_{n}\right) \sigma_{\eta \eta, j}\left(Z_{n}\right) \sigma_{u u, k}\left(Z_{n}\right) \sigma_{u u, l}\left(Z_{n}\right)\right. \\
& +g_{k i, n} g_{j k, n} g_{l i, n} g_{j l, n} \sigma_{u u, i}\left(Z_{n}\right) \sigma_{\eta \eta, j}\left(Z_{n}\right) \sigma_{\eta u, k}\left(Z_{n}\right) \sigma_{\eta u, l}\left(Z_{n}\right) \\
& +g_{i k, n} g_{k j, n} g_{i l, n} g_{l j, n} \sigma_{\eta \eta, i}\left(Z_{n}\right) \sigma_{u u, j}\left(Z_{n}\right) \sigma_{\eta u, k}\left(Z_{n}\right) \sigma_{\eta u, l}\left(Z_{n}\right) \\
& +g_{k i, n} g_{k j, n} g_{l i, n} g_{l j, n} \sigma_{u u, i}\left(Z_{n}\right) \sigma_{u u, j}\left(Z_{n}\right) \sigma_{\eta \eta, k}\left(Z_{n}\right) \sigma_{\eta \eta, l}\left(Z_{n}\right) \\
& +g_{i k, n} g_{j k, n} g_{l i, n} g_{j l, n} \sigma_{\eta u, i}\left(Z_{n}\right) \sigma_{\eta \eta, j}\left(Z_{n}\right) \sigma_{u u, k}\left(Z_{n}\right) \sigma_{\eta u, l}\left(Z_{n}\right) \\
& +g_{i l, n} g_{j l, n} g_{k i, n} g_{j k, n} \sigma_{\eta u, i}\left(Z_{n}\right) \sigma_{\eta \eta, j}\left(Z_{n}\right) \sigma_{u u, l}\left(Z_{n}\right) \sigma_{\eta u, k}\left(Z_{n}\right) \\
& +g_{i k, n} g_{j k, n} g_{i l, n} g_{l j, n} \sigma_{\eta \eta, i}\left(Z_{n}\right) \sigma_{\eta u, j}\left(Z_{n}\right) \sigma_{u u, k}\left(Z_{n}\right) \sigma_{\eta u, l}\left(Z_{n}\right) \\
& +g_{i l, n} g_{j l, n} g_{i k, n} g_{k j, n} \sigma_{\eta \eta, i}\left(Z_{n}\right) \sigma_{\eta u, j}\left(Z_{n}\right) \sigma_{\eta u, k}\left(Z_{n}\right) \sigma_{u u, l}\left(Z_{n}\right) \\
& +g_{i k, n} g_{j k, n} g_{l i, n} g_{l j, n} \sigma_{\eta u, i}\left(Z_{n}\right) \sigma_{\eta u, j}\left(Z_{n}\right) \sigma_{u u, k}\left(Z_{n}\right) \sigma_{\eta \eta, l}\left(Z_{n}\right) \\
& +g_{i l, n} g_{j l, n} g_{k i, n} g_{k j, n} \sigma_{\eta u, i}\left(Z_{n}\right) \sigma_{\eta u, j}\left(Z_{n}\right) \sigma_{u u, l}\left(Z_{n}\right) \sigma_{\eta \eta, k}\left(Z_{n}\right) \\
& +g_{k i, n} g_{j k, n} g_{i l, n} g_{l j, n} \sigma_{\eta u, i}\left(Z_{n}\right) \sigma_{\eta u, j}\left(Z_{n}\right) \sigma_{\eta u, k}\left(Z_{n}\right) \sigma_{\eta u, l}\left(Z_{n}\right) \\
& +g_{l i, n} g_{j l, n} g_{i k, n} g_{k j, n} \sigma_{\eta u, i}\left(Z_{n}\right) \sigma_{\eta u, j}\left(Z_{n}\right) \sigma_{\eta u, k}\left(Z_{n}\right) \sigma_{\eta u, l}\left(Z_{n}\right) \\
& +g_{k i, n} g_{j k, n} g_{l i, n} g_{l j, n} \sigma_{u u, i}\left(Z_{n}\right) \sigma_{\eta u, j}\left(Z_{n}\right) \sigma_{\eta u, k}\left(Z_{n}\right) \sigma_{\eta \eta, l}\left(Z_{n}\right) \\
& +g_{l i, n} g_{j l, n} g_{k i, n} g_{k j, n} \sigma_{u u, i}\left(Z_{n}\right) \sigma_{\eta u, j}\left(Z_{n}\right) \sigma_{\eta u, l}\left(Z_{n}\right) \sigma_{\eta \eta, k}\left(Z_{n}\right) \\
& +g_{i k, n} g_{k j, n} g_{l i, n} g_{l j, n} \sigma_{\eta u, i}\left(Z_{n}\right) \sigma_{u u, j}\left(Z_{n}\right) \sigma_{\eta u, k}\left(Z_{n}\right) \sigma_{\eta \eta, l}\left(Z_{n}\right) \\
& \left.+g_{i l, n} g_{l j, n} g_{k i, n} g_{k j, n} \sigma_{\eta u, i}\left(Z_{n}\right) \sigma_{u u, j}\left(Z_{n}\right) \sigma_{\eta u, l}\left(Z_{n}\right) \sigma_{\eta \eta, k}\left(Z_{n}\right)\right]
\end{aligned}
$$

Again, making use of Lemmas A5, A6, and A7 and Assumptions 1-4 and 6, we see that

$$
\begin{aligned}
\frac{1}{K_{n}^{2}}\left|\mathcal{A}_{1, n}\right| \leq & \frac{12}{K_{n}^{2}} \bar{D}_{\xi}^{\frac{3}{2}} \sum_{1 \leq i<k \leq n}\left[E_{Z}\left(f_{k, n}^{2} g_{i k, n}^{2}\right)+E_{Z}\left(f_{k, n}^{2} g_{k i, n}^{2}\right)\right] \\
& +8 \mid \frac{1}{K_{n}^{2}} \sum_{1 \leq i<j<k \leq n}\left[E_{Z}\left(f_{j, n} f_{k, n} g_{i j, n} g_{i k, n} \sigma_{\eta \eta, i}\left(Z_{n}\right) \sigma_{u u, j}\left(Z_{n}\right) \sigma_{u u, k}\left(Z_{n}\right)\right)\right. \\
& +E_{Z}\left(f_{j, n} f_{k, n} g_{j i, n} g_{k i, n} \sigma_{u u, i}\left(Z_{n}\right) \sigma_{\eta u, j}\left(Z_{n}\right) \sigma_{\eta u, k}\left(Z_{n}\right)\right) \\
& +E_{Z}\left(f_{j, n} f_{k, n} g_{i j, n} g_{k i, n} \sigma_{\eta u, i}\left(Z_{n}\right) \sigma_{u u, j}\left(Z_{n}\right) \sigma_{\eta u, k}\left(Z_{n}\right)\right) \\
& \left.+E_{Z}\left(f_{j, n} f_{k, n} g_{j i, n} g_{i k, n} \sigma_{\eta u, i}\left(Z_{n}\right) \sigma_{\eta u, j}\left(Z_{n}\right) \sigma_{u u, k}\left(Z_{n}\right)\right)\right] \mid \\
= & o(1)
\end{aligned}
$$




$$
\begin{aligned}
& \frac{1}{K_{n}^{2}}\left|\mathcal{A}_{2, n}\right| \leq \frac{1}{K_{n}^{2}} \bar{D}_{\xi}^{2} \sum_{1 \leq i<k \leq n}\left[4 E_{Z}\left(g_{i k, n}^{4}+g_{k i, n}^{4}\right)+14 E_{Z}\left(g_{i k, n}^{2} g_{k i, n}^{2}\right)\right] \\
& +\frac{1}{K_{n}^{2}} \bar{D}_{\xi}^{2}\left(\frac{1}{1-\bar{h}}\right)^{2} \sum_{1 \leq i<k \leq n}\left[6 E_{Z}\left(g_{i k, n}^{4}\right)+4 E_{Z}\left(g_{k i, n}^{4}\right)\right] \\
& =o(1) \text {, } \\
& \frac{1}{K_{n}^{2}}\left|\mathcal{A}_{3, n}\right| \leq \frac{3}{K_{n}^{2}} \bar{D}_{\xi}^{2} \sum_{1 \leq i<j<k \leq n} E_{Z}\left(g_{i j, n}^{2} g_{i k, n}^{2}+g_{j i, n}^{2} g_{k i, n}^{2}\right) \\
& +\frac{3}{K_{n}^{2}} \bar{D}_{\xi}^{2} \sum_{1 \leq i<j<k \leq n}\left[E_{Z}\left(g_{i j, n}^{2}\right) E_{Z}\left(g_{i k, n}^{2}\right)+E_{Z}\left(g_{j i, n}^{2}\right) E_{Z}\left(g_{k i, n}^{2}\right)\right] \\
& =o(1) \\
& \frac{1}{K_{n}^{2}}\left|\mathcal{A}_{4, n}\right| \leq \frac{3}{K_{n}^{2}} \bar{D}_{\xi}^{2} \sum_{1 \leq i<j<k \leq n} E_{Z}\left(g_{i j, n}^{2} g_{k i, n}^{2}+g_{i k, n}^{2} g_{j i, n}^{2}\right) \\
& +\frac{2}{K_{n}^{2}} \bar{D}_{\xi}^{2} \sum_{1 \leq i<j<k \leq n} E_{Z}\left(g_{i j, n}^{2} g_{i k, n}^{2}+g_{k i, n}^{2} g_{j i, n}^{2}\right) \\
& +\frac{3}{K_{n}^{2}} \bar{D}_{\xi}^{2} \sum_{1 \leq i<j<k \leq n}\left[E_{Z}\left(g_{i j, n}^{2}\right) E_{Z}\left(g_{k i, n}^{2}\right)+E_{Z}\left(g_{i k, n}^{2}\right) E_{Z}\left(g_{j i, n}^{2}\right)\right] \\
& +\frac{2}{K_{n}^{2}} \bar{D}_{\xi}^{2} \sum_{1 \leq i<j<k \leq n}\left[E_{Z}\left(g_{i j, n}^{2}\right) E_{Z}\left(g_{i k, n}^{2}\right)+E_{Z}\left(g_{k i, n}^{2}\right) E_{Z}\left(g_{j i, n}^{2}\right)\right] \\
& =o(1) \\
& \frac{1}{K_{n}^{2}}\left|\mathcal{A}_{5, n}\right| \leq \frac{4}{K_{n}^{2}} \bar{D}_{\xi}^{2} \sum_{1 \leq i<j<k \leq n}\left[6 E_{Z}\left(g_{i k, n}^{2} g_{j k, n}^{2}\right)+4 E_{Z}\left(g_{k i, n}^{2} g_{k j, n}^{2}\right)\right. \\
& \left.+3 E_{Z}\left(g_{k i, n}^{2} g_{j k, n}^{2}\right)+2 E_{Z}\left(g_{i k, n}^{2} g_{k j, n}^{2}\right)\right] \\
& =o(1)
\end{aligned}
$$

where the inequalities in expressions (65)-(69) have been obtained by repeated applications of the Cauchy-Schwarz and the triangle inequalities and of the inequality $2|X Y| \leq X^{2}+Y^{2}$.

Finally, to show that $K_{n}^{-2}\left|\mathcal{A}_{6, n}\right|=o(1)$, let

$$
\begin{aligned}
& \Delta_{4 n}=\sum_{1 \leq i<j<k \leq n}\left[g_{i k, n} g_{j k, n} \eta_{i, n} \eta_{j, n} \sigma_{u u, k}\left(Z_{n}\right)+g_{k i, n} g_{j k, n} u_{i} \eta_{j, n} \sigma_{u \eta, k}\left(Z_{n}\right)\right. \\
& \left.+g_{i k, n} g_{k j, n} \eta_{i, n} u_{j} \sigma_{\eta u, k}\left(Z_{n}\right)+g_{k i, n} g_{k j, n} u_{i} u_{j} \sigma_{\eta \eta, k}\left(Z_{n}\right)\right]
\end{aligned}
$$


and note that $E_{Z}\left(\Delta_{4 n}^{2}\right)=\mathcal{A}_{5, n}+\mathcal{A}_{6, n}$, where $\mathcal{A}_{5, n}$ and $\mathcal{A}_{6, n}$ are as defined in expressions (63) and (64) above. Since we have already shown that $K_{n}^{-2}\left|\mathcal{A}_{5, n}\right|=o(1)$ in expression (69) above, it follows that showing $K_{n}^{-2}\left|\mathcal{A}_{6, n}\right|=o(1)$ is equivalent to showing that $K_{n}^{-2} E_{Z}\left(\Delta_{4 n}^{2}\right)=o(1)$. To show the latter, we define

$$
\begin{aligned}
\Delta_{5 n}= & \sum_{1 \leq i<j<k \leq n}\left[g_{i k, n} g_{j k, n} \eta_{i, n} \eta_{j, n} \sigma_{u u, k}\left(Z_{n}\right)+g_{k i, n} g_{j k, n} u_{i} \eta_{j, n} \sigma_{u \eta, k}\left(Z_{n}\right)\right. \\
& +g_{i k, n} g_{k j, n} \eta_{i, n} u_{j} \sigma_{\eta u, k}\left(Z_{n}\right)+g_{k i, n} g_{k j, n} u_{i} u_{j} \sigma_{\eta \eta, k}\left(Z_{n}\right) \\
& +g_{i j, n} g_{k j, n} \eta_{i, n} \eta_{k, n} \sigma_{u u, j}\left(Z_{n}\right)+g_{j i, n} g_{k j, n} u_{i} \eta_{k, n} \sigma_{\eta u, j}\left(Z_{n}\right) \\
& +g_{i j, n} g_{j k, n} \eta_{i, n} u_{k} \sigma_{\eta u, j}\left(Z_{n}\right)+g_{j i, n} g_{j k, n} u_{i} u_{k} \sigma_{\eta \eta, j}\left(Z_{n}\right) \\
& +g_{j i, n} g_{k i, n} \eta_{j, n} \eta_{k, n} \sigma_{u u, i}\left(Z_{n}\right)+g_{i j, n} g_{k i, n} u_{j} \eta_{k, n} \sigma_{\eta u, i}\left(Z_{n}\right) \\
& \left.+g_{j i, n} g_{i k, n} \eta_{j, n} u_{k} \sigma_{\eta u, i}\left(Z_{n}\right)+g_{i j, n} g_{i k, n} u_{j} u_{k} \sigma_{\eta \eta, i}\left(Z_{n}\right)\right]
\end{aligned}
$$

and

$$
\begin{aligned}
\Delta_{6 n}= & \sum_{1 \leq i<j<k \leq n}\left[g_{i j, n} g_{k j, n} \eta_{i, n} \eta_{k, n} \sigma_{u u, j}\left(Z_{n}\right)+g_{j i, n} g_{k j, n} u_{i} \eta_{k, n} \sigma_{\eta u, j}\left(Z_{n}\right)\right. \\
& +g_{i j, n} g_{j k, n} \eta_{i, n} u_{k} \sigma_{\eta u, j}\left(Z_{n}\right)+g_{j i, n} g_{j k, n} u_{i} u_{k} \sigma_{\eta \eta, j}\left(Z_{n}\right) \\
& +g_{j i, n} g_{k i, n} \eta_{j, n} \eta_{k, n} \sigma_{u u, i}\left(Z_{n}\right)+g_{i j, n} g_{k i, n} u_{j} \eta_{k, n} \sigma_{\eta u, i}\left(Z_{n}\right) \\
& \left.+g_{j i, n} g_{i k, n} \eta_{j, n} u_{k} \sigma_{\eta u, i}\left(Z_{n}\right)+g_{i j, n} g_{i k, n} u_{j} u_{k} \sigma_{\eta \eta, i}\left(Z_{n}\right)\right]
\end{aligned}
$$

and note that $\Delta_{4 n}=\Delta_{5 n}-\Delta_{6 n}$, so that

$$
E\left(\Delta_{4 n}^{2}\right)=E\left(\Delta_{5 n}^{2}\right)+E\left(\Delta_{6 n}^{2}\right)-2 E\left(\Delta_{5 n} \Delta_{6 n}\right) .
$$

By direct calculation, we obtain

$$
\begin{aligned}
& E\left(\Delta_{5 n}^{2}\right)=\left(E_{Z}\left(\mathcal{T}_{5}\right)+E_{Z}\left(\mathcal{T}_{6}\right)\right)+4 E_{Z}\left(\mathcal{T}_{1}+\mathcal{T}_{2}+\mathcal{T}_{3}\right) \\
& E\left(\Delta_{6 n}^{2}\right)=E_{Z}\left(\mathcal{T}_{6}\right)+2 E_{Z}\left(\mathcal{T}_{1}+\mathcal{T}_{2}+\mathcal{T}_{3}\right)
\end{aligned}
$$

where $\mathcal{T}_{1}, \mathcal{T}_{2}, \mathcal{T}_{3}, \mathcal{T}_{5}$, and $\mathcal{T}_{6}$ are as defined in equations (34)-(36) and (39)-(40). Moreover, note that Lemma A5 and equations (41)-(43) imply that $K_{n}^{-2} E\left(\Delta_{5 n}^{2}\right) \rightarrow 0$ and $K_{n}^{-2} E\left(\Delta_{6 n}^{2}\right) \rightarrow 0$ as $n \rightarrow \infty$, which, in turn, imply that $K_{n}^{-2}\left|E\left(\Delta_{5 n} \Delta_{6 n}\right)\right| \rightarrow 0$ by applying the Jensen and the Cauchy Schwarz inequalities. The desired result then follows immediately.

Lemma A12: Under Assumptions 1-6, suppose that $r_{n} \rightarrow \infty$ as $n \rightarrow \infty$ such that $\frac{\sqrt{K_{n}}}{r_{n}} \rightarrow 0$. Then, the following statements are true: 
(a) let

$$
\bar{\Psi}_{n}=\frac{C_{n}^{\prime} Z_{n}^{\prime} Z_{n} C_{n}}{b_{n}^{2} r_{n}}
$$

then $\bar{\Psi}_{n}=O_{\text {a.s. }}(1)$; moreover, there exists a positive integer such that is positive definite almost surely.

(b) $\frac{C_{n}^{\prime} Z_{n}^{\prime \prime}\left[P_{Z_{n}} H_{1 n}-H_{2 n}\right] V_{n}}{b_{n} r_{n}} \stackrel{p}{\rightarrow} 0$ as $n \rightarrow \infty$;

(c) $\frac{C_{n}^{\prime} Z_{n}^{\prime} V_{n}}{b_{n} r_{n}} \stackrel{p}{\rightarrow} 0$ as $n \rightarrow \infty$;

(d) $\frac{V_{n}^{\prime}\left[P_{Z_{n}} H_{1 n}-H_{2 n}\right] V_{n}}{r_{n}} \stackrel{p}{\rightarrow} 0$ as $n \rightarrow \infty$.

\section{Proof of Lemma A12:}

Part (a) can be proved using arguments similar to that given for the proof of part (a) of Lemma A1 in Chao and Swanson (2002). Parts (b)-(d) can be shown by tedious but straightforward mean square calculation; see the proofs of parts (c), (f), and (g) of Lemma A1 in Chao and Swanson (2002) for similar arguments. For the sake of brevity, we omit the proofs here.

\section{Appendix B}

This part of the appendix contains proofs of the main theorems of this paper.

\section{Proof of Theorem 3.1:}

We shall only prove part (b) since part (a) can be shown by standard arguments whereas proof of part (c) is similar to that of part (b) but involving only the bilinear component and not the linear component. To prove part (b), note that for $n$ sufficiently large, so that $\left(Z_{n}^{\prime} Z_{n}\right)^{-1}$ is well-defined with probability one, we can write

$$
\begin{aligned}
\frac{Y_{2 n}^{\prime}\left[P_{Z_{n}} H_{1 n}-H_{2 n}\right] Y_{2 n}}{r_{n}}= & \frac{C_{n}^{\prime} Z_{n}^{\prime} Z_{n} C_{n}}{b_{n}^{2} r_{n}}+\frac{V_{n}^{\prime}\left[P_{Z_{n}} H_{1 n}-H_{2 n}\right] Z_{n} C_{n}}{b_{n} r_{n}} \\
& +\frac{C_{n}^{\prime} Z_{n}^{\prime} V_{n}}{b_{n} r_{n}}+\frac{V_{n}^{\prime}\left[P_{Z_{n}} H_{1 n}-H_{2 n}\right] V_{n}}{r_{n}} \\
= & \Psi_{n}+o_{p}(1),
\end{aligned}
$$


in light of Lemma A12. Thus, for $n$ sufficiently large, we can write

$$
\begin{aligned}
\left(\Psi_{n}^{-1} \Xi_{n} \Psi_{n}^{-1}\right)^{-\frac{1}{2}}\left(\widehat{\beta}_{J I V E 1}-\beta_{0}\right)= & \left(\bar{\Psi}_{n}^{-1} \Xi_{n} \bar{\Psi}_{n}^{-1}\right)^{-\frac{1}{2}} \bar{\Psi}_{n}^{-1}\left(Y_{2 n}^{\prime}\left[P_{Z_{n}} H_{1 n}-H_{2 n}\right] u_{n}\right)\left(1+o_{p}(1)\right) \\
= & \left(\bar{\Psi}_{n}^{-1} \Xi_{n} \bar{\Psi}_{n}^{-1}\right)^{-\frac{1}{2}} \bar{\Psi}_{n}^{-1}\left(b_{n}^{-1} C_{n} Z_{n}^{\prime} u_{n}\right. \\
& \left.+V_{n}^{\prime}\left[P_{Z_{n}} H_{1 n}-H_{2 n}\right] u_{n}\right)\left(1+o_{p}(1)\right)
\end{aligned}
$$

Now, consider any vector $\gamma \in R^{d}$ such that $\|\gamma\|=1$. Note that to establish the asymptotic normality of $\left(\Psi_{n}^{-1} \Xi_{n} \Psi_{n}^{-1}\right)^{-\frac{1}{2}}\left(\widehat{\beta}_{J I V E 1}-\beta_{0}\right)$, it suffices to show the asymptotic normality of

$$
\begin{aligned}
\gamma^{\prime}\left(\Psi_{n}^{-1} \Xi_{n} \Psi_{n}^{-1}\right)^{-\frac{1}{2}}\left(\widehat{\beta}_{J I V E 1}-\beta_{0}\right)= & \gamma^{\prime}\left(\bar{\Psi}_{n}^{-1} \Xi_{n} \bar{\Psi}_{n}^{-1}\right)^{-\frac{1}{2}} \bar{\Psi}_{n}^{-1}\left(b_{n}^{-1} C_{n} Z_{n}^{\prime} u_{n}\right. \\
& \left.+V_{n}^{\prime}\left[P_{Z_{n}} H_{1 n}-H_{2 n}\right] u_{n}\right) \\
= & K_{n}^{-\frac{1}{2}} \gamma^{\prime}\left(\bar{\Psi}_{n}^{-1} \bar{\Xi}_{n} \bar{\Psi}_{n}^{-1}\right)^{-\frac{1}{2}} \bar{\Psi}_{n}^{-1}\left(b_{n}^{-1} C_{n} Z_{n}^{\prime} u_{n}\right. \\
& \left.+V_{n}^{\prime}\left[P_{Z_{n}} H_{1 n}-H_{2 n}\right] u_{n}\right) \\
= & \frac{f_{n}^{\prime} u_{n}}{\sqrt{K_{n}}}+\frac{\eta_{n}^{\prime} G_{n} u_{n}}{\sqrt{K_{n}}} \\
= & \frac{W_{n}}{\sqrt{K_{n}}}
\end{aligned}
$$

where $f_{n}=b_{n}^{-1} Z_{n} C_{n} \bar{\Psi}_{n}^{-1}\left(\bar{\Psi}_{n}^{-1} \bar{\Xi}_{n} \bar{\Psi}_{n}^{-1}\right)^{-\frac{1}{2}} \gamma, \eta_{n}=V_{n} \bar{\Psi}_{n}^{-1}\left(\bar{\Psi}_{n}^{-1} \bar{\Xi}_{n} \bar{\Psi}_{n}^{-1}\right)^{-\frac{1}{2}} \gamma$, and $G_{n}=P_{Z_{n}} H_{1 n}-$ $H_{2 n}$. It follows from Lemma A11 that

$$
\gamma^{\prime}\left(\Psi_{n}^{-1} \Xi_{n} \Psi_{n}^{-1}\right)^{-\frac{1}{2}}\left(\widehat{\beta}_{J I V E 1}-\beta_{0}\right) \stackrel{d}{\rightarrow} N(0,1) \text { as } n \rightarrow \infty .
$$

The desired result follows immediately from applying the Cramer-Wold device (see Proposition 5.1 on page 114 of White, 2001).

\section{Proof of Theorem 3.2:}

Theorem 3.2 can be proved in a manner similar to Theorem 3.1. However, to do so, Lemmas A4A12 must be reformulated in terms of the corresponding quantities for $J I V E 2$, i.e., $G_{n}^{*}=P_{Z_{n}}-H_{3 n}$,

$$
\begin{aligned}
f_{n}^{*}=b_{n}^{-1}\left[I_{n}-\right. & \left.H_{3 n}\right] Z_{n} C_{n} \bar{\Psi}_{n}^{*-1}\left(\bar{\Psi}_{n}^{*-1} \bar{\Xi}_{n}^{*} \bar{\Psi}_{n}^{*-1}\right)^{-\frac{1}{2}} \gamma, \bar{\Psi}_{n}^{*}=\frac{C_{n}^{\prime} Z_{n}^{\prime}\left[I_{n}-H_{3 n}\right] Z_{n} C_{n}}{r_{n} b_{n}^{2}}, \text { and } \\
\bar{\Xi}_{n}^{*}= & \frac{1}{K_{n}} \sum_{j=1}^{n} b_{n}^{-2}\left(1-p_{j j, n}^{Z}\right)^{2} C_{n}^{\prime} Z_{n, i} Z_{n, i}^{\prime} C_{n} \sigma_{u u, j}\left(Z_{n}\right) \\
& +\frac{1}{K_{n}} \sum_{1 \leq i<j \leq n}\left[\left(g_{i j, n}^{*}\right)^{2} \Sigma_{V V, i}\left(Z_{n}\right) \sigma_{u u, j}\left(Z_{n}\right)+g_{i j, n}^{*} g_{j i, n}^{*} \sigma_{V u, i}\left(Z_{n}\right) \sigma_{V u, j}^{\prime}\left(Z_{n}\right)\right. \\
& \left.++g_{i j, n}^{*} g_{j i, n}^{*} \sigma_{V u, j}\left(Z_{n}\right) \sigma_{V u, i}^{\prime}\left(Z_{n}\right)+\left(g_{j i, n}^{*}\right)^{2} \Sigma_{V V, j}\left(Z_{n}\right) \sigma_{u u, i}\left(Z_{n}\right)\right] .
\end{aligned}
$$


Establishing Lemmas A4-A12 for $J I V E 2$ is actually notationally easier since $G_{n}^{*}=P_{Z_{n}}-H_{3 n}$ is a symmetric matrix, whereas $G_{n}=P_{Z_{n}} H_{1 n}-H_{2 n}$ is not. To avoid redundancy, however, we will not provide proofs for the case of JIVE2.

\section{Proof of Corollary 3.4:}

The desired result follows immediately given Theorem 3.1, Assumption 7, and the continous mapping theorem.

\section{Proof of Corollary 3.5:}

The desired result follows immediately given Theorem 3.2, Assumption 7', and the continous mapping theorem. 


\section{References}

Angrist, J.D., G.W.Imbens, and A.Krueger (1999), "Jackknife Instrumental Variables Estimation," Journal of Applied Econometrics, 14, 57-67.

Angrist, J. D. and A. B. Krueger (1995). "Split-Sample Instrumental Variables Estimates of the Return to Schooling," Journal of Business and Economic Statistics, 13, 225-235.

Bekker, P.A. (1994). "Alternative Approximations to the Distributions of Instrumental Variable Estimators," Econometrica, 62, 657-681.

Blomquist, S. and M. Dahlberg (1999). "Small Sample Properties of LIML and Jackknife IV Estimators: Experiments with Weak Instruments," Journal of Applied Econometrics 14, 6988.

Chao, J.C. and N.R. Swanson (2002). "Consistent Estimation with a Large Number of Weak Instruments," Working Paper, Rutgers University.

Chao, J.C. and N.R.Swanson (2003). "Asymptotic Normality of Single-Equation Estimators for the Case with a Large Number of Weak Instruments," forthcoming in Frontiers of Analysis and Applied Research: Essays in Honor of Peter C. B. Phillips, Cambridge University Press.

Donald, S.G. and W.K.Newey (2001). "Choosing the Number of Instruments," Econometrica, 69, 1161-1191.

Gänsler, P. and W. Stute (1977). Wahrscheinlichkeitstheorie (Springer-Verlag, New York).

Gao, C. and K. Lahiri (2000). A Comparison of Some Recent Bayesian and Classical Procedures for Simultaneous Equation Models with Weak Instruments, Working Paper, SUNY Albany.

Hahn, J. (2002). "Optimal Inference with Many Instruments," Econometric Theory, 18, 140-168.

Hahn, J., J. Hausman, and G. Kuersteiner (2001). "Higher Order MSE of Jackknife 2SLS," Working Paper, MIT.

Hahn, J., and J. Hausman (2002). “,” Econometrica, 70, 163-190.

Han, C. and P.C.B. Phillips (2003). "GMM With Many Moment Conditions," Working Paper, Yale University. 
Horn, R. A. and C. R. Johnson (1985). Matrix Analysis (Cambridge University Press, New York).

Kelejian, H.H. and I.R.Prucha (2001). "On the Asymptotic Distribution of the Moran I Test Statistic with Applications," Journal of Econometrics, 104, 219-57.

Kleibergen, F. (2002). "Pivotal Statistics for Testing Structural Parameters in Instrumental Variables Regression," Econometrica, 70, 1781-1803.

Magnus, J.R. and H. Neudecker (1988). Matrix Differential Calculus with Applications in Statistics and Econometrics (John Wiley \& Sons, New York).

Moreira, M. (2003). “A Conditional Likelihood Ratio Test for Structural Model," Econometrica, $71,1027-1048$.

Morimune, K. (1983). "Approximate Distributions of k-Class Estimators When the Degree of Overidentifiability Is Large Compared with the Sample Size," Econometrica, 51, 821-841.

Newey, W.K. and R. J. Smith (2003). "Higher Order Properties of GMM and Generalized Empirical Likelihood Estimators," Econometrica, forthcoming.

Phillips, G.D.A. and C. Hale (1977). "The Bias of Instrumental Variable Estimators of Simultaneous Equation Systems," International Economic Review, 18, 219-228.

Phillips, P.C.B. (1983). "Small Sample Distribution Theory in Econometric Models of Simultaneous Equations," in Z. Griliches and M.D.Intriligator, eds., Handbook of Econometrics, Vol I (North Holland, Amsterdam).

Rothenberg, T.J. (1983). "Asymptotic Properties of Some Estimators in Structural Models," in S. Karlin, T. Amemiya, and L. Goodman, eds., Studies in Econometrics, Time Series, and Multivariate Statistics, Academic Press.

Staiger, D. and J.H. Stock (1997). "Instrumental Variables Regression with Weak Instruments." Econometrica, 65, 557-586.

Smith, R. J. (1997). "Alternative Semi-Parametric Likelihood Approaches to Generalized Methods of Moments Estimation," Economic Journal, 107, 503-519. 
Stock, J.H. and M. Yogo (2002). "Testing for Weak Instruments in Linear IV Regression," Working Paper, Harvard University.

Stock, J.H. and M. Yogo (2003). "Asymptotic Distributions of Instrumental Variables Statistics with Many Weak Instruments," Working Paper, Harvard University.

Wang, J. and E. Zivot (1998). "Inference on Structural Parameters in Instrumental Variables Regressions with Weak Instruments," Econometrica, 66, 1389-1404.

White, H. (2001). Asymptotic Theory for Econometricians (Academic Press, San Diego). 